Portland State University

PDXScholar

Fall 12-13-2016

\title{
Formulation of Colloidal Suspensions of 3-mercaptopropionic acid capped PbS Quantum Dots as Solution Processable QD "Inks" for Optoelectronic Applications
}

Chase Collier Reinhart

Portland State University

Follow this and additional works at: https://pdxscholar.library.pdx.edu/open_access_etds

Part of the Chemistry Commons

Let us know how access to this document benefits you.

\section{Recommended Citation}

Reinhart, Chase Collier, "Formulation of Colloidal Suspensions of 3-mercaptopropionic acid capped PbS Quantum Dots as Solution Processable QD "Inks" for Optoelectronic Applications" (2016). Dissertations and Theses. Paper 3289.

https://doi.org/10.15760/etd.3280

This Dissertation is brought to you for free and open access. It has been accepted for inclusion in Dissertations and Theses by an authorized administrator of PDXScholar. Please contact us if we can make this document more accessible: pdxscholar@pdx.edu. 
Formulation of Colloidal Suspensions of 3-mercaptopropionic acid capped PbS Quantum Dots as Solution Processable QD “Inks" for Optoelectronic Applications

by

Chase Collier Reinhart

A dissertation submitted in partial fulfillment of the requirements for the degree of

Doctor of Philosophy

in

Chemistry

Dissertation Committee:

Erik Johansson, Chair

Dean Atkinson

Andrea Goforth

Mark Woods

Raj Solanki

Portland State University

2016 
(C) 2016 Chase Collier Reinhart 


\begin{abstract}
The use of colloidal quantum dots (QDs) for photovoltaic energy conversion is a nascent field that has been dominated for well over a decade by the use of 3-mercaptopropionic acid (3-MPA) capped PbS QDs. These QDs are routinely deposited via an in situ solid state ligand exchange process that displaces the native oleate ligand on the $\mathrm{PbS} \mathrm{QD}$ surface. This ligand exchange procedure is wasteful of material and has been demonstrated to leave numerous impurities that limit electronic performance of the asdeposited QD devices. Until the last few years there was very little understanding in chemical literature as to many important aspects of QD chemistry for this material pairing outside the framework of a QD solid. In this work, a colloidal suspension of 3MPA capped PbS QDs in DMSO was formulated and investigated to probe ligand dynamics and optical properties of the suspended colloid. QD bound 3-MPA was found to be in dynamic exchange with "free" ligand in solution by ${ }^{1} \mathrm{H}-\mathrm{NMR}$ spectroscopy. Optical properties and colloidal stability were found to be heavily dependent on the presence of a significant excess of free ligand. $\mathrm{PbS}$ QDs were also found to be highly photo-catalytic towards oxidative dimerization of 3-MPA to its dimer, dithiodipropionic $\operatorname{acid}(\mathrm{dTdPA})$.
\end{abstract}

After an initial colloidal suspension was achieved, attempts were made to directly deposit the colloid as a QD “ink" to form optoelectronic devices. While photo-switchable devices were obtained, ultimately it was determined that DMSO was a largely incompatible solvent choice for solution processing methodologies. Subsequently, 3MPA capped PbS QD colloids were obtained in volatile organic solvents amenable to 
solution processing by the addition of a stabilizing ammonium salt. These QD colloids maintained excellently resolved optical properties and were able to form conformal coatings from simple evaporative deposition. The ligand chemistry of this colloid was extensively investigated via NMR and optical spectroscopy. These QDs were also found to be highly photo-catalytic towards conversion of monomer 3-MPA to dTdPA. 


\title{
Dedication
}

\author{
With all my love...
}

To Paul, Deborah, Juanita, and Garrett, I do not hope that I might make you proud so much as I might continue to bring you love and happiness as you do for me.

To Sara, I cannot begin to describe the sublime texture with which you enrich my every waking moment.

I am humbled by you.

Blessed is he who learns how to engage in inquiry, with no impulse to harm his countrymen or to pursue wrongful actions, but perceives the order of immortal and ageless nature, how it is structured.

- $\quad$ Euripides, Fragment from an Unnamed Play

The whole world? The whole universe? Overhead, obscurity unveiled a star. One tremulous arrow of light, projected how many thousands of years ago, now stung my nerves with vision, and my heart with fear. For in such a universe as this what significance could there be in our fortuitous, our frail, our evanescent community?

But now irrationally I was seized with a strange worship, not, surely of the star, that mere furnace which mere distance falsely sanctified, but of something other, which the dire contrast of the star and us signified to the heart. Yet what, what could thus be signified? Intellect, peering beyond the star, discovered no Star Maker, but only darkness; no Love, no Power even, but only Nothing. And yet the heart praised.

- $\quad$ Olaf Stapledon, Star Maker 


\section{Acknowledgments}

To Dr. Johansson, thank you for both your presence and, when appropriate, absence as a guiding hand during my investigations. I will always remember your uncaged excitement-“Milliamps, Chase! Milliamps!” —upon completion of my first prototype device. I will also remember the sobering two years of follow-up work that eventually ended that project much less dramatically. Subsequently, I greatly appreciate the freedom to define and pursue my own spectroscopic studies even as I initially struggled to confirm their utility with useful and interpretable data. Quantum dots in suspension, it turns out, are about as hard to control as we initially worried.

To the research group, including the small army of undergraduates I mentored, thank you for your input and encouragement over the years.

To my committee, thank you for your continuing patience and collaboration in navigating this process.

To my friends, thank you for enduring me in my excitement and despair. Thank you for helping me remember where the ground is, why I began, and how to see value in myself.

To Natasja Swartz and Rob Jensen, I cannot begin to express any proper measure of gratitude for your friendship. I would not have made it through these years without the bouyancy you lent me.

To Anthony Mason, Daniel Shaw, and Kate Gavrin, thank you for keeping me in your heart all these years across short and long miles. 


\section{Table of Contents}

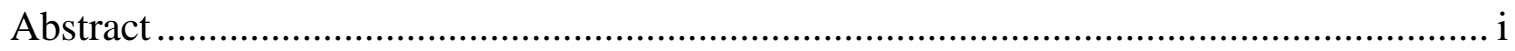

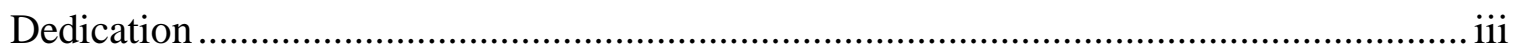

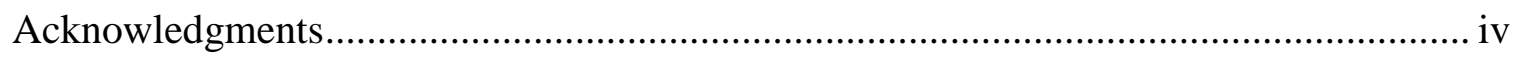

List of Tables .................................................................................................... vii

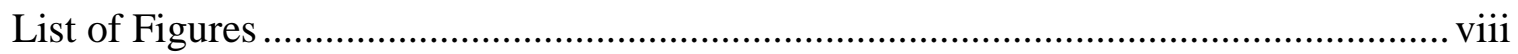

Glossary and List of Abbreviations/Symbols ..................................................... xiv

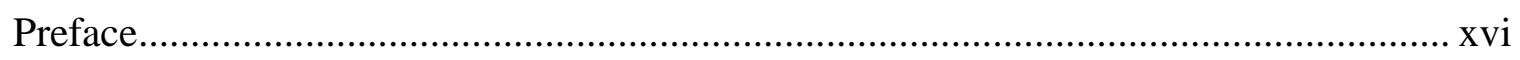

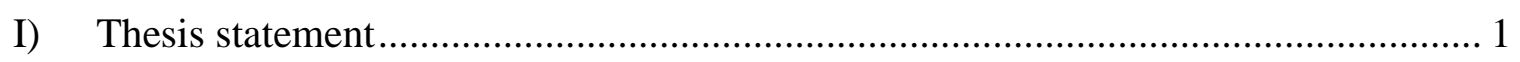

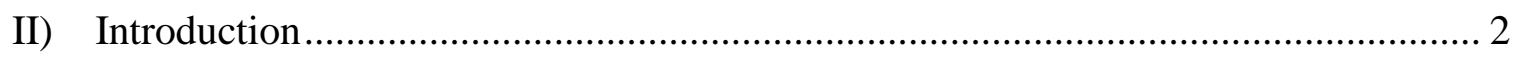

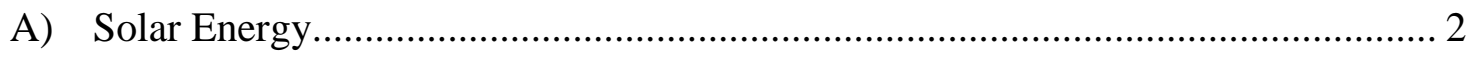

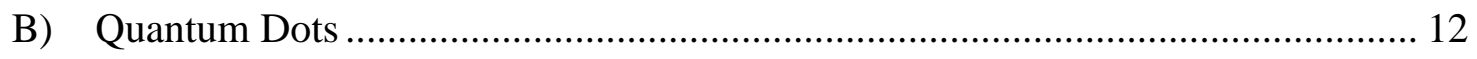

III) Colloidally Prepared 3-Mercaptopropionic Acid Capped Lead Sulfide Quantum

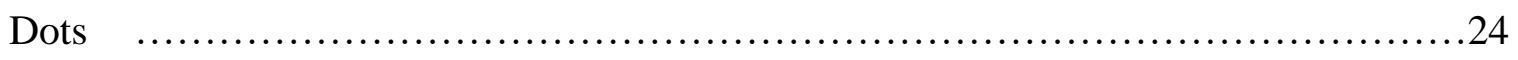

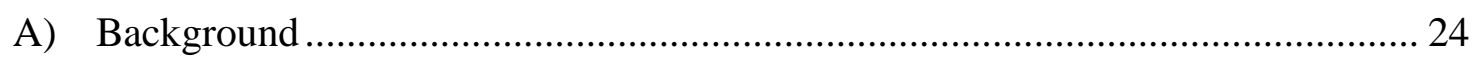

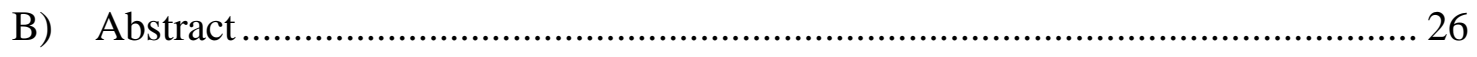

C) Experimental Methods .............................................................................. 26

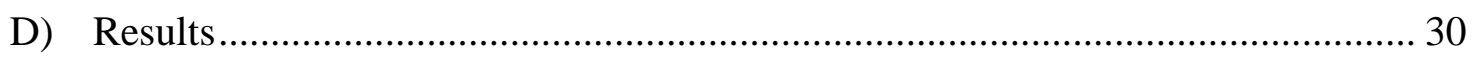

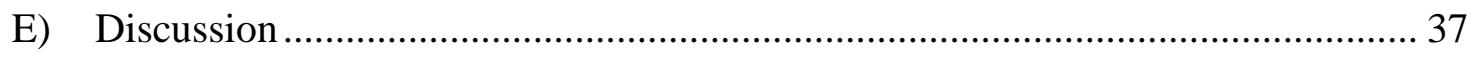

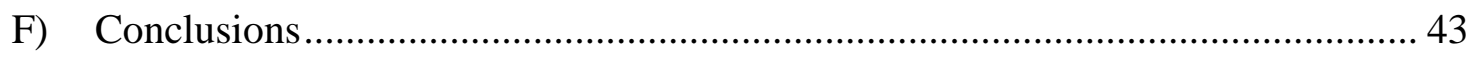

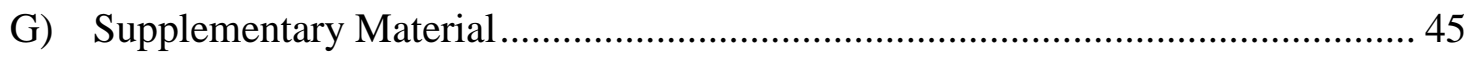


IV) Colloidal 3-mercaptopropionic acid Capped Lead Sulfide Quantum Dots in a Low

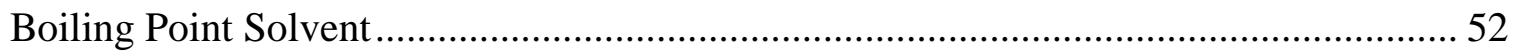
A) Background 52

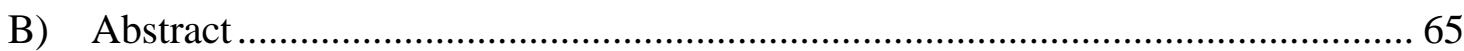

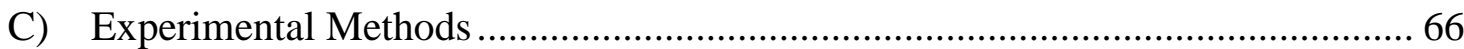

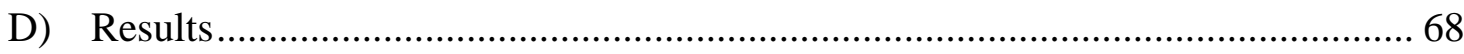

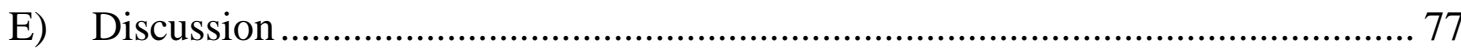

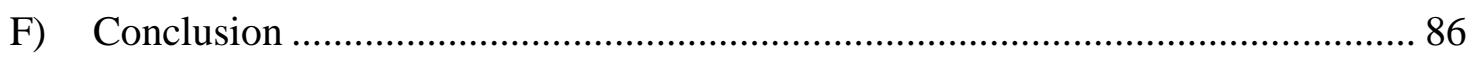

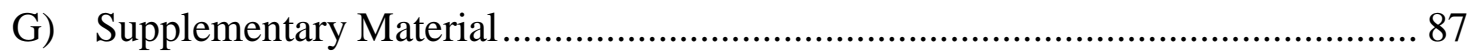

V) Conclusions and Future Directions .............................................................. 102

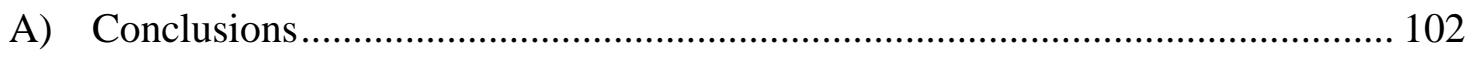

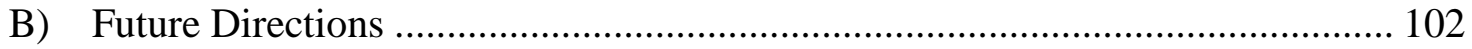

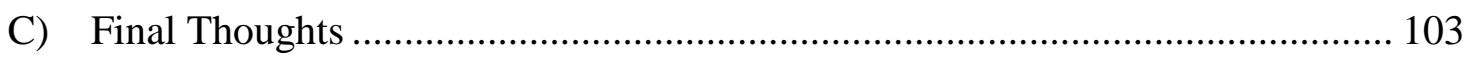

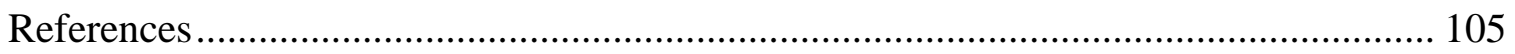




\section{List of Tables}

Table III-1. Peak fit data from emission experiments................................................ 31 


\section{List of Figures}

Scheme II-1. Combustion reaction of a simple hydrocarbon releasing $\mathrm{CO}_{2}, \ldots \ldots \ldots \ldots \ldots \ldots . . . . . .2$

Figure II-1. Cartoon demonstrating the simplified processes of A) photoexcitation and B)

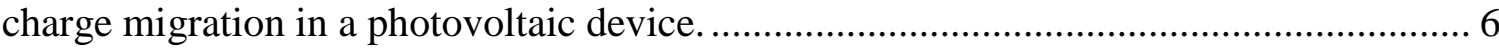

Figure II-2. Evolution of semiconductor band structure ............................................. 7

Figure II-3. Cartoon demonstrating the formation of a charge directing field gradient. .... 9

Figure II-4. Chronologically ordered champion research PV cells as tracked by the

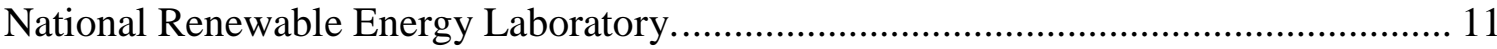

Figure II-5. Physical representation of nanoscale crystallites. .................................... 13

Figure II-6. A) Typical absorption (solid) and emission (dotted) spectrum of a PbS QD sample.

Chart II-1. Oleic acid and oleylamine are common ligands for QDs that act as colloidal stabilizers bound to the QD surface.

Figure II-7. Terrestrial solar insolation spectrum with overlapping absorption profiles for differently sized PbS QD samples. 17

Chart II-2. 3-mercaptopropionic acid and other common ligands for cross-linking QDs 18

Figure II-8. Sequential deposition of PbS QD solids from a colloidal suspension 19

Chart III-1. Terephthalic acid, a bifunctional crosslinking candidate ligand for fabricating $\mathrm{PbS}$ QD thin films. 
Figure III-1. Absorption (solid traces) and luminescence (dotted traces) spectra of colloidal 3-MPA exchanged PbS QDs in 10\% v/v 3-MPA in DMSO $(2.5 \mathrm{mg} / \mathrm{mL})$ and oleate capped $\mathrm{PbS}$ QDs in toluene.

Figure III-2. ${ }^{1} \mathrm{H}-\mathrm{NMR}$ spectra of 3-MPA with and without the presence of PbS QDs. ... 32

Figure III-3. ${ }^{1} \mathrm{H}-\mathrm{NMR}$ spectra of oleyl species under varying experimental conditions indicating interaction, or lack thereof, with $\mathrm{PbS}$ QDs.

Figure III-4. Absorption (solid trace) and emission (dotted trace) spectra of 3-MPA capped $\mathrm{PbS}$ QDs at different PbS QD concentrations. 34

Figure III-5. Absorption (A) and luminescence (B) spectra of 3-MPA capped PbS QDs at $2.5 \mathrm{mg} / \mathrm{mL}$ across a seven day period of aging under both dark and light conditions stored in an ambient environment. 35

Scheme III-1. Oxidative dimerization of 3-MPA to dTdPA........................................ 36

Figure III-6. 1H-NMR spectra of 3-MPA exchanged QDs in DMSO-D 6 under different aging conditions.

Figure S III-1. NMR spectra for full assignment of 3-MPA in DMSO-D 6.

Figure S III-2. ${ }^{1} \mathrm{H}-\mathrm{NMR}$ of $\mathrm{OaH}(\mathrm{A})$ and $\mathrm{Oa}-\mathrm{PbS}$ QDs (B) in $\mathrm{CDCl} 3$ with assignments labeled relative to the displayed structure of oleic acid. 46

Figure S III-3. ${ }^{1} \mathrm{H}-\mathrm{NMR}$ Spectra in DMSO-D 6 showing identification of dTdPA growth during aging.

Figure S III-4. (A) ${ }^{1} \mathrm{H}-\mathrm{NMR}$ spectra of 3-MPA in DMSO-D 6 during aging and (B) calculated concentrations of 3-MPA and dTdPA. 48 
Figure S III-5. ${ }^{1} \mathrm{H}-\mathrm{NMR}$ spectra of 3-MPA aging in DMSO.

Figure S III-6. ${ }^{1} \mathrm{H}-\mathrm{NMR}$ spectra of 3-MPA exchanged QDs in DMSO-D 6 50

Figure S III-7. Dynamic light scattering of freshly prepared (three overlapping solid lines) and $24 \mathrm{hr}$ aged (dotted lines) samples of 3-MPA QDs in 10\% v/v 3-MPA in DMSO-D6 at different QD concentrations. 51

Figure IV-3. SEM micrographs of 3-MPA capped PbS QD films on $\mathrm{TiO}_{2}$ substrates..... 55

Figure IV-4. Cyclic voltammetry and open circuit potential of a directly deposited 3MPA capped PbS QD photovoltaic device. 57

Figure IV-5. Cyclic voltammetry and open circuit potential of an in situ deposited 3-MPA capped PbS QD photovoltaic device. 58

Figure IV-6. Simultaneously plotted cyclic voltammetry and open-circuit potential from prototype and in situ films. 59 Figure IV-7. Absorption and emission spectra of OA-PbS QDs after exposure to solutions of secBA and 3-MPA in $\mathrm{CHCl}_{3}$ 60

Chart IV-1. Sec-butylamine and didodecyldimethylammonium bromide (dDDdMABr), two of the stabilizing additives used to suspend 3-MPA capped PbS QDs in volatile organic solvents. 61

Figure IV-8. Rotational correlation time, $\tau c$, vs radius via the Stokes-Einstein relation for small spherical objects. 64

Figure IV-9. Projected 2-dimensional spectra showing differentiation of free $v s$ bound ligand signal signing in NOESY type experiments. 65 
Figure IV-10. Absorption (solid) and emission (dashed) spectra of Oa-PbS QDs in $\mathrm{CHCl} 3$ and $\mathrm{Oa}-\mathrm{PbS}$ QDs resuspended in various organic solvents with 3-MPA and dDDdMABr present in a 20:20:1 ratio of [3-MPA]:[dDDdMABr]:[Oa-]

Figure IV-11. A) Representative absorption (solid) and emission (dashed) spectra from Oa-PbS QDs resuspended in solutions of equimolar amounts of 3-MPA and dDDdMABr in $\mathrm{CHCl}_{3}$ 70

Chart IV-2. Oleic acid (OaH), 3-mercaptopropionic acid (3-MPA), and didodecyldimethylammonium bromide (dDDdMABr). Each molecule's distinguishable ${ }^{1} \mathrm{H}$ environments are labeled. 71

Figure IV-12. ${ }^{1} \mathrm{H}$ NMR spectra in $\mathrm{CDCl}_{3}$ of 3-MPA and dDDdMABr resonances. ........ 72

Figure IV-13. ${ }^{1} \mathrm{H}$ NMR spectra of oleyl resonances. 73

Figure IV-14. NOESY spectra demonstrating ligand interaction with PbS QDs. 74

Chart IV-3. Dithiodipropionic acid (dTdPA) with distinguishable proton environments labeled. 75

Figure IV-15. ${ }^{1} \mathrm{H}$ NMR spectra and quantification of dTdPA formation from colloids of Oa-PbS QDs with 3-MPA/dDDdMABr added in a 20:20:1 ratio of [3MPA]:[dDDdMABr]:[Oa $\left.{ }^{-}\right]$in $\mathrm{CDCl}_{3}$ 75

Figure S IV-1. Absorption (solid) and emission (dotted) spectra of Oa-PbS QDs demonstrating excitonic preservation upon addition of a stabilizing additive. 88

Figure S IV-2. NOESY spectra of oleyl species with and without QDs present. 89 
Figure S IV-3. NOESY spectrum of an exchanged QD colloid with a 20:20:1 mixture of [3-MPA]:[dDDdMABr]:[Oa $\left.{ }^{-}\right]$. 90

Figure S IV-4. ${ }^{1} \mathrm{H}-\mathrm{NMR}$ spectra of Oa-PbS QDs with 3-MPA/dDDdMABr added in a 20:20:1 ratio of [3-MPA]:[dDDdMABr]:[$\left[\mathrm{Oa}^{-}\right]$. 91

Figure S IV-5. ${ }^{1} \mathrm{H}$ NMR spectra of 3-MPA/dDDdMABr solutions in a 1:1 ratio in $\mathrm{CDCl}_{3}$.

Figure S IV-6. Photograph of Oa-PbS QDs with 3-MPA/dDDdMABr added in a 20:20:1 ratio of [3-MPA]:[dDDdMABr]:[Oa $\left.{ }^{-}\right]$after $\sim 48$ hours of aging. 93

Figure S IV-7. ${ }^{1} \mathrm{H}$ NMR spectra in $\mathrm{D}_{6}$-DMSO of (blue) precipitated solid from aging experiments in $\mathrm{CDCl}_{3}$ and (red) a similar solution spiked with a small grain of commercially obtained dTdPA. 94

Figure S IV-8. Absorption (solid) and emission (dashed) spectra of Oa-PbS QDs with 3MPA/dDDdMABr added in a 20:20:1 ratio of [3-MPA]:[dDDdMABr]:[Oa $\left.{ }^{-}\right]$. 95 Chart IV-4. Didodecylmethylamine (dDDMA) and dodecylamine (DDA). Each molecule's distinguishable ${ }^{1} \mathrm{H}$ environments are labeled. 96 Figure S IV-9. Expanded ${ }^{1} \mathrm{H}$ NMR spectra of oleyl resonances under varying exchange conditions. 96 Figure S IV-10. ${ }^{1} \mathrm{H}-\mathrm{NMR}$ in $\mathrm{CDCl}_{3}$ of expanded oleyl species focusing on carboxylic acid resonances.

Figure S IV-11. Expanded ${ }^{1} \mathrm{H}$ NMR spectra of oleyl resonances upon addition of amines. 
Figure S IV-12. NOESY spectrum of A) a solution of dDDdMABr in $\mathrm{CDCl}_{3}$ and $\mathrm{B}$ ) an OA-PbS QD colloid in $\mathrm{CDCl}_{3}$ with dDDdMABr added to solution in a 20:1 [dDDdMABr]:[Oa $\left.{ }^{-}\right]$ratio.

Figure S IV-13. Absorption (solid) and emission (dashed) spectra of Oa-PbS QDs upon exposure to 3-MPA and sec-butylamine in $\mathrm{CHCl}_{3}$. 101 


\section{Glossary and List of Abbreviations/Symbols}

3-MPA: 3-mercaptopropionic acid, a short chain bi-functional ligand employed in $\mathrm{PbS}$ quantum dot optoelectronics.

CDCl3: Chloroform, with each proton replaced by a deuterium nucleus.

Colloid: A suspension of non-soluble material in a liquid.

COSY: Correlation spectroscopy, a two-dimensional NMR technique that transfers magnetization between coupled nuclei.

Cross-linking: Molecular coordination of two or more quantum dots with a bi-functional ligand coordinating each nanoparticle through separate coordinate bonds.

Cross-peak: An off main-diagonal peak in two-dimensional NMR spectroscopy indicating transfer of magnetization.

DDA: Dodecylamine, a common quantum dot ligand.

dDDdMABr: Didodecyldimethylammonium bromide.

dDDMA: N,N-didodecylmethylamine.

DMSO-D6: Dimethylsulfoxide with each native proton replaced by a deuterium nucleus.

DOSY: Diffusion ordered spectroscopy, a pseudo-two-dimensional NMR technique that separates NMR signal by diffusion parameters.

dTdPA: Dithiodipropionic acid, a dimer of 3-mercaptopropionic acid.

Efficiency (Photovoltaic): A ratio of the power output of a device to the insolation power on the active surface of the device.

$\mathbf{E}_{\mathrm{g}}$ : Bandgap, the energy separation between the highest occupied and lowest unoccupied orbitals. 
In situ exchange: A common solid-state ligand exchange technique wherein a deposited layer of quantum dots is exposed to a solution containing a new ligand that displaces the native ligand before being washed.

Insolation: Irradiance from our sun (Sol), measured in power per unit area.

Ligand: a molecules coordinated to a metal atom. In the context of quantum dots, a ligand refers to a molecule bound to the quantum dot.

NMR: Nuclear magnetic resonance spectrscopy

NOESY: Nuclear Overhauser effect spectrscopy, a two-dimensional NMR technique that utilizes through-space transfer of magnetization.

OaH: Oleic acid, a common ligand for PbS quantum dots.

PbO: Lead (II) Oxide, a common pre-cursor for PbS quantum dot synthesis.

PbS: Lead (II) Sulfide, a common ore and semiconducting material.

Photovoltaic device: a device that transduces light energy to electrical energy

Quantum dot "Ink": A suspension of quantum dots in a solvent intended for direct deposition via solution processing.

Quantum dot: Semiconductor nanocrystallite of a size where strong quantum confinement effects are observed.

Solar energy: Energy in the form of radiation emanating from the sun.

Spin coating: A thin-film deposition technique wherein a volatile solution is dispensed onto a rotating substrate to drive even deposition of solute as the solvent evaporates.

$\mathrm{TiO}_{2}$ : Titanium (IV) dioxide, a ubiquitously employed wide-bandgap semiconductor commonly used as an acceptor material in heterojunction devices. 


\section{Preface}

This thesis is based on the work contained in the following paper, reproduced in Chapter III with the permission of the publisher:

Reprinted with permission from Reinhart, C.C.; Johansson, E., Colloidally Prepared 3Mercaptopropionic Acid Capped Lead Sulfide Quantum Dots. Chem. Mater. 2015, 27 (21), 7313-7320. Copyright 2016 American Chemical Society.

Other portions of this work have been disseminated during the following presentations:

Reinhart, C. C., Johansson, E., Colloidally Suspended 3-MPA capped PbS Quantum Dots. 250th ACS National Meeting - Boston, MA, August 2015

Reinhart, C. C., Johansson, E., Comparison of Optical and Electronic Properties of PbS Quantum Dot Films Made Using pre-exchange or in situ Exchange of Ligands. 248th ACS National Meeting - San Francisco, CA, August 2014

Reinhart, C. C., Johansson, E., Optical Properties of Lead Sulfide Quantum Dots with Modified Capping Agents for Possible Solar Energy Collection. 68th ACS Northwest Regional Meeting - Corvallis, OR, July 2013 
Reinhart, C. C., Johansson, E., Thiostannate capped PbS quantum dot sensitized metal oxides for solar-to-electrical energy conversion. 19th International Conference on Photochemical Conversion and Storage of Solar Energy - Pasadena, CA, July 2012

Chapter IV represents a manuscript in preparation. 


\section{I) THESIS STATEMENT}

3-mercaptopropionic acid (3-MPA) capped PbS quantum dots (QDs) can be prepared in stable colloidal suspensions with promise for optoelectronic device applications and as thiol oxidation catalysts.

Specifically:

1. Deliberate control over solvent, quantum dot, ligand, and co-ligands allows for the preparation of colloidally stable suspensions of 3-mercaptopropionic acid capped lead $\mathrm{PbS}$ quantum dots.

2. Their high luminescence in suspension is reflective of their effective energy preservation in champion QD photovoltaic devices.

3. 3-MPA-PbS QD colloids can be prepared in solvents amenable to solution processing of conformal films by addition of a stabilizing co-ligand.

4. Dynamic exchange on the quantum dot surface of 3-mercaptopropionic acid and its oxidation product allows light-enhanced surface catalyzed oxidation of 3mercaptopropionic acid to the corresponding dithiol dimer. 


\section{II) INTRODUCTION}

\section{A) Solar Energy}

\section{1) A Statement on Energy Motivations}

The modern global energy ecology is a complex landscape of production and consumption with one primary observation. Despite constant changes in sources and consumption of energy, there is a dominant trend: we consume more energy globally as time progresses. Worldwide energy consumption has more than doubled between 1971 and 2013, with the use of coal and natural gas also doubling in that timeframe to keep pace with demand. ${ }^{1}$ Combustible "fossil" fuels have dominated the energy landscape into the modern era.

$$
\mathrm{CH}_{4}+2 \mathrm{O}_{2} \rightarrow \mathrm{CO}_{2}+2 \mathrm{H}_{2} \mathrm{O}
$$

Scheme II-1. Combustion reaction of a simple hydrocarbon releasing $\mathrm{CO}_{2}$.

Regulation and energy efficiency improvements have led to projections that indicate the United States may stabilize $\mathrm{CO}_{2}$ emissions in the coming decades. ${ }^{2}$ Despite this, the industrially developing world is consuming massive amounts of energy, primarily using fossil fuels (in this case coal and oil) as manufacturing increases to unprecedented levels. ${ }^{3}$ While the effects of $\mathrm{CO}_{2}$ on the environment are not entertained in this document, common-sense implies a diverse energy portfolio will likely be necessary to satisfy the demands of an increasing global-population. Inherently exhaustible resources that are inhomogeneously distributed ultimately lead to an unstable energy economy. This demands that we distinguish a difference between historically used exhaustible energy sources and renewable energy sources that are ubiquitously available. 


\section{2) Solar Energy as a Renewable Energy Source}

Recent statistics put annual global energy consumption at 13594.11 million tons of oil equivalent (Mtoe). ${ }^{1}$ To put this in a more standard unit of energy, this is equivalent to $1581 \times 10^{2}$ teraWatt hours (TWh). ${ }^{\mathrm{i}}$ This puts the average rate of energy consumption at 18.05 TW. ${ }^{\text {ii }}$ Fortuitously, the sun provides a bounty of energy to the Earth's surface in the form of solar radiation. Solar radiation insolates at a rate of $120000 \mathrm{TW}$, and an oft cited statistic from 2005 states that more energy hits the Earth's surface in an hour than we consume, as a planet, in a year's time. ${ }^{4}$ Illustrating the rapid growth of energy consumption, this statistic no longer holds for 2013 ; now only $~ 76 \%$ of the energy consumed in a year is delivered to the Earth's surface via insolation from the sun. .ii $^{\text {ii }}$ Regardless, the sun still delivers an astonishing $6.649 * 10^{5} \%$ of the amount of power we demand, illustrating the massive scale of this resource. ${ }^{\text {iv }}$

\section{3) Challenges for Solar Energy}

Solar energy is massively and ubiquitously available, carbon neutral, ${ }^{\mathrm{v}}$ and capable of being captured or stored in a variety of technologies with their own unique challenges: direct solar-to-electric, solar fuel generation, and storage as biomass through photosynthesis. ${ }^{5}$ Direct energy generation works when the Sun is currently insolating an

\footnotetext{
${ }^{\text {i }} 1$ Mtoe $=1.163 * 10^{1} \mathrm{TWh} .13594 .11 \mathrm{Mtoe} * 1.163 * 10^{1} \mathrm{TWh} / \mathrm{Mtoe}=1518 * 10^{2} \mathrm{TWh}$.

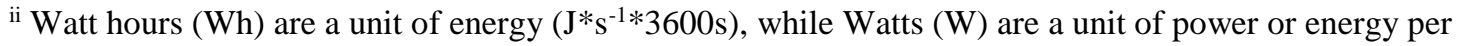
unit time $\left(\mathrm{J}^{*} \mathrm{~s}^{-1}\right)$.

iii Hourly solar insolation is $120000 \mathrm{TWh}$. Annual energy consumption as of 2013 is $151800 \mathrm{TWh}$.

iv $120000 \mathrm{TW} / 18.05 \mathrm{TW} * 100 \%=6.649 * 10^{5} \%$

${ }^{v}$ Not accounting for the carbon footprint of manufacturing means of capturing insolated energy. This is highly variant based on method of capture and rapidly shifting as photovoltaic production increases.
} 
area where there is a demand for power but not when power is needed at night. As such, it is considered a variable source of power. To address the constant need for power energy must be stored as a convertible source. Solar fuel generation (such as the splitting of water into molecular hydrogen and oxygen) requires storage of produced fuels. One of the larger challenges is the scale of infrastructure required to make photovoltaic (PV) energy conversion a viable method of producing power. Geothermal, wind, and solar energy currently represent $\sim 1.2 \%$ of global energy consumed. ${ }^{1}$ Projections indicate that the largest growth in renewables will be in solar and wind, ${ }^{2}$ both of which require large amounts of physical infrastructure with scaling. For instance, the amount of area needed to generate enough energy to power the United States using $10 \%$ efficient PV modules has been estimated to be $1.7 \%$ of its land area; though many argue a construction project of this size is not feasible, it is roughly the size of the US highway system and is certainly within our engineering capibilities. ${ }^{5}$ Additionally, this statistic utilizes relatively low efficiency PV modules, whereas crystalline silicon PV modules now exceed a confirmed $25 \%$ conversion efficiency. ${ }^{\mathrm{vi}, 6}$ Solving the problem of scaling will require large area devices with low material cost and high efficiency.

\section{4) Photovoltaic Technologies, Current and Emerging}

The public is primarily familiar with solar energy conversion in the form of silicon based PV modules. Early silicon based p-n junction photocells reached photo-conversion

${ }^{\text {vi }}$ Conversion efficiency is defined as the ratio of the output power of the module to the power incident on the module surface. The figure is often given in percentage. 
efficiencies of $\sim 6 \%$ while modern modules exceed $25 \%$ efficiency. ${ }^{6,7}$ There are numerous types of direct solar-to-electric conversion technologies that utilize different material types with varying efficiencies; for example GaAs, CuInGaSe (CIGS), and multi-junction cells have shown efficiencies as high as $46 \%{ }^{6}$ A material may be considered a viable candidate for typical photovoltaic applications as long as it satisfies some base criteria: it should be a semiconductor capable of allowing photo-excited charges to migrate and be collected.

\section{5) Principles of Photovoltaics}

The key principle of photovoltaic technology is the transduction of light energy to electrical energy. This is accomplished in a step-wise fashion. First, light energy is absorbed by a semiconductor material with a separation of electronic states. The separation between occupied and unoccupied states is referred to as the bandgap, $\mathbf{E}_{\mathbf{g}}$. Second, excited charges, i.e. electrons and electron-holes, separate by migrating to opposite ends of the device where they perform work under some external load by following a free energy gradient and are ultimately re-collected. This process is demonstrated schematically below in Figure II-1. 


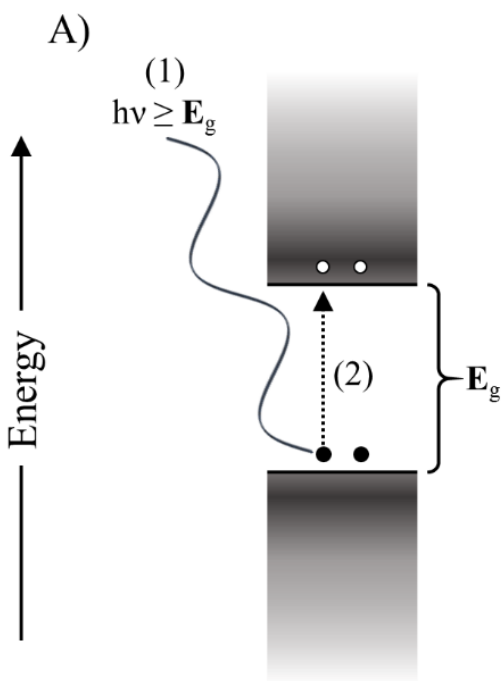

B)

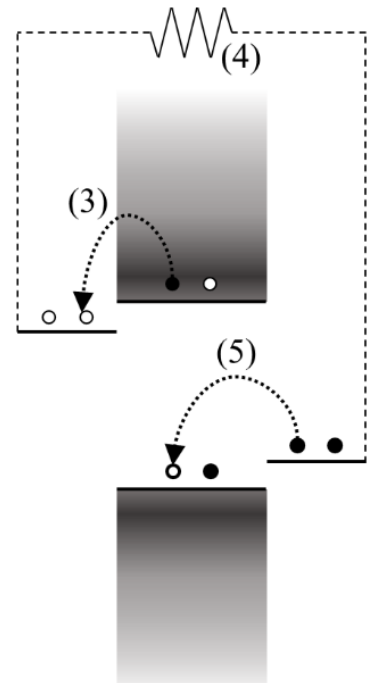

Photoexcitation

\section{Charge Migration}

Figure II-1. Cartoon demonstrating the simplified processes of A) photoexcitation and B) charge migration in a photovoltaic device. The step-wise processes include (1) absorption of a photon with energy greater than or equal to the optical bandgap energy. This results in (2) promotion of an electron to a higher energy level. Excited charges (3) flow into an acceptor material and (4) travel through an external circuit where they perform work. Finally, electrons (5) fill in unoccupied states and return to their original energy level.

\section{Absorption}

During the process of absorption, a frontier orbital electron is promoted from an occupied state to an unoccupied state at an energy difference equivalent to the energy of the absorbed photon. ${ }^{\text {vii }}$ In a solid these frontier orbitals function analogously to HOMOLUMO $^{\text {viii }}$ states in molecular orbitals. ${ }^{8}$ Due to the density of atoms in a solid there are a

\footnotetext{
vii Photon energy is defined as $\mathrm{E}=\mathrm{h} v$, where $\mathrm{E}$ is energy, $\mathrm{h}$ is Planck's constant, and $v$ is the frequency of a photon.

viii HOMO-LUMO refers to the concept of frontier orbitals in molecules. HOMO is an abbreviation for the highest occupied molecular orbital, whereas LUMO represents the lowest unoccupied molecular orbital.
} 
large number of orbitals of sufficiently proximal energy to form an effective continuum that is referred to as an energy "band". This evolution of energy bands from atomic orbitals is demonstrated schematically below in Figure II-2.

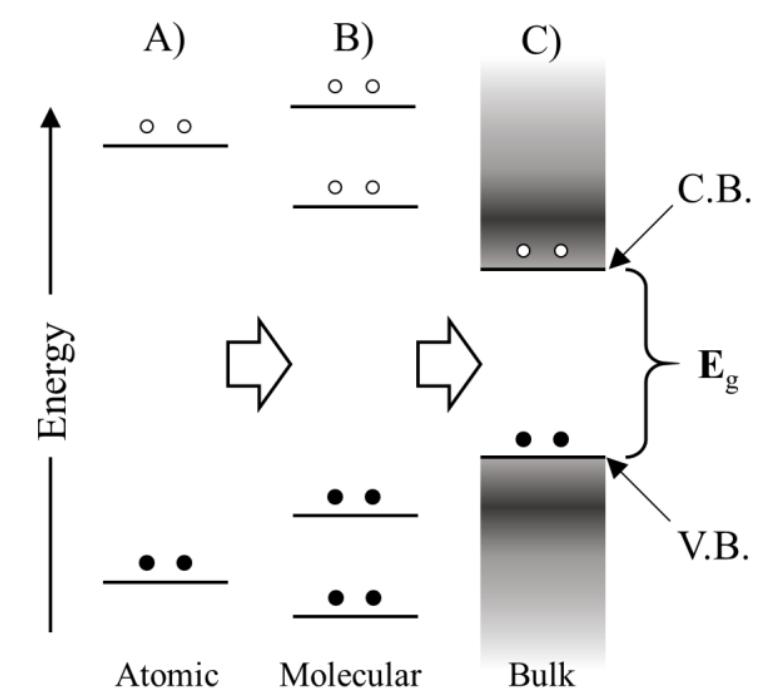

Figure II-2. Evolution of semiconductor band structure. Energy levels combine from A) atomic orbitals into distributed B) molecular orbitals and ultimately C) bulk energy bands in solids. V.B. here refers to the valence band edge, whereas C.B. is the conduction band edge. $\mathbf{E}_{\mathrm{g}}$, the optical bandgap, is equal to the difference in energy between the two band edges. Solid circles represent occupied states, while empty circles represent unoccupied states. The color gradient represents density of states.

The evolution of energy bands manifests from the linear combination of atomic orbitals across a framework where the number of atoms is extremely large. As a result, dense energy states become delocalized throughout the solid. The energy structure of these bands_-including the energy position of the frontier orbitals as well as the value of $\mathbf{E}_{\mathrm{g}}-$ can be calculated with reasonable accuracy with some disagreement between empirically derived values due to the complexity of the calculations. ${ }^{9-11}$ The highest energy occupied orbital is referred to as the valence band edge, while the lowest energy unoccupied orbital 
is referred to as the conduction band edge as shown in Figure II-2C. It is not necessary for an energy gap to exist between the frontier orbitals of an extended periodic solid. The presence and width of such an energy gap is the primary distinction between metals, semiconductors, and insulators.

\section{Charge Migration and Separation}

The dominant process for separation of charges in a photovoltaic device is charge carrier drift directed by a free energy gradient. ${ }^{9}$ In silicon-based photovoltaics this is accomplished by combining a material homo-junction of inversely doped regions to form a diode ${ }^{\mathrm{ix}}$ that separates free charge carriers. This junction forms kinetic pathways for differently signed carriers that are spatially directional and largely irreversible. This is typically described via a thought experiment that is illustrated in Figure II-3. The material boundary is referred to as the metallurgical junction; and in silicon PV these junctions are formed by diffusing dopant atoms in through opposite ends of the material as opposed to physically combining two differently doped materials. The net effect is the formation of an energy gradient by a phenomenon referred to as band bending. ${ }^{12,13}$

ix A diode is an electrical element that allows charge to flow easily in one direction while preventing it from moving in the opposite direction. 
A)

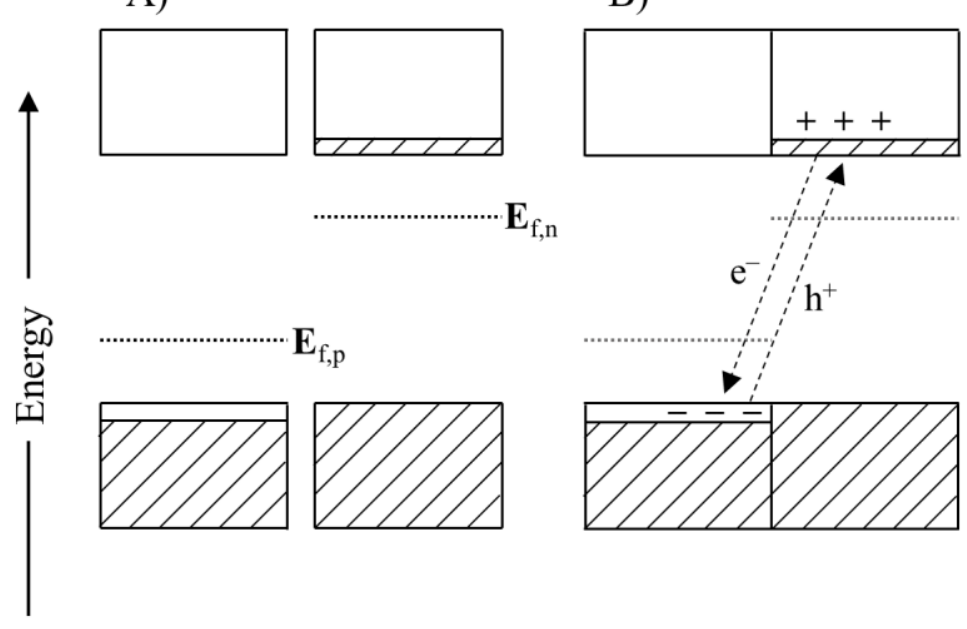

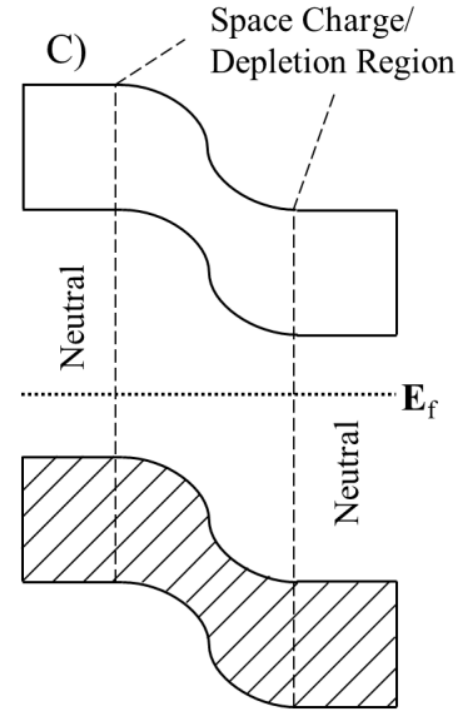

Figure II-3. Cartoon demonstrating the formation of a charge directing field gradient. In this cartoon the xdimension relates to an un-scaled physical dimension in a solid material. A) a p-doped and n-doped semiconductor that have not made contact have distinct fermi levels indicated as $\mathbf{E}_{\mathrm{f}, \mathrm{p}}$ and $\mathbf{E}_{\mathrm{f}, \mathrm{n}}$ respectively. ${ }^{\mathrm{x}}$ Upon contact between the two materials B) electrons begin flowing from the $n$-doped region into the $\mathrm{p}$ doped region due to the difference in electrochemical potential. This leaves behind positively charged dopant atoms that have been depleted of their electrons. The inverse happens in the p-doped region, where acceptor atoms acquire negative charge from the migrant electrons. After equilibrating, C) a local electric field is established in the spatial region where depletion has occurred that modifies the local positions of the band edges and forms an energy gradient. This local electric field also prevents any further charge carrier diffusion and the semiconductor is considered at equilibrium.

Any free carriers in the area under the influence of the established field - known as the depletion region - are subject to directional flow depending on their charge. Electrons

\footnotetext{
${ }^{\mathrm{x}} \mathbf{E}_{\mathrm{f}}$ refers to the fermi level of a semiconductor. The fermi level is the energy position where there is a $1 / 2$ probability of finding an electron at equilibrium; it is important to note that this value does not guarantee a state is actually present, occupied or unoccupied, at that energy position.
} 
follow the energy gradient from the p-doped region to the n-doped region while holes (electron vacancies) flow in the opposite direction. At equilibrium the diffusion of carriers from the differently doped regions is opposed by the static potential of the accumulated charges on either side. At this point the two dominant mechanisms of charge transport, carrier drift and diffusion, are equally balanced and there is no net flow of carriers.

Upon photoexcitation this equilibrium is perturbed and free carriers that successfully diffuse to the depletion region are swept across the junction due to the electric field. ${ }^{14}$ Carriers that do not successfully diffuse to the depletion zone eventually recombine with an oppositely signed carrier. Because of this, PV devices rely on effective charge transport with extensive depletion to support long diffusion distances and charge migration direction, respectively.

\section{6) Quantum Dots as Candidates for Solar Energy Conversion}

While this example of PV operation has been given for a device consisting of an equally doped p-n homo-junction, the principle of operation holds true for heterojunction devices that combine different materials. Quantum dots (QDs) are typically employed in semiconductor heterojunction-type devices with a wide bandgap metal oxide (e.g., $\mathrm{TiO}_{2}$, $\mathrm{ZnO}, \mathrm{SnO}$ ) acceptor material. The National Renewable Energy Laboratory keeps a record of device efficiencies based on material type and architecture (Figure II-4) that shows progress in different areas of PV material research. ${ }^{15}$ 


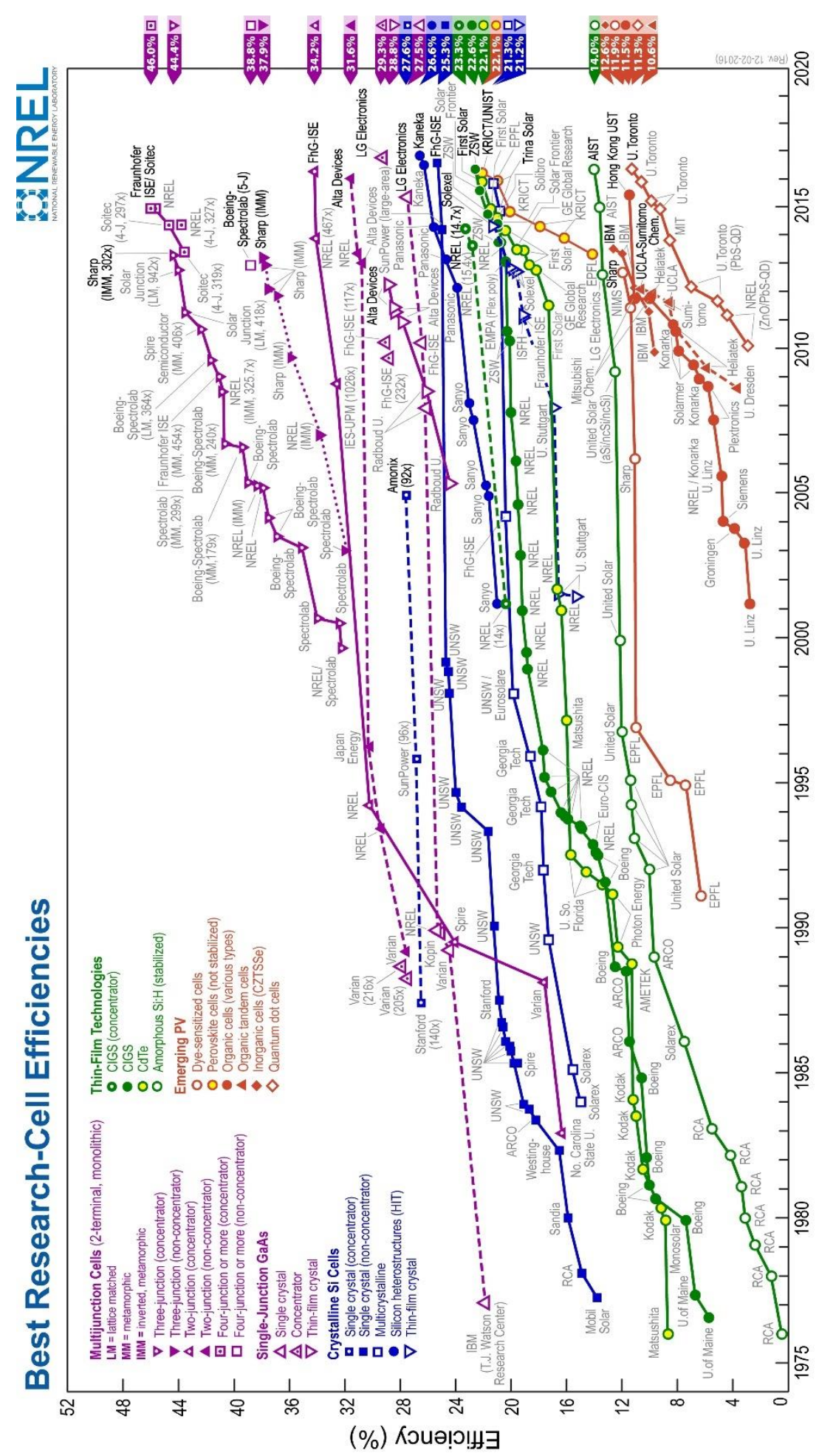

Figure II-4. Chronologically ordered champion research PV cells as tracked by the National Renewable Energy Laboratory. This plot is courtesy of the National Renewable Energy Laboratory, Golden, CO. ${ }^{15}$ 
Since the early 2000's, metal oxide semiconductors sensitized with QDs have been aggressively pursued as an alternative candidate to silicon PV. The work described in this document focuses on the fabrication and characterization of an optoelectronic material (3-mercaptopropionic acid capped PbS QDs) used in leading efficiency QD PV applications. The following section describes the physics of QDs, their use to-date in PV applications, and challenges specific to QD PV that motivated the work presented herein.

\section{B) Quantum Dots}

\section{1) Physical Description of $Q D s$}

Quantum dots are semiconductor nanocrystals of a physical dimension that is smaller than the Bohr exciton ${ }^{\mathrm{i}}$ radius for the bulk material from which they are made. ${ }^{16}$ They have size and shape dependent optical and electronic properties with an inverse correlation between nanocrystal size and bandgap that were first described in the early 1980’s. ${ }^{17}$

\footnotetext{
${ }^{\mathrm{i}}$ An exciton is a pseudo-particle formed from a coulombically bound electron and hole pair that results from excitation of a valence band electron in a semiconductor into the conduction band.
} 

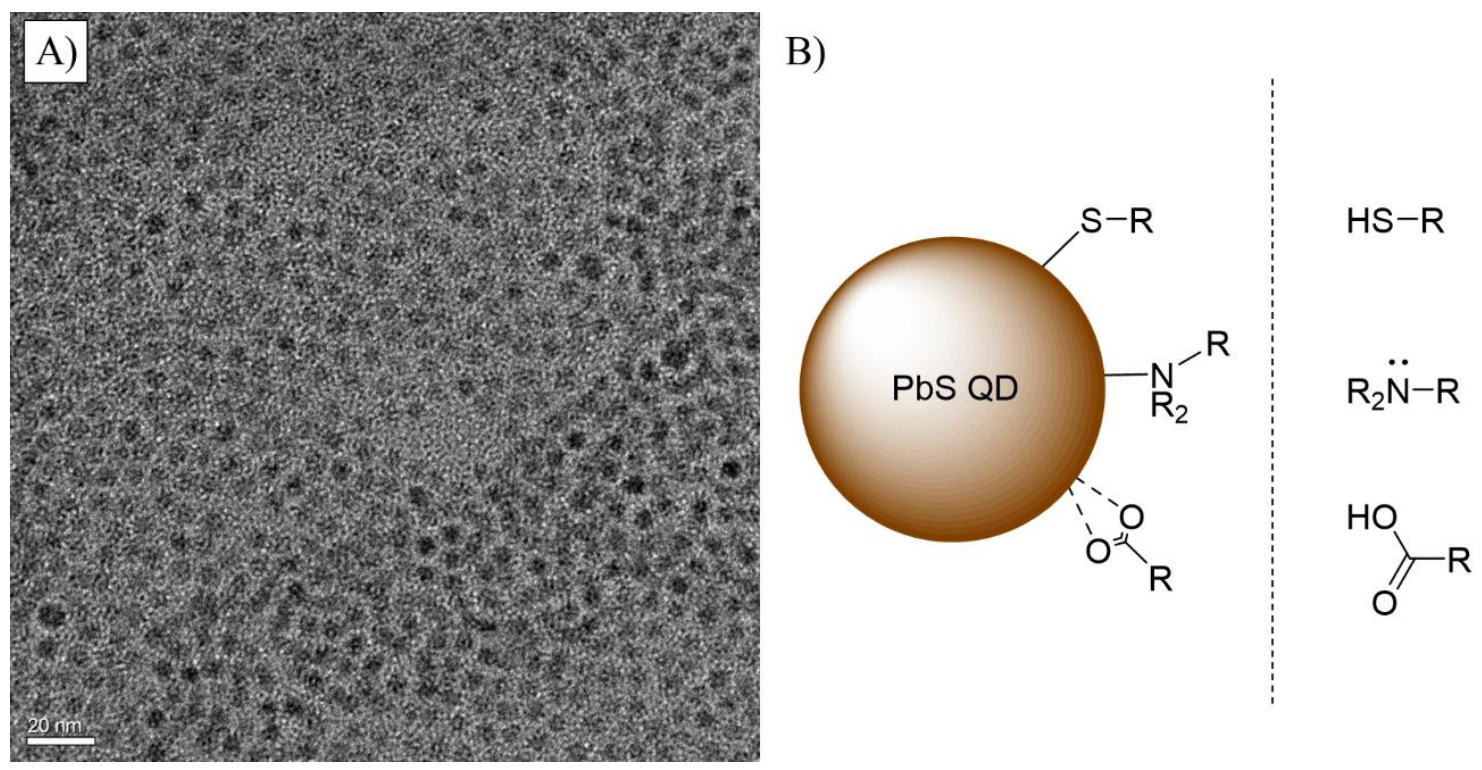

Figure II-5. Physical representation of nanoscale crystallites. A) TEM Micrograph of a sample of oleate capped PbS QDs. Scale bar is $20 \mathrm{~nm}$. B) Cartoon of a spherical QD and generalized binding schemes for common ligand types.

QDs are particles that lie between the size of bulk solids and small molecules. The size range where strong quantum confinement of excitons occurs is material dependent; ${ }^{18}$ for $\mathrm{PbS}$ QDs this corresponds to a crystallite size $<20 \mathrm{~nm} .{ }^{19}$ Very small QDs are typically modeled as spherical particles surrounded by a ligand shell typically consisting of thiol, amine, or carboxylic acid containing molecules. The degree and nature of surface coverage by ligands is oft debated and highly contextual to the QD material and ligand type. ${ }^{20-22}$ At a certain size (also material dependent) the surface energy of establishing crystalline facets begins to dominate, resulting in pseudo-faceted crystals. ${ }^{23}$ Typical $\mathrm{PbS}$ QDs can be described as truncated cuboctahedra, ${ }^{24}$ though a number of factors affect crystalline shape such as: ligand identity, pre-cursor concentration, and synthesis temperature. ${ }^{25,26}$ 
Non-ligated surfaces of QDs are highly reactive and naturally condense to minimize surface energy if contact is made, ultimately forming particulate agglomerates which are much larger than the size where quantum confinement is experienced. This undesirable process must be prevented if one wishes to synthetically tune the optoelectronic properties of QDs to operate in a certain energy regime; as such, QDs are usually considered metastable materials protected by ligands bound to surface atoms. These ligands typically govern the QDs interactions with surrounding media—colloidal stability and charge transfer being two important examples of such interactions-and loss of these ligands can result in particle aggregation that destroys the unique properties of the QDs.

\section{2) Optical Properties of $Q D s$}

QDs feature discrete quantized optical transitions that are analogous to atomic absorption modes as opposed to the broadband absorption of bulk semiconductors. As QD suspensions are always a population with various sizes and shapes, idealized discrete absorption lines observed in atomic optical transitions present instead as slightly broadened peaks. ${ }^{19}$ A typical absorption and emission spectrum from a suspension of $\mathrm{PbS}$ QDs is shown in Figure II-6. 

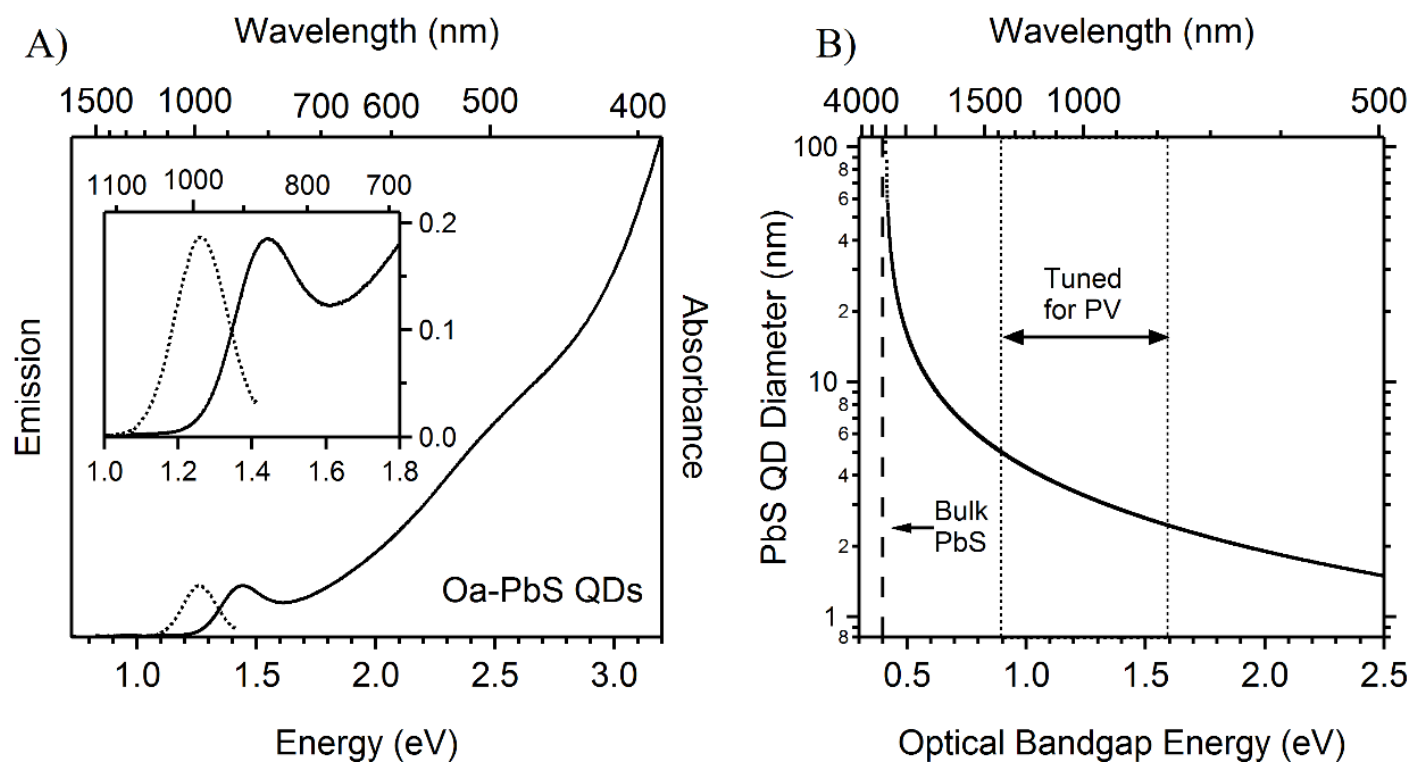

Figure II-6. A) Typical absorption (solid) and emission (dotted) spectrum of a PbS QD sample. The large absorption peak at $\sim 1.45 \mathrm{eV}$ is the $1^{\text {st }}$ excitonic absorption at the optical bandgap energy. Another (more broad) peak that represents the $2^{\text {nd }}$ excitonic transition can be seen at $\sim 2.45 \mathrm{eV}$. B) Modeled optical bandgap energy of spherical PbS QDs by particle size. ${ }^{27}$ The bulk bandgap energy of $\mathrm{PbS}(\sim 0.4 \mathrm{eV})$ is shown as a dashed line. Commonly used PbS QDs tuned for PV applications lie within the $0.9-1.6 \mathrm{eV}$ range.

Apart from the well documented size and shape dependence, QDs present a complicated chemical landscape with many parameters that can be further modified to tune their optical properties. Loss of ligands due to chemical changes at the surface can cause particle aggregation that may manifest as scattering artifacts in absorption and emission spectra. To a lesser extent, partial aggregation can also result in broadened peaks and slow loss of resolved excitonic transitions. Solvato- and thermochromic effects are being established, though the physical origin of the observed changes is often subject to debate. ${ }^{28-31}$ The nature of the dielectric environment surrounding QDs can also lead to red-shifting of both absorption and emission modes. ${ }^{32}$ Ligand identity and surface 
coverage affect optical properties as well, ${ }^{33,34}$ with multiple studies demonstrating ligand concentration influences the surface structure of QDs. ${ }^{35-37}$ Ligand identity has also been shown to cause structural deformations at the surface of small QDs that can modify their optical properties. ${ }^{26}$ These observed effects on QD optical properties are each their own active field of interest and rely on optical spectroscopy as a complicated but useful diagnostic tool for probing targeted properties of QDs.

\section{3) PbS QDs for use in Solar Energy by Photoconversion}

Colloidally stable PbS QDs with well-defined optical properties have been reported since 2003. ${ }^{38}$ Since then, colloidal PbS QDs capped with long aliphatic ligands such as oleic acid or oleylamine) are routinely prepared in narrow size dispersions ranging from 3 to $13 \mathrm{~nm}$, and show high photoluminescence quantum yields. ${ }^{39-42}$

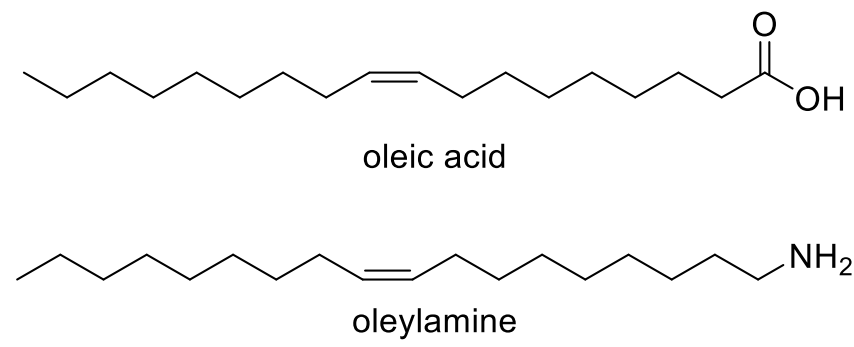

Chart II-1. Oleic acid and oleylamine are common ligands for QDs that act as colloidal stabilizers bound to the QD surface.

$\mathrm{PbS}$ QDs are uniquely strong absorbers, ${ }^{27,43}$ which makes them a good candidate for thin film PV applications. They have long lived excited states, ${ }^{27,44}$ a key parameter for maintaining excited charge transfer through QD films operating in the diffusion regime. They also have a relatively large excitonic Bohr radius of approximately $20 \mathrm{~nm}$ that allows a wide range of energy tuning for a variety of spectral windows. ${ }^{19}$ Figure II-7 
demonstrates the ability to tune QD absorption spectra for a desired source using the solar insolation spectrum as an example.

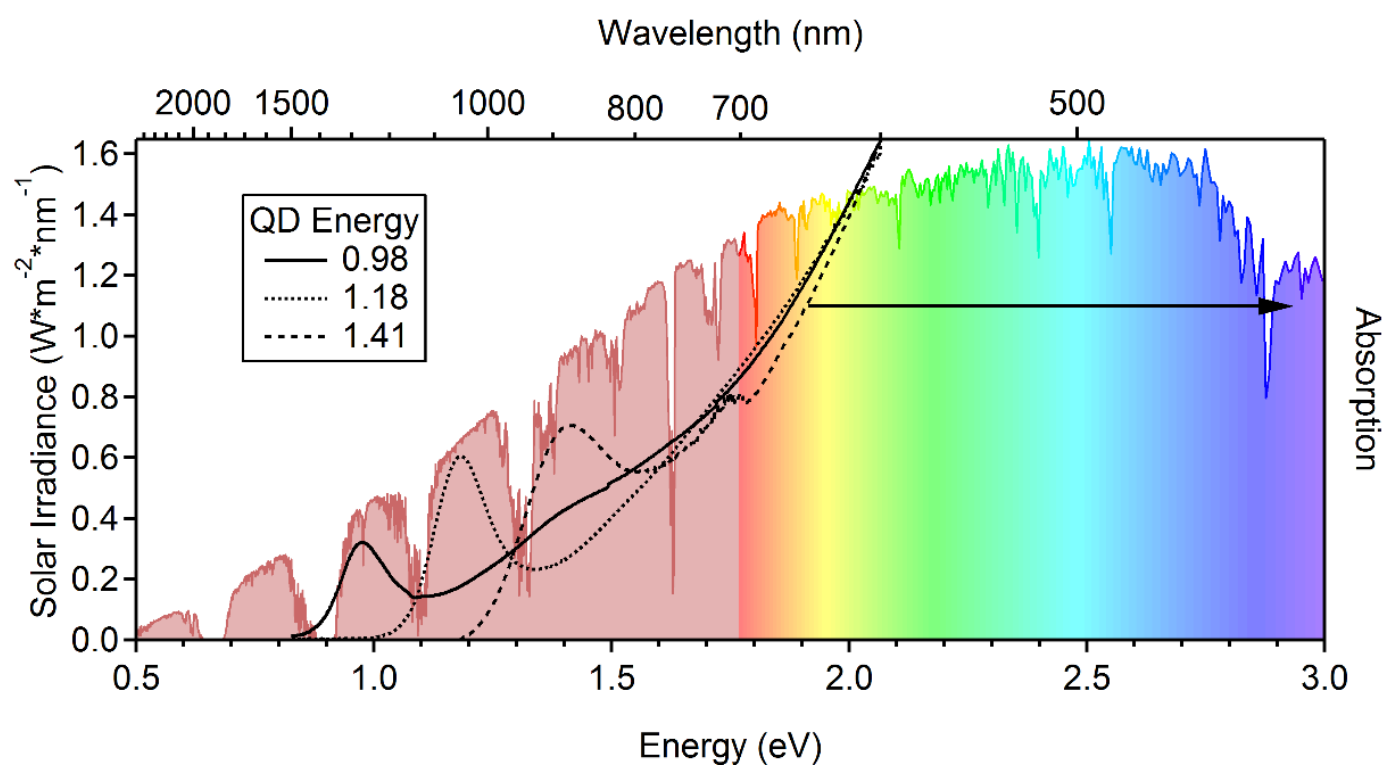

Figure II-7. Terrestrial solar insolation spectrum with overlapping absorption profiles for differently sized PbS QD samples.

Tuning of absorption bands allows for maximal overlap of absorption and emission profiles which means capture of more incident power for PV applications. This also means that energy levels of the QD are modified relative to any heterojunction acceptor materials that can affect charge transfer by modifying kinetic driving force for carriers navigating across materials. ${ }^{45-47}$

\section{4) PbS QD Solids for PV Applications}

A new class of PbS QD solid based photovoltaics using cross-linked PbS QDs was first reported in 2005 and quickly attracted significant interest. ${ }^{48-50}$ Shown in Chart II-2, 3mercaptopropionic acid (3-MPA) was first used in 2010 to prepare PbS QD solid photovoltaics with a reported energy conversion efficiency of $5.1 \% .{ }^{45}$ Recently, 
photovoltaic devices made with colloidal PbS QD solid absorbers have demonstrated efficiencies up to $10.8 \% .^{51}$

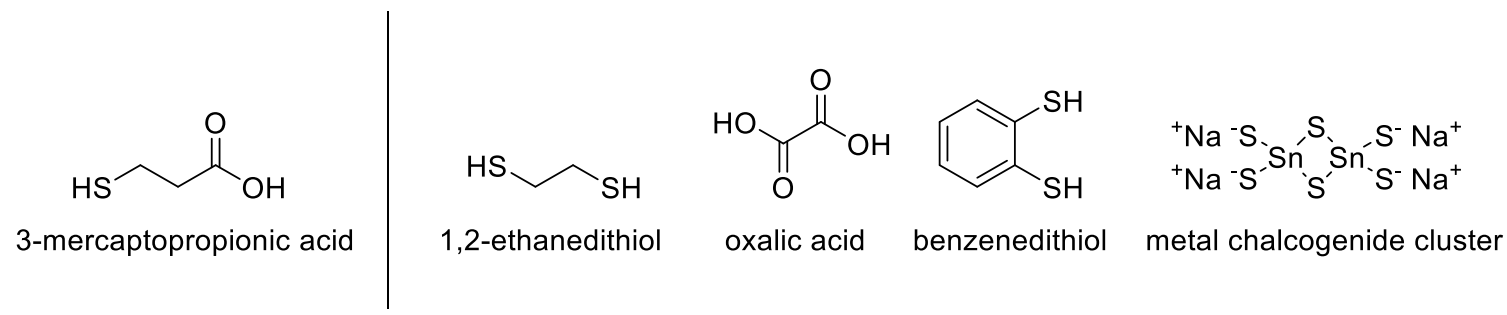

Chart II-2. 3-mercaptopropionic acid and other common ligands for cross-linking QDs

Absorbers made from colloidal PbS QDs and 3-MPA linkers continue to be among the best photovoltaic devices of this class. ${ }^{52-63}$ Numerous short ligands such as 1,2ethanedithiol, oxalic acid, benzenedithiols, and thiostannates, also shown in Chart II-2, have been used to cross link lead chalcogenide QDs to form QD solids. ${ }^{64-68}$ However, 3MPA still remains an attractive ligand because its use results in low densities of mid-gap states allowing for collection of charge carriers over relatively long distances outside the depletion region. ${ }^{69}$

\section{Film Formation}

Typically, 3-MPA linked PbS QD solids for optoelectronic applications are prepared using in situ ligand exchange procedures. ${ }^{45,52,54,56,60-63,66,70-76} \mathrm{PbS}$ QDs capped with long, electrically insulating ligands are deposited on a semiconducting substrate and ligand exchange is carried out on the as-deposited solid with 3-MPA containing solutions. Following this, metallic $(\mathrm{Au})$ films are deposited on top of the QD film and on the substrate itself to allow electrical contacting. This process is demonstrated schematically in Figure II-8. 
1) Spin coat oleate capped $\mathrm{PbS}$ QD film
2) Treat film with 3-MPA solution in $\mathrm{MeOH}$

3) Wash film with fresh $\mathrm{MeOH}$

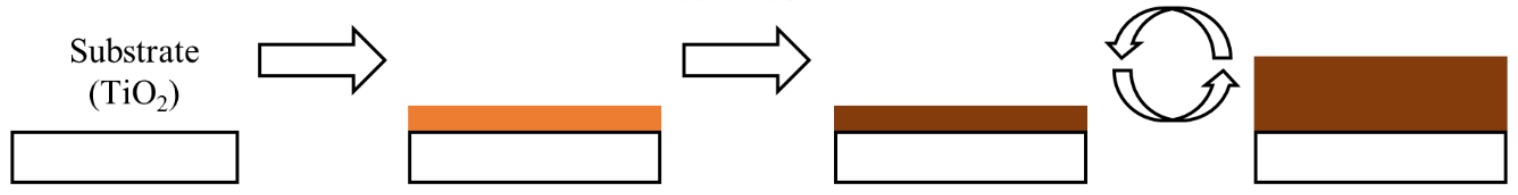

Figure II-8. Sequential deposition of PbS QD solids from a colloidal suspension. The process begins with 1) spin coating PbS QDs to form a solid array followed by 2) in situ ligand exchange to replace oleate with 3-MPA before 3) washing displaced oleate out of the film. This process is 4) repeated until the QD array has reached the desired thickness.

The product is an electrically conductive semiconductor solid comprised of 3-MPA cross-linked PbS QDs.

\section{Consequences of In Situ Exchange}

Though control over 3-MPA capped PbS QD film deposition has seen recent advancements in application such as the transition to spray deposition, ${ }^{58}$ it has been demonstrated that the in situ exchange process inherently leaves complex reaction products as impurities in the exchanged films which have been shown to negatively affect the optoelectronic properties of synthesized QD solids. ${ }^{60,77}$ QD solid homogeneity is significantly affected by both ligand type and experimental parameters during ligand exchange, ${ }^{78}$ and sequential spin-coating deposition of QD solids utilizes only 1-10\% of the total amount of PbS QDs used to form a solid. ${ }^{59}$ The solvent typically used for in situ exchange has also been shown to negatively affect mid-gap state densities. ${ }^{60}$ Furthermore, in situ deposition protocols provide little opportunity to tune 3-MPA/QD 
interactions. The formulation of QD "inks" instead may ameliorate these unsatisfactory limitations of QD solids.

\section{5) $Q D$ "Inks"}

Just as solids are periodic atomistic assemblies, QD solid arrays are ideally well organized periodic assemblies. Control over inter-QD organization is highly desired for optoelectronic applications and has been the focus of numerous studies and reviews. ${ }^{79-81}$ The formulation of QD “inks" for this purpose has been explored with some success. ${ }^{53,59,82-88}$ In such systems a native capping agent (typically a long chain organic surfactant) is exchanged with a new ligand while maintaining the suspended QDs in a volatile solvent. The goal is to obtain a colloid of QDs having the desired capping agent that can be deposited directly using solution processing techniques and without postdepositional modification. In addition to leaving optoelectronically deleterious byproducts,${ }^{60,77}$ post-depositional modification of QD solids routinely results in film cracking due to volume contraction in the ligand layer during ligand exchange that can disrupt inter-QD connectivity. ${ }^{78,89,90}$

Particle assembly using solvent based techniques have shown to be a useful approach for building nanocrystal "superlattices" that may circumvent the effects associated with post-depositional ligand modification. ${ }^{91,92}$ Film formation with PbS QD inks has frequently been shown to be dependent on ligand type and coverage. ${ }^{42,93,94}$ Direct deposition of $\mathrm{PbS} \mathrm{QD}$ solids for photovoltaic applications has been achieved previously, ${ }^{59,82}$ including one recent report of centrifugal casting of 3-MPA-capped QDs. ${ }^{53}$ 
"Inks" are a semantic distinction differentiable from any other suspension by virtue of their intended application for deposition as formulated. Generally, the desired characteristics of a well-characterized and stable QD suspension for optoelectronic applications are: narrow and well defined optical absorption and emission peaks, high photoluminescence yield, narrow size polydispersity, high colloidal stability, and a wellcharacterized ligand shell.

\section{Prior Preparations of 3-MPA Capped PbS QDs}

Some preparations of 3-MPA capped PbS quantum dots through ligand exchange or direct synthetic routes have been reported previously. For example: 3-MPA capped PbS QDs from 2.5-9.9 $\mathrm{nm}$ have been prepared via aqueous ligand exchange by adding dried oleic acid capped QDs to a basic solution of concentrated 3-MPA ( 8.3 M 3-MPA, pH adjusted to 11 with tetramethylammonium hydroxide). ${ }^{95}$ The QDs maintained excellent optoelectronic properties after deposition onto $\mathrm{TiO}_{2}$ single crystal substrates as evidenced by the subsequent multiple exciton collection studies but were not stable in suspension. The QDs were reported to agglomerate rapidly which prevented analysis of optical properties or ligand chemistry.

3-MPA capped PbS QDs have also been prepared via aqueous synthesis. ${ }^{96}$ These QDs were prepared by reacting lead acetate with a basic solution $(\mathrm{pH}=9)$ of 3-MPA before injecting a solution of $\mathrm{Na}_{2} \mathrm{~S}$ and heating to grow QDs to different sizes; the resulting colloids had unstable luminescence and their absorption spectra were not obtained. These QDs were synthesized using an acetate containing precursor that is a known ligand for QD surfaces and no attempt at analyzing the ligand identity on the surface of the QDs 
was made. Similarly, 3-MPA-capped PbSe QDs have been prepared by aqueous synthesis but did not have their optical properties or ligand chemistry characterized. ${ }^{97}$

3-MPA capped PbS QDs have furthermore been prepared by ligand functionalization of pulsed laser deposited PbS QDs, ${ }^{98}$ and 3-MPA has been used to link PbS QDs to metal

oxide surfaces. ${ }^{99}$ In both of these studies 3-MPA was exposed to the QDs after deposition on various substrates without preparing a colloidal suspension. In all of the aforementioned cases the resulting 3-MPA capped PbS QDs either did not achieve colloidal stability or demonstrate 3-MPA was the actual capping agent.

\section{Towards "Stable" Colloidal Suspensions of 3-MPA capped PbS QDs} Very recently, centrifugal casting of colloidal 3-MPA capped PbS QDs from a DMSO suspension was reported with detailed analysis of the resulting 3-MPA QD solids. ${ }^{53}$ The focus of this report was on the optoelectronic and structural properties of the PbS QD solid. In this work, a suspension of 3-MPA capped PbS QDs was centrifugally deposited onto a substrate loaded into a centrifuge tube; centrifugal isolation of QDs is typically only possible when suspended QDs are partially aggregated. The as deposited QD solids were evaluated as the active material in photovoltaic devices wherein up to $6.1 \%$ solar conversion efficiency was achieved with a single step deposition process.

Separately, we concurrently reported the preparation and characterization of colloidal suspensions of 3-MPA capped PbS QDs in DMSO with a focus on the optical properties and ligand shell chemistry. ${ }^{100}$ Oleic acid bound to PbS QDs (Oa-PbS QDs) was replaced by 3-MPA in dimethyl sulfoxide. It was shown that PbS QDs in dimethyl sulfoxide were 
stabilized through dynamic binding to 3-MPA when it was present in significant excess. This work is covered in Chapter III.

Ultimately, DMSO is not compatible with QD deposition techniques that rely on solvent evaporation (e.g., spin- or dip-coating) due to its low volatility. Furthermore, DMSO is also a weak oxidizing agent, capable of oxidizing thiols such as 3-MPA to the corresponding dithiols. ${ }^{86,101,102}$ Ideally, QD “ink" solvents are nonreactive and amenable to direct solution deposition through spin-coating, evaporative deposition, etc. However, in light of the prominent role of 3-MPA linking of PbS QD solids for optoelectronic applications there are still surprisingly few reports focused on the preparation of colloidally stable 3-MPA capped PbS QDs in solvents that are more amenable for solution processing. The formulation of 3-MPA capped PbS QD colloids in solution processable solvents is covered in Chapter IV. 


\section{III) COLLOIDALLY PREPARED 3-MERCAPTOPROPIONIC ACID CAPPED LEAD SULFIDE QUANTUM DOTS}

\section{A) Background}

The material in this chapter is adapted with permission from: Reinhart, C.C.; Johansson, E., Colloidally Prepared 3-Mercaptopropionic Acid Capped Lead Sulfide Quantum Dots. Chem. Mater. 2015, 27 (21), 7313-7320.

\section{1) Delamination of PbS QDs films during in situ exchange}

The objectives of this study were to formulate and characterize a suspension of 3-MPA capped PbS QDs to study their optical properties and ligand chemistry as a suspension. Initially, while exploring in situ ligand exchange of Oa-PbS QDs with an alternate ligand, terephthalic acid, it was observed that under the proper solvent and ligand conditions the PbS QD film would delaminate from the substrate during spin coating instead of crosslinking and leaving an exchanged PbS QD film. Terephthalic acid has very low solubility in its protonated state in all solvents other than DMSO.

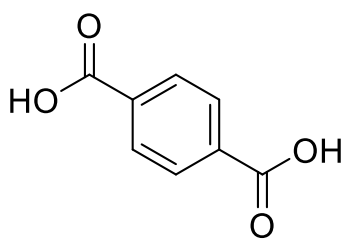

terephthalic acid

Chart III-1. Terephthalic acid, a bifunctional crosslinking candidate ligand for fabricating PbS QD thin films.

Using this phenomenon with DMSO as a solvent, a colloid of 3-MPA capped PbS QDs in DMSO was obtained. Initially, a dry film of Oa-PbS QDs was exposed to a solution of 3- 
MPA in DMSO and gently stirred until the QDs re-suspended. After isolating a narrow concentration window of [3-MPA] that produced stable colloid that was suitable for analysis this technique was modified into a bi-phasic transfer using DMSO/hexane. After ligand exchange and extraction of the QDs into the DMSO phase the colloid was analyzed via optical and NMR spectroscopy.

\section{2) NMR Spectroscopy for analyzing ligands in QD suspensions}

The surfaces of QDs are a complex and dynamic chemical environment that dictate many aspects of their stability. ${ }^{90}$ Solution NMR has emerged as a very useful tool for understanding not only what molecules associate with the surface of QDs but the manner in which they do so. ${ }^{103}$ NMR spectroscopy offers a unique advantage for analyzing ligands in QD colloids in situ. Other analysis techniques such as infrared spectroscopy (FTIR) and X-ray photoelectron spectroscopy typically require deposition onto a substrate that perturbs delicate chemical equilibria of the ligand-QD interactions in a suspension. Furthermore, NMR spectroscopy presents a unique opportunity to nondestructively probe complex nanocrystal suspensions with spatial resolution at the organic-inorganic interface. These low intensity surface signals would normally be masked by the aggregate sample when using techniques such as FTIR. NMR spectroscopy has been used with QD colloids to: quantify ligand surface density, ${ }^{20,104}$ distinguish between free and bound ligands, ${ }^{104-107}$ observe dynamic chemical exchange, ${ }^{108,109}$ and probe the inorganic surface structure of QDs. ${ }^{110}$ 


\section{B) Abstract}

Colloidally suspended PbS quantum dots stabilized with 3-mercaptopropionic acid (3MPA) were prepared via solution ligand exchange. The optical properties of the as prepared colloidal QDs were investigated by absorption and emission spectroscopy. The 3-MPA exchanged QDs luminesced with intensities comparable to oleate capped PbS QDs. The nanocrystal ligand chemistry was investigated by solution $1 \mathrm{H}$ Nuclear Magnetic Resonance (NMR) spectroscopy, which indicated a dynamic ligand shell undergoing rapid exchange. Optical properties of the QD colloid evolved over time and were correlated to the oxidative conversion of 3-MPA into dithiodipropionic acid (dTdPA) in the presence of dimethylsulfoxide (DMSO) observed by NMR.

\section{C) Experimental Methods}

\section{1) Materials}

Oleic acid (OaH) (Technical Grade, Sigma, 112-80-1), 1-octadecene (Technical Grade, Sigma, 112-88-9), bis(trimethylsilyl)sulfide (95\%, Sigma, 3385-94-2), lead oxide (99.999\%, Sigma, 1317-36-8), anhydrous toluene (Alfa Aesar, 110-54-3), anhydrous nhexane (Alfa Aesar, 110-54-3), 3,3'-dithiodipropionic acid (dTdPA) (99\%, Sigma, 111962-6), anhydrous dimethylsulfoxide (EMD, 67-68-5), dimethylsulfoxide-D 6 (DMSO-D 6 , 99.9\% D, Sigma, 2206-27-1), 1,4-dichlorobenzene ( $\geq 99 \%$, Sigma, 106-46-7). All materials were used as received unless otherwise noted. 


\section{2) PbS Quantum Dot Synthesis}

Oleate capped $\mathrm{PbS}$ quantum dots (Oa-PbS QDs) were prepared by following a modified procedure from literature. ${ }^{74}$ Briefly, oleic acid and 1-octadecene were heated separately under vacuum to $90{ }^{\circ} \mathrm{C}$ for 24 hours before being transferred into a glovebox (MBraun Labstar) containing less than $0.5 \mathrm{ppm} \mathrm{H}_{2} \mathrm{O}$ and $\mathrm{O}_{2}$. Oleic acid (9.6 mmol, $\left.3.057 \mathrm{~mL}\right), 1$ octadecene (18 mL), and $\mathrm{PbO}(4 \mathrm{mmol}, 0.8928)$ were added to a 3-neck round-bottom flask. In a separate flask 1-octadecene $(10 \mathrm{~mL})$ was added along with a stir bar. Both flasks were sealed with a stopcock, removed from the glovebox, and attached to a Schlenk line under nitrogen atmosphere. The flask containing $\mathrm{PbO}$ and oleic acid was heated to $120^{\circ} \mathrm{C}$ and left under stirring until the solution became clear. Separately, bis(trimethylsilyl)sulfide ( $2 \mathrm{mmol}, 0.421 \mathrm{~mL}$ ) was added to the second flask containing 1-octadecene $(10 \mathrm{~mL})$ using a syringe while maintaining an inert atmosphere. Next, the bis(trimethylsilyl)sulfide solution was injected into the lead oleate solution and left for 1 minute before heat was removed and the reaction was quenched with $10 \mathrm{~mL}$ of toluene. $\mathrm{PbS}$ QDs were transferred to $50 \mathrm{~mL}$ centrifuge tubes before they were precipitated from the reaction mixture with acetone (1:1 by volume) and subsequently centrifuged (5 minutes at 5000 RPM, Thermo Scientific Sorval ST16 Centrifuge with a Fiberlite F15 6x 100y rotor). The supernatant was decanted and the QDs were re-dispersed in toluene. A total of 4 precipitation/redispersion cycles were performed before the QDs were precipitated a final time, and the QD pellet was transferred into the glovebox. The QDs were ultimately re-dispersed in anhydrous hexane to a final concentration of $\sim 50 \mathrm{mg} / \mathrm{mL}$. 


\section{3) 3-Mercaptopropionic Acid Ligand Exchange}

$\mathrm{PbS}$ QDs capped with 3-MPA were prepared at a concentration of $2.5 \mathrm{mg} / \mathrm{mL}$ for further analysis. The QDs were prepared through a bi-phasic ligand exchange in a $15 \mathrm{~mL}$ centrifuge tube utilizing Oa-PbS QDs in hexane $(8 \mathrm{~mL}, 1.25 \mathrm{mg} / \mathrm{mL})$ layered above a lower polar phase $(4 \mathrm{~mL})$ of DMSO with $10 \% \mathrm{v} / \mathrm{v} 3-\mathrm{MPA}(1.2 \mathrm{M})$. After layering the two phases the tube was gently and repeatedly inverted until the QDs migrated completely to the bottom phase, typically after less than a minute. The bottom phase was transferred to a clean tube, centrifuged (12,000 RPM, $5 \mathrm{~min}$ ) as a precaution to remove any potential agglomerates, and the supernatant collected for further experiments; all aging experiments were done with solutions stored in glass vials.

\section{4) Nuclear Magnetic Resonance Spectroscopy}

Spectra were acquired on a $400 \mathrm{MHz}$ Bruker Avance 2+ NMR spectrometer with a $5 \mathrm{~mm}$ Broad Band Observe probe running TopSpin 2.1.6. Spectra were plotted separately using MestReNova NMR software. Quantification samples were performed in triplicate for error analysis. For aging of 3-MPA samples, concentrations were normalized to the original concentration of 3-MPA in solution via stoichiometric balance following our proposed reaction mechanism in Scheme 1. For Oa ${ }^{-}$quantification p-dichlorobenzene was used as an internal standard. In 3-MPA QD colloids the internal standard was added after exchange to prevent interference from any loss of the standard into the non-polar phase. 


\section{5) Optical Spectroscopy}

Absorption spectra were acquired in glass fluorimetry cells (Starna cells, 9F-Q-10-GL14-

C) using a $4 \mathrm{~mm}$ path length. For luminescence experiments, glass cells with reduced path lengths were constructed from microscope slides separated by glass-bead spacers $(<106 \mu \mathrm{m}$ or $<600 \mu \mathrm{m})$ in UV-cure epoxy (Loctite \#349). Absorption spectra were acquired utilizing a Shimadzu 3600 UV-Vis-NIR spectrophotometer in dual-beam mode with a solvent blank and the provided UVProbe software. Luminescence spectra were acquired with a StellarNet Dwarf-Star InGaAs NIR spectrometer and the provided Spectrawiz software. The InGaAs detector was calibrated using an incandescent bulb and the assumption that is was a perfect black-body emitter with a temperature of $2800 \mathrm{~K} .^{111}$ Spectra were collected with fiber-optic cable and excited using either a $<35 \mathrm{~mW} 780 \mathrm{~nm}$ NIR or $<40 \mathrm{~mW} 532 \mathrm{~nm}$ green laser for the $<600 \mu \mathrm{m}$ or $<106 \mu \mathrm{m}$ thick cells, respectively. Emission spectra were normalized by matching the absorbance of 3-MPA and $\mathrm{Oa}^{-}$capped QD sample at the excitation wavelength (780 or $\left.532 \mathrm{~nm}\right)$. The absorbance at the excitation wavelength was always lower than 0.1. Relative emission spectra were y-scaled by normalizing to the emission maximum of the $\mathrm{Oa}^{-}$capped QD sample.

\section{6) Solvent Absorption Correction of Emission Spectra}

Raw emission data taken in cuvettes for aging studies were corrected to account for the presence of strong DMSO absorption peaks in the emission window. The absorbance of the solvent was converted to a transmission value that was divided from the measured emission intensity to correct for lost signal. 


$$
\begin{aligned}
& T(\lambda)=10^{-A(\lambda)} \\
& I(\lambda)=\frac{I_{o b s}(\lambda)}{T(\lambda)}
\end{aligned}
$$

Where $\mathrm{T}, \mathrm{A}, \mathrm{I}$, and $\mathrm{I}_{\mathrm{obs}}$ are normalized transmission, absorbance, corrected emission intensity, and observed emission intensity, respectively. T, A, I, and $\mathrm{I}_{\mathrm{obs}}$ are all wavelength-dependent.

\section{D) Results}

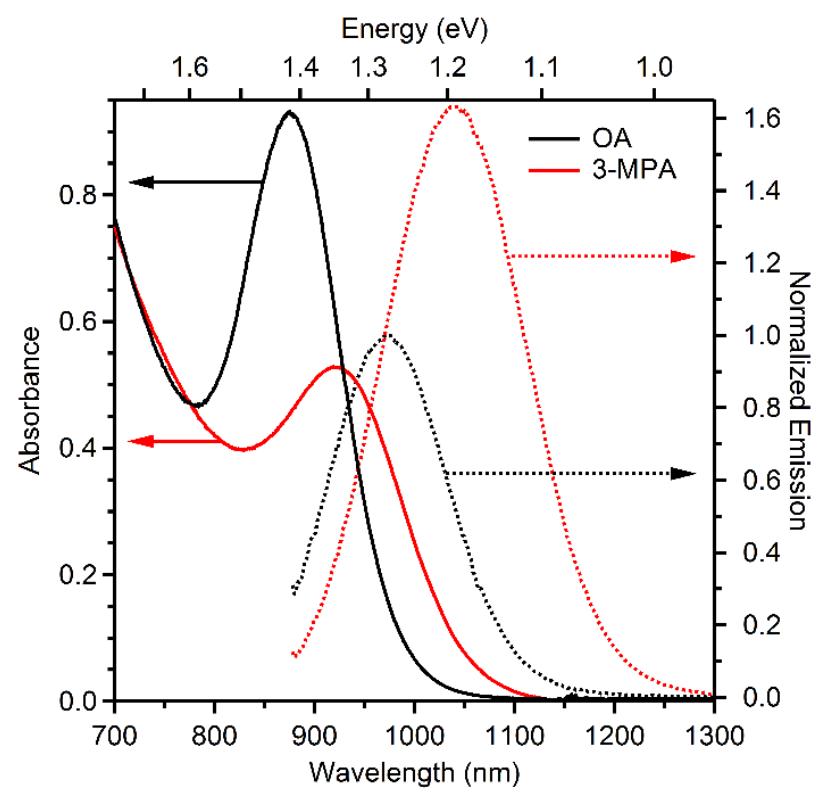

Figure III-1. Absorption (solid traces) and luminescence (dotted traces) spectra of colloidal 3-MPA exchanged PbS QDs in 10\% v/v 3-MPA in DMSO $(2.5 \mathrm{mg} / \mathrm{mL})$ and oleate capped PbS QDs in toluene. Samples were matched for absorption at the excitation wavelength $(\sim 2.5 \mathrm{mg} / \mathrm{mL})$. Absorption spectra were acquired in $4 \mathrm{~mm}$ cuvettes whereas emission spectra were acquired in glass cells with thicknesses $<106 \mu \mathrm{m}$ excited at $532 \mathrm{~nm}$.

Figure III-1 shows absorption and emission spectra for the original oleate capped QDs and freshly exchanged QDs. Oleate capped PbS QDs were suspended in toluene, 
exchanged QDs were suspended in 10\% v/v 3-MPA in DMSO. Both absorption and emission peaks redshifted, approximately $\sim 80 \mathrm{meV}$ for absorbance and $\sim 90 \mathrm{meV}$ for emission, upon ligand exchange. Phase transfer of the PbS QDs, and stability of the resulting solution, was dependent on the presence and amounts of all reagents and reactants. Oa-PbS QDs did not migrate to the DMSO phase in the absence of 3-MPA. Oa-PbS QDs did not migrate into neat 3-MPA. Upon dilution of the exchanged QD colloid both absorption and emission spectra underwent peak broadening and red-shifting that was not observed in Oa-PbS QD colloids. Oa-PbS QD samples displayed decreasing emission yield $(<10 \%$ emission intensity loss at the peak emission wavelength over 10 seconds during episodic data collection). This did not change significantly with reduced laser power and was not observed for exchanged QDs.

Table III-1. Peak fit data from emission experiments. Samples were taken in triplicate to obtain standard deviations on peak position, FWHM, and emission intensity.

\begin{tabular}{|c|c|c|c|c|c|c|}
\cline { 3 - 7 } \multicolumn{2}{c|}{} & \multicolumn{2}{c|}{$\mathrm{eV}$} & \multicolumn{2}{c|}{$\mathrm{nm}$} & \multicolumn{1}{c|}{} \\
\cline { 3 - 7 } \multicolumn{2}{c|}{} & Peak Position & FWHM & Peak Position & FWHM & Amplitude (Counts) \\
\hline $532 \mathrm{~nm}$ & $3-\mathrm{MPA}$ & $1.19 \pm 0.00$ & $0.19 \pm 0.00$ & $1045 \pm 4$ & $168 \pm 3$ & $29379 \pm 4364$ \\
\cline { 2 - 7 } & Oa $^{-}$ & $1.28 \pm 0.00$ & $0.18 \pm 0.00$ & $975 \pm 0$ & $139 \pm 0$ & $21853 \pm 655$ \\
\hline $780 \mathrm{~nm}$ & $3-\mathrm{MPA}$ & $1.19 \pm 0.00$ & $0.18 \pm 0.01$ & $1049 \pm 1$ & $159 \pm 5$ & $7470 \pm 531$ \\
\cline { 2 - 7 } & Oa $^{-}$ & $1.28 \pm 0.00$ & $0.19 \pm 0.00$ & $976 \pm 2$ & $145 \pm 2$ & $10426 \pm 1843$ \\
\hline
\end{tabular}

Table III-1 shows peak fitting data from emission experiments using the two excitation wavelengths. For each set an $\mathrm{Oa}-\mathrm{PbS}$ QD sample was matched to absorbance values within 10 mO.D. at the excitation wavelength before samples were loaded into the emission cells. All spectra were acquired in triplicate with independent emission cells for each replicate. All peak fitting was performed on spectra acquired as quickly as possible upon exposure of the cells to the excitation light. 


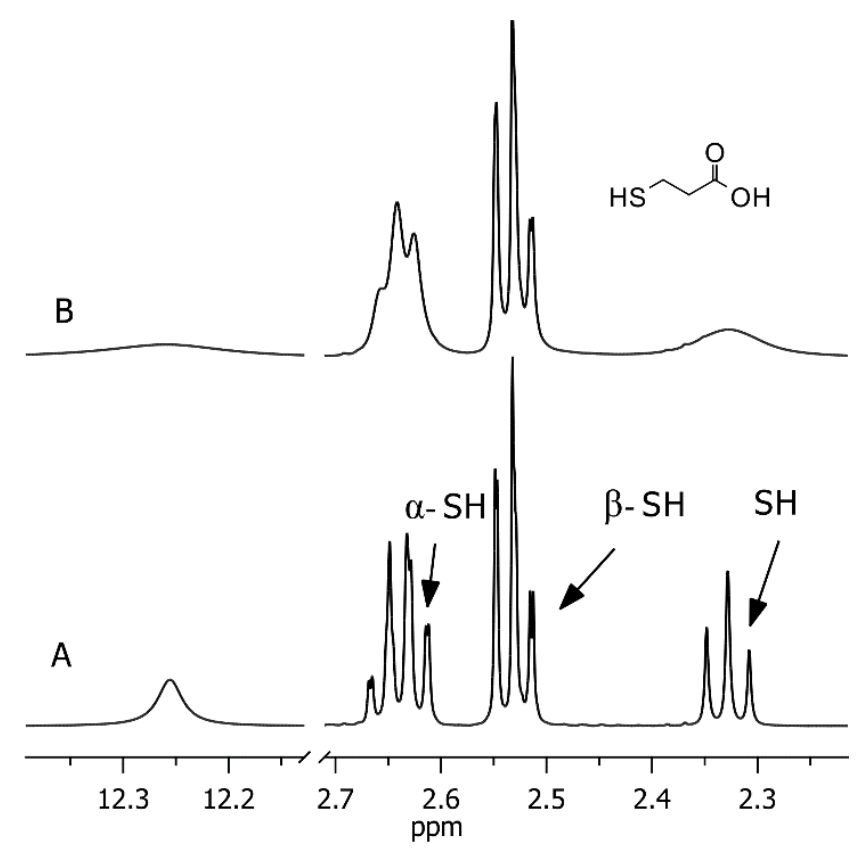

Figure III-2. ${ }^{1} \mathrm{H}-\mathrm{NMR}$ spectra of 3-MPA with and without the presence of PbS QDs. (A) 10\% v/v 3-MPA in DMSO-D 6 and (B) 10\% 3-MPA exchanged PbS QDs in 10\% v/v 3-MPA in DMSO-D 6 .

Figure III-2 (A) shows the solution NMR spectrum of 3-MPA in DMSO; the assignments were verified by 2-dimensional correlation spectroscopy (Figure S III-1). Figure III-2(B) shows the spectrum of exchanged QDs in 3-MPA/DMSO. The thiol peak, typically presumed to be the binding functionality to $\mathrm{Cd}$ and $\mathrm{Pb}$ chalcogenide $\mathrm{QDs},{ }^{112,113}$ was significantly broadened along with mildly reduced signal. The protons on the methylene $\alpha$ to the thiol moiety changed from a well-defined quartet to a broad triplet. There was no significant signal reduction from the $\mathrm{COOH}$ functionality ( 12.3 ppm) upon coordination of 3-MPA to the surface of the QDs despite the singlet $\mathrm{COOH}$ peak broadening significantly. 


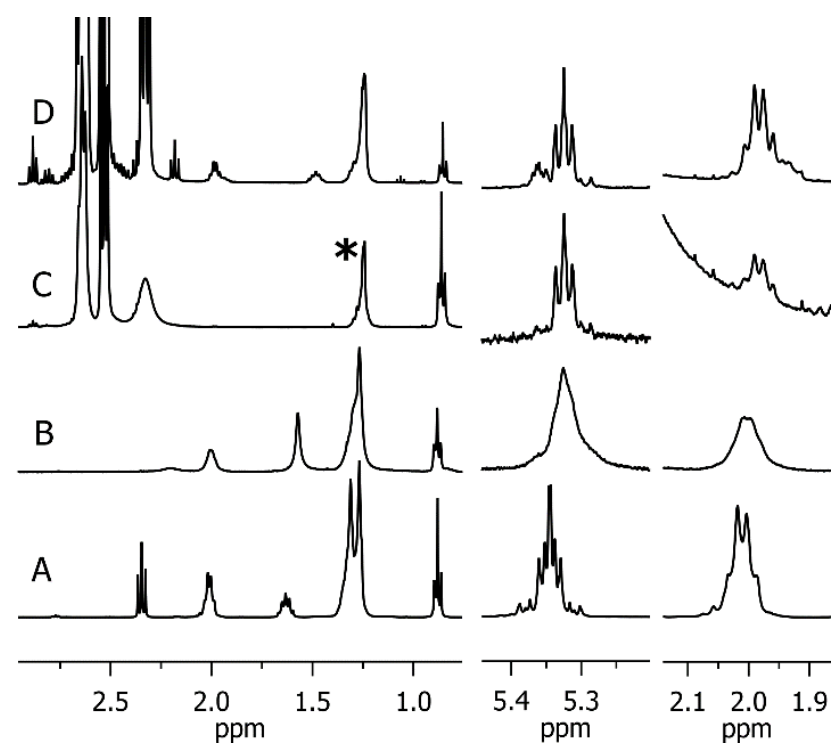

Figure III-3. ${ }^{1} \mathrm{H}-\mathrm{NMR}$ spectra of oleyl species under varying experimental conditions indicating interaction, or lack thereof, with PbS QDs. (A) $\mathrm{OaH}$ in $\mathrm{CDCl}_{3}$, (B) Oa-PbS QDs in $\mathrm{CDCl}_{3}$, (C) 3-MPA capped $\mathrm{PbS}$ QDs in 10\% v/v 3-MPA in DMSO-D 6 , and (D) OaH in 10\% v/v 3-MPA in DMSO-D 6 . Peaks marked “*” derive significant intensity from hexane transferred into the DMSO phase in (C). Resolvable peaks from oleyl species include the alkene ( $\sim 5.33 \mathrm{ppm})$ and $\alpha$-alkene $(\sim 1.98 \mathrm{ppm})$ hydrogens observable in the DMSO phase, other peaks from oleyl species are masked by n-hexane or hexane isomers.

Figure III-3 shows 1H-NMR spectra of $\mathrm{OaH}$ in $\mathrm{CDCl} 3, \mathrm{Oa}^{-}$capped $\mathrm{PbS}$ QDs in $\mathrm{CDCl} 3$, 3-MPA capped PbS QDs in 10\% v/v 3-MPA/DMSO-D 6 , and $\mathrm{OaH}$ in 10\% v/v 3-MPA in DMSO-D6. Residual OaH can be observed in the 3-MPA/DMSO phase after exchange (C). The total amount of remaining $\mathrm{OaH}$ in the 3-MPA/DMSO phase was approximately $28 \%$ based on the integrated intensity of the alkene peaks ( $~ 5.33 \mathrm{ppm})$ and $29 \%$ when compared to the $\alpha$-alkene peaks $(\sim 1.98 \mathrm{ppm})$. Full assignment of $\mathrm{OaH}$ is provided in Figure S III-2. Concentrations were quantitatively determined relative to an internal standard of 1,4-dichlorobenzene. Large peaks, marked “*” at 1.24 and $0.86 \mathrm{ppm}$ in the 3- 
MPA capped PbS QD sample belong to hexane solubilized in the MPA/DMSO phase during bi-phasic transfer.

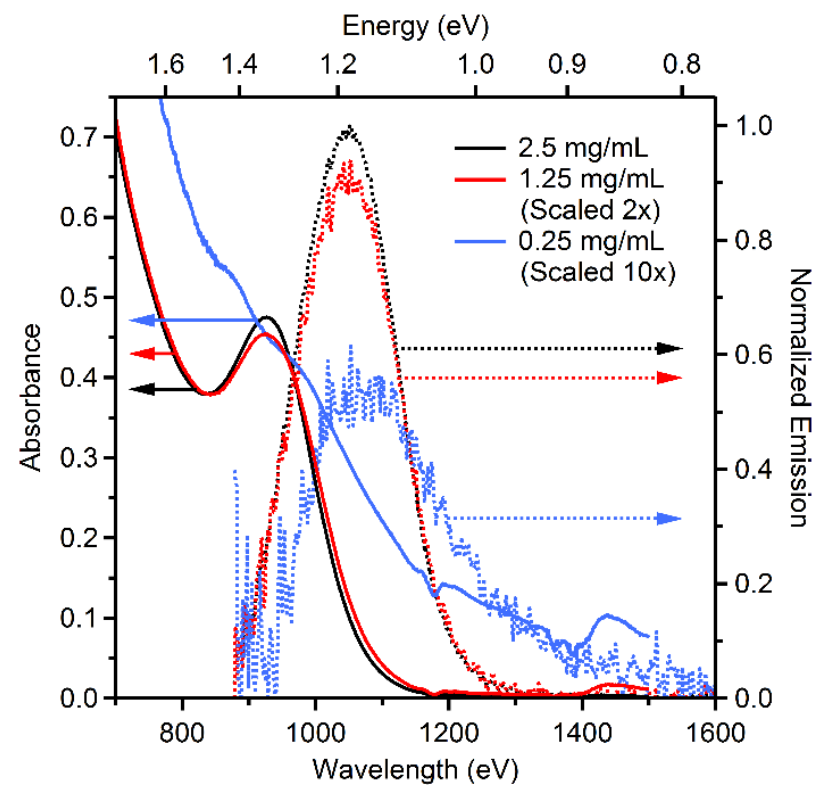

Figure III-4. Absorption (solid trace) and emission (dotted trace) spectra of 3-MPA capped PbS QDs at different $\mathrm{PbS}$ QD concentrations. Spectra of diluted samples have been scaled by an amount equivalent to their respective dilution factor to demonstrate evolution of peak shape and red-shifting. 3-MPA QDs were suspended in 10\% v/v 3-MPA in DMSO. Absorption spectra were acquired in $4 \mathrm{~mm}$ cuvettes whereas emission spectra were acquired in $<106 \mu \mathrm{m}$ glass emission cells.

Figure III-4 shows the effect of dilution with 10\% v/v 3-MPA in DMSO on optical spectra of 3-MPA capped PbS QDs originally prepared at $2.5 \mathrm{mg} / \mathrm{mL}$. Dilution to 1.25 $\mathrm{mg} / \mathrm{mL}$ resulted in slight broadening of both absorption and emission profiles that was also observed while preparing 3-MPA capped PbS QDs at lower concentrations directly. Further dilution $(0.025 \mathrm{mg} / \mathrm{mL})$ resulted in the loss of a distinct observable excitonic transition and significantly broadened emission. 
Optical spectra of exchanged PbS QD colloids evolved over time (Figure III-5). The absorption spectra baseline increased over time consistent with an increasingly scattering sample. The peak centered around $\sim 955 \mathrm{~nm}$ appeared to evolve into distinct peaks, at the original position and at a lower wavelength. The emission spectra underwent both broadening and red-shifting over the same time period.

The emission spectra for Figure III-5 only were collected using $4 \mathrm{~mm}$ path length cuvettes despite the increased self-absorption of emitted light. This was done because the epoxy of the thin cells degraded over $24-48$ hours when in contact with DMSO. Aging performed in the glovebox gave qualitatively similar results except that luminescence intensities were lower by approximately a factor of 2 .
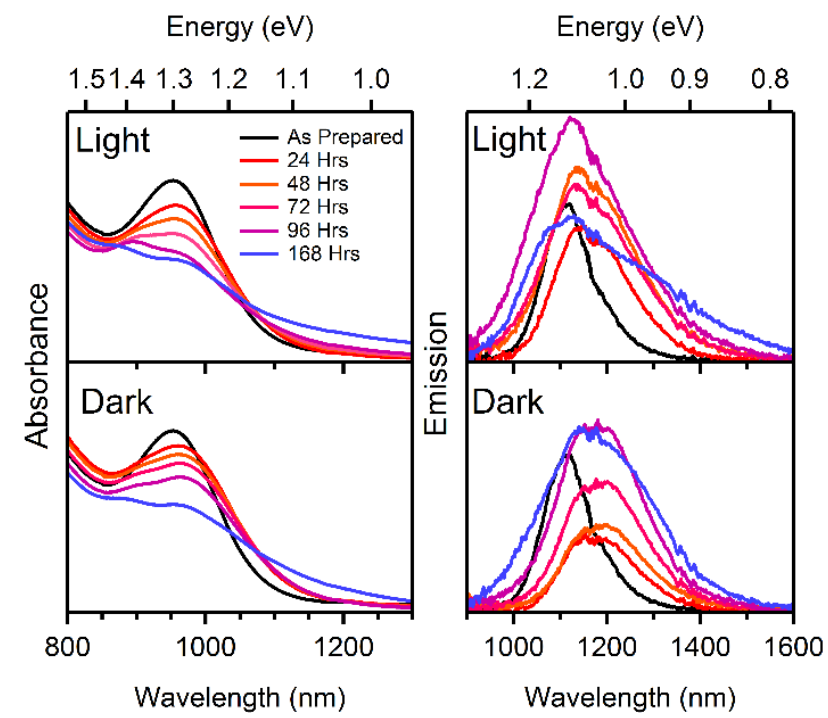

Figure III-5. Absorption (A) and luminescence (B) spectra of 3-MPA capped PbS QDs at $2.5 \mathrm{mg} / \mathrm{mL}$ across a seven day period of aging under both dark and light conditions stored in an ambient environment.

DMSO has been shown to participate in oxidative reactions with thiols; a previously proposed reaction scheme is shown below (Scheme 1). ${ }^{101,102}$ In DMSO-D 6 the reaction would generate a dimer of 3-MPA, dithiodipropionic acid (dTdPA, shown in Figure S 
III-3), deuterated dimethylsulfide, and protonated water. Solutions of $10 \% \mathrm{v} / \mathrm{v} 3-\mathrm{MPA}$ in DMSO-D 6 were aged. A water peak (not displayed) was observed to grow along with the dTdPA peak at a quantitative ratio consistent with the proposed reaction scheme stoichiometry. Conversion rate was not affected by illumination. Both the thiol and the peak $\beta$ to the thiol ( 2.33 and $2.53 \mathrm{ppm}$, respectively) shifted as the solution aged but did not evidence any difference in quantification of dimer formation (Figure S III-4).

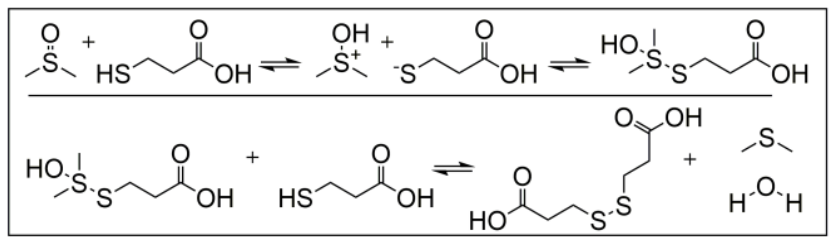

Scheme III-1. Oxidative dimerization of 3-MPA to dTdPA.

DMS formed in DMSO- $\mathrm{D}_{6}$ would, according to the proposed scheme, be deuterated and not appear in the $1 \mathrm{H}$ spectral region. To test the proposed formation of DMS, 3-MPA was also aged in protonated DMSO to allow evolution of a signal from DMS (Figure S III-5). DMS formation was verified. 


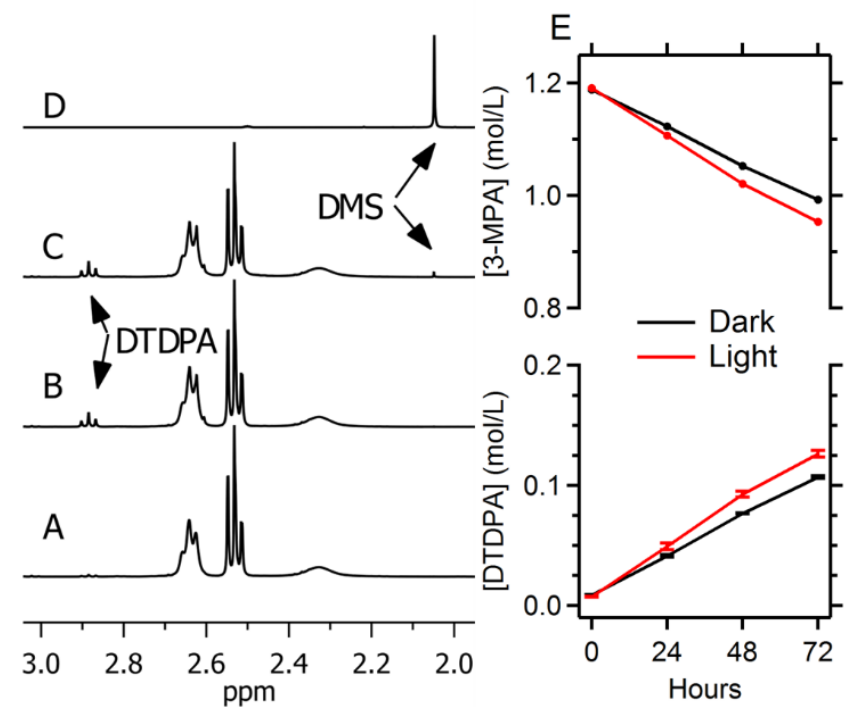

Figure III-6. 1H-NMR spectra of 3-MPA exchanged QDs in DMSO-D 6 under different aging conditions. (A) Freshly prepared, (B) aged 24 hours in the dark, and (C) aged 24 hours exposed to light. (D) NMR spectrum of DMS in DMSO-D . $_{\text {. }}$ (E) shows the difference in molecular conversion between light and dark environments when PbS QDs are present.

Figure III-6 shows the evolution of 1H-NMR spectra from QDs stored in the dark and under illumination. Here, illumination affected the reaction; the 3-MPA thiol peak decreased more rapidly and the dTdPA increased more rapidly under illumination. Furthermore, DMS- $\mathrm{H}_{6}$ formation was observed, as opposed to DMS- $\mathrm{D}_{6}$ formation as proposed by Scheme 1 (Figure S III-6).

\section{E) Discussion}

3-MPA capped PbS QD colloids were prepared by biphasic ligand exchange that replaced the original oleate ligand. 3-MPA is presumed to be an X-type ligand that undergoes proton transfer to bound oleate, releasing free oleic acid. ${ }^{114}$ 3-MPA was present in large excess ( 210 fold excess concentration) compared to surface bound oleate in the original 
QD samples, and PbS QDs interacted with a majority of 3-MPA on the time scale of NMR experiments. This was evidenced by the observed broadening of the peak assigned to $\mathrm{S}-\mathrm{H}$ and the change in splitting from a quartet to a triplet of the methylene $\alpha$ to the thiol at $2.64 \mathrm{ppm}$ in Figure III-2. ${ }^{104,115}$ Broadened resonances in 1H-NMR of bound species have been attributed to an inhomogeneous magnetic environment on the QD surface, ${ }^{116}$ and peak broadening is less pronounced for species farther from the QD surface. ${ }^{108,117}$ The change in splitting from a quartet to a triplet of the methylene $\alpha$ to the thiol suggests dynamically exchanging thiol hydrogen. The observed broadening of the peak assigned to $\mathrm{S}-\mathrm{H}$ was dependent on the presence of QDs and thus not a result of the formation of reaction intermediates proposed in Scheme 1. Thus, 3-MPA interacted with the QDs by the thiol group. This observation is in line with previous studies of binding of short carboxy-terminated thiols, including 3-MPA, to CdS that have been shown through IR studies to bind through the thiol moiety. ${ }^{112,113}$

The presence of QDs also affected the signal attributed to $\mathrm{COOH}$. However, there is no unambiguous evidence of binding by the $\mathrm{COOH}$ moiety. The peak assigned to $\mathrm{COOH}$ broadened in the presence of QDs but did not decrease in intensity. There was no direct coupling between the carboxylic acid proton on 3-MPA and the protons $\beta$ to the thiol. As such, these protons would not see any change in split pattern with the protonation state of the carboxylate group. However, Oa has been shown to bind to CdSe by the deprotonated carboxylate. ${ }^{107}{ }^{1} \mathrm{H}-\mathrm{NMR}$ spectra of Oa capped QDs reconfirmed this to be the case with $\mathrm{PbS}$ as well (Figure $\mathrm{S} \mathrm{III-2).} \mathrm{From} \mathrm{this,} \mathrm{we} \mathrm{can} \mathrm{expect} \mathrm{the} \mathrm{COOH}$ moiety on 3-MPA to bind in the deprotonated carboxylate form. The spectral features here assigned to S-H 
and $\mathrm{COOH}$ have previously been linked to dynamically stabilized CdTe QDs with fast exchanging alkylamine ligands. ${ }^{118}$ Similar to our observations, those colloids became unstable at lowered ligand concentrations.

Residual oleic acid (Figure III-3) was detected by 1H-NMR at reduced levels ( 28$29 \%$ of original concentration). A closer examination of the 3-MPA capped PbS QDs in DMSO- $\mathrm{D}_{6}$ revealed that the peaks assigned to the oleic acid alkene hydrogens and the hydrogens $\alpha$ to the alkene hydrogens showed resolvable peak splitting. The positions and splitting of the peaks were consistent with free $\mathrm{OaH}$ in 10\% v/v 3-MPA in DMSO-D 6 , but in contrast to $\mathrm{Oa}^{-}$bound PbS QDs (Figure S III-2) and PbSe QDs. ${ }^{104}$ Thus, the signals that were observed were consistent with the presence of free oleic acid. Taken together, the data is consistent with colloidally stable PbS QDs with a dynamic 3-MPA ligand shell with free oleic acid present in the solution.

As-prepared 3-MPA-capped PbS QDs were luminescent. The luminescence of other metal chalcogenide QDs capped with 3-MPA has been reported previously. 3-MPA capped CdSe QDs have been studied in detail. ${ }^{119-121}$ 3-MPA to CdSe association was found to lead to emissive deep trap formation on the surface of the QDs. While various ligands have been shown to modulate PL both negatively and positively after replacing native ligands on CdSe QDs, thiols were consistently seen to decrease photoluminescence. ${ }^{122,123}$ In a related study with alkane thiols of varying length capping the surface of PbS QDs, surface mobility of capping molecules was found to correlate to trap-state emission. ${ }^{124}$ PL from solids prepared by centrifugally casting 3-MPA QDs has been studied, but no comparison was made to emission intensity from similar films of 
oleate capped PbS QDs. ${ }^{53}$ It is interesting to note that the 3-MPA-capped PbS QDs studied herein luminesced with intensities comparable to oleate capped PbS QDs and showed relatively narrow emission when freshly prepared (Figure III-1). A relative luminescence quantum yield for 3-MPA capped vs. oleate capped PbS QDs was calculated using equation $1 .^{125}$

Equation III-1.

$$
\Phi_{s}=\Phi_{r} \frac{F_{s}\left(1-10^{-A_{r}}\right) n_{s}^{2}}{F_{r}\left(1-10^{-A_{s}}\right) n_{r}^{2}}
$$

Where $\Phi_{\mathrm{s}}$ is the luminescence quantum yield of the sample; $\Phi_{\mathrm{r}}$ is the luminescence quantum yield of the reference; $F_{s}$ and $F_{r}$ are the integrated intensities of the sample and reference spectra, respectively; $\mathrm{A}_{\mathrm{s}}$ and $\mathrm{A}_{\mathrm{r}}$ are the absorbances at the excitation wavelength for the sample and reference, respectively; $n_{s}$ and $n_{r}$ are the refractive indices of the sample and reference solutions, respectively. To understand the effect of ligand exchange on luminescence intensity $\Phi_{\mathrm{r}}$ is taken to equal 1 . The will give $\Phi_{\mathrm{s}}$ as luminescence quantum yield relative to $\mathrm{Oa}-\mathrm{PbS}$ QDs. For freshly prepared samples the shape of luminescence spectra from 3-MPA and Oa-PbS QDs are similar, and peak intensities are substituted in place of peak areas. Typical absorbances of the two samples at the excitation wavelength were $<0.1$. Finally, the refractive index of toluene (used here due to lower volatility in thin cell emission experiments) was taken as 1.4969; the refractive index of $10 \% \mathrm{v} / \mathrm{v} 3-\mathrm{MPA} / \mathrm{DMSO}$ was measured as 1.4783 using a refractometer. This gives $\Phi_{\mathrm{s}}$ as 1.31 or 0.69 using the $106 \mu \mathrm{m}\left(\lambda_{\mathrm{ex}}=532 \mathrm{~nm}\right)$ and $600 \mu \mathrm{m}$ $\left(\lambda_{\mathrm{ex}}=780 \mathrm{~nm}\right)$ luminescence cells, respectively. While the numbers are not in absolute agreement, they both suggest that the 3-MPA capped QDs luminesced with intensities 
comparable to oleate capped PbS QDs. An additional factor, not accounted for here, that will have affected the measurement is the effect of refractive index on luminescence lifetime. ${ }^{29}$ Luminescence lifetime decreases as refractive index increases. In the present study, the reference solvent for Oa-PbS QDs had a higher refractive index, compared to sample solvent for 3-MPA capped QDs. A decreased luminescence lifetime would increase luminescence yield by more effectively competing with non-radiative relaxation mechanisms. The result in this case would be an underestimation of the relative luminescence quantum yield of the sample compared to the reference since the $10 \% \mathrm{v} / \mathrm{v}$ 3-MPA/DMSO had a lower measured refractive index than toluene.

Attempts at diluting or cleaning the colloid modified the optical properties of the as prepared QDs. 3-MPA QDs could not be prepared by bi-phasic exchange with 1\% v:v 3MPA in DMSO; the QDs precipitated when lower concentrations of 3-MPA were used. Furthermore, although diluting the QDs while maintaining a constant concentration of 3MPA in DMSO resulted in changes to the optical properties consistent with agglomeration, no aggregates were observed by dynamic light scattering studies (Figure S III-7). Concentration dependent aggregation has been observed previously for other QD-ligand systems. ${ }^{126}$ It was shown that ligand-QD affinity, solvent dielectric constant, solvent Lewis acidity and basicity, and ligand solvation propensity all determined whether colloidal stability could be achieved. It is assumed that some solvent system exists in which 3-MPA capped PbS QDs can be prepared without a large excess of free 3MPA. 
The optical spectra of 3-MPA capped PbS QD colloids changed over time and was correlated to the formation of dimerized 3-MPA, dTdPA. The rate of dTdPA formation was modulated by the presence of QDs and in that case also illumination; illumination did not matter in the absence of quantum dots. The change in concentration of 3-MPA over 72 hours in the absence of QDs was approximately $0.2 \mathrm{M}$. The change in concentration of 3-MPA over 72 hours that can be attributed to the presence of QDs under illumination was approximately $0.038 \mathrm{M}$. Using quantitative NMR data the concentration of $\mathrm{Oa}^{-}$was approximately $0.005 \mathrm{M}$. Assuming that $1 \mathrm{Oa}^{-}$corresponds to 1 surface site the change in concentration of 3-MPA was greater than the concentration of QD surface sites. It follows that QD surface sites were not passivated when 3-MPA was converted to dTdPA; each surface site was able to promote several dimerization events. The formation of non-deuterated DMS in DMSO-D 6 suggests that the reaction mechanism involving QDs was significantly different than proposed in Scheme 1 for oxidation of 3-MPA in DMSO. The observed influence of light on dithiol formation is consistent both with studies of PbS QDs stabilized by short ionic ligands in water, ${ }^{127}$ and studies of CdSe nanocrystals photo-oxidizing thiols. Disulfides have decreased affinity for CdSe nanoparticles compared to thiol groups. ${ }^{128}$ Photocatalytically formed dithiols have been observed previously with CdSe QDs, leading to QD precipitation. ${ }^{129} \mathrm{dTdPA}$ also has the possibility of binding to QD surfaces via the carboxylate group and may additionally be capable of cross-linking QDs. To examine dTdPA as a ligand, PbS QDs were directly exposed to dTdPA under identical conditions used for biphasic ligand exchange with 3-MPA. The QDs immediately aggregated at the liquid/liquid interface 
without successful suspension in either phase. Centrifugation of aged samples of 3-MPA capped QDs recovered absorption spectra more closely resembling freshly prepared solutions but still consistent with a significant fraction of agglomerated quantum dot species present. Taken together, one possibility these observations suggest is that slow formation of dTdPA in solution or on QD surfaces results in eventual precipitation from suspension due to cross linking of QDs; Aggregates did form when the colloids were aged, consistent with observed formation of dTdPA in solution. This is consistent with the observed degradation of colloidal stability that was correlated to dithiol formation as well as particle aggregation, seen in Figure S III-7. Consistent with this, centrifugation of aged samples recovered absorption spectra more closely resembling freshly prepared solutions but still consistent with a significant fraction of agglomerated quantum dot species present.

\section{F) Conclusions}

Oa-capped PbS QDs were converted to colloidal PbS QDs stabilized by a dynamic 3MPA ligand shell. 3-MPA was shown to interact with PbS QDs by the thiol group. Binding of 3-MPA to the surface of the QDs via the carboxylate moiety was likely but could not be demonstrated definitively. 3-MPA capped QDs were colloidally stable only with a substantial excess of 3-MPA; this, taken with the population averaged changes to the 1H-NMR spectra of 3-MPA exchanged QDs, was consistent with fast exchange where 3-MPA readily detaches and reattaches to the QD surface as a weakly bound ligand. As prepared 3-MPA capped QDs luminesced with intensities comparable to oleate capped PbS QDs. Optical spectra of 3-MPA exchanged QDs evolved over the course of 
days with both absorption and emission spectra maintaining resolvable signal. This aging was correlated to oxidation of 3-MPA to dTdPA. Conversion of 3-MPA to dTdPA was observed to be independent of illumination in the absence of QDs but modulated by illumination when QDs were present. The ability to perform ligand exchange and QD colloidal stability were found to be highly dependent on solution identity and component (QD/ligand) ratios. 


\section{G) Supplementary Material}

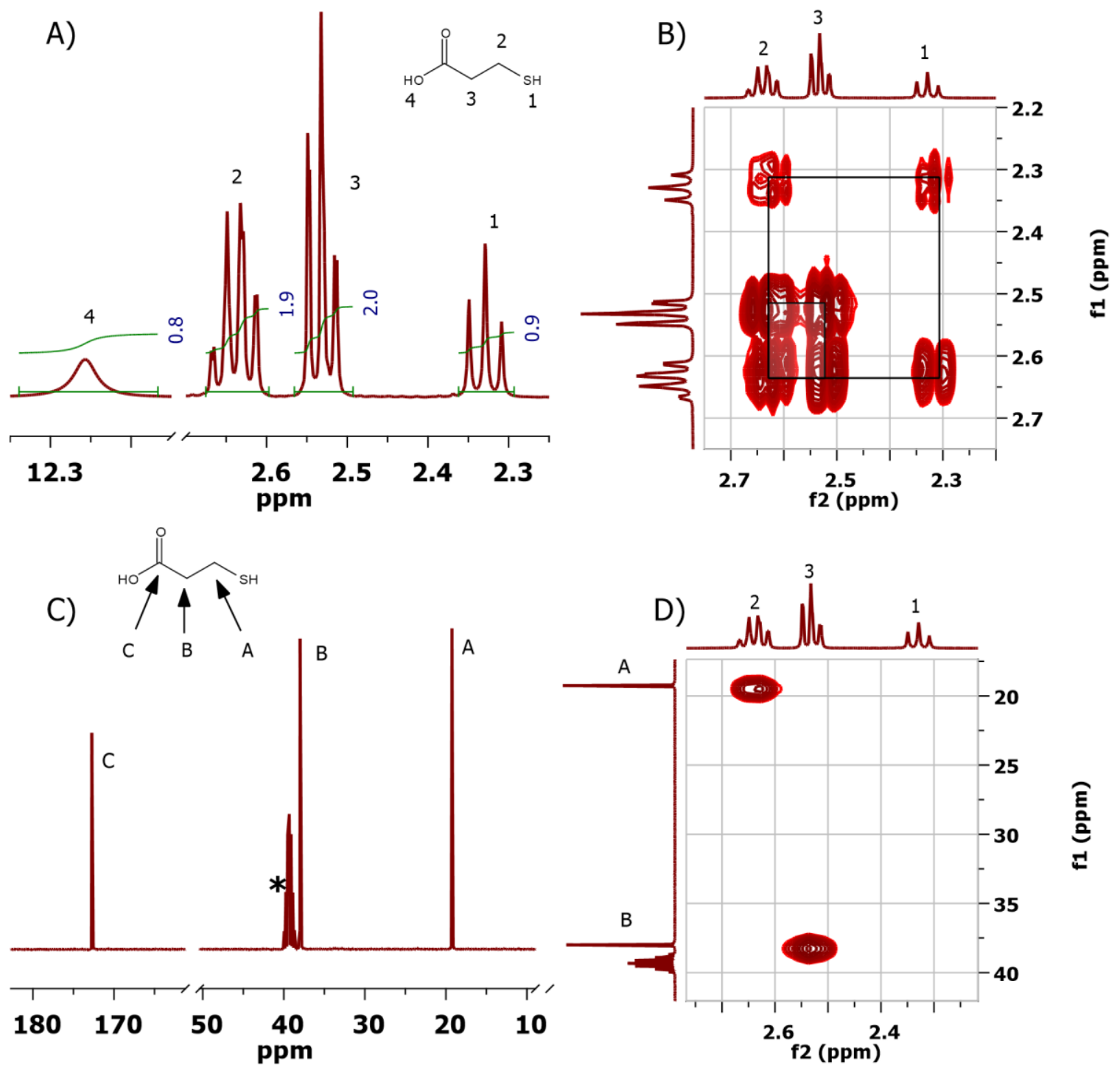

Figure S III-1. NMR spectra for full assignment of 3-MPA in DMSO-D 6 . (A) One dimensional ${ }^{1} \mathrm{H}-\mathrm{NMR}$,

(B) ${ }^{1} \mathrm{H}-{ }^{1} \mathrm{H}$ Correlation spectrum (COSY) showing cross peaks between coupled proton environments on 3-

MPA, (C) ${ }^{13} \mathrm{C}-\mathrm{NMR}$ spectrum, and (D) ${ }^{1} \mathrm{H}^{-13} \mathrm{C}$ heteronuclear single-quantum correlation spectrum (HSQC).

* in (C) shows signal from the solvent, DMSO. There is no correlation to the proton environment at $\sim 2.3$ in

(D) since the proton at that position is attached to sulfur instead of carbon. 


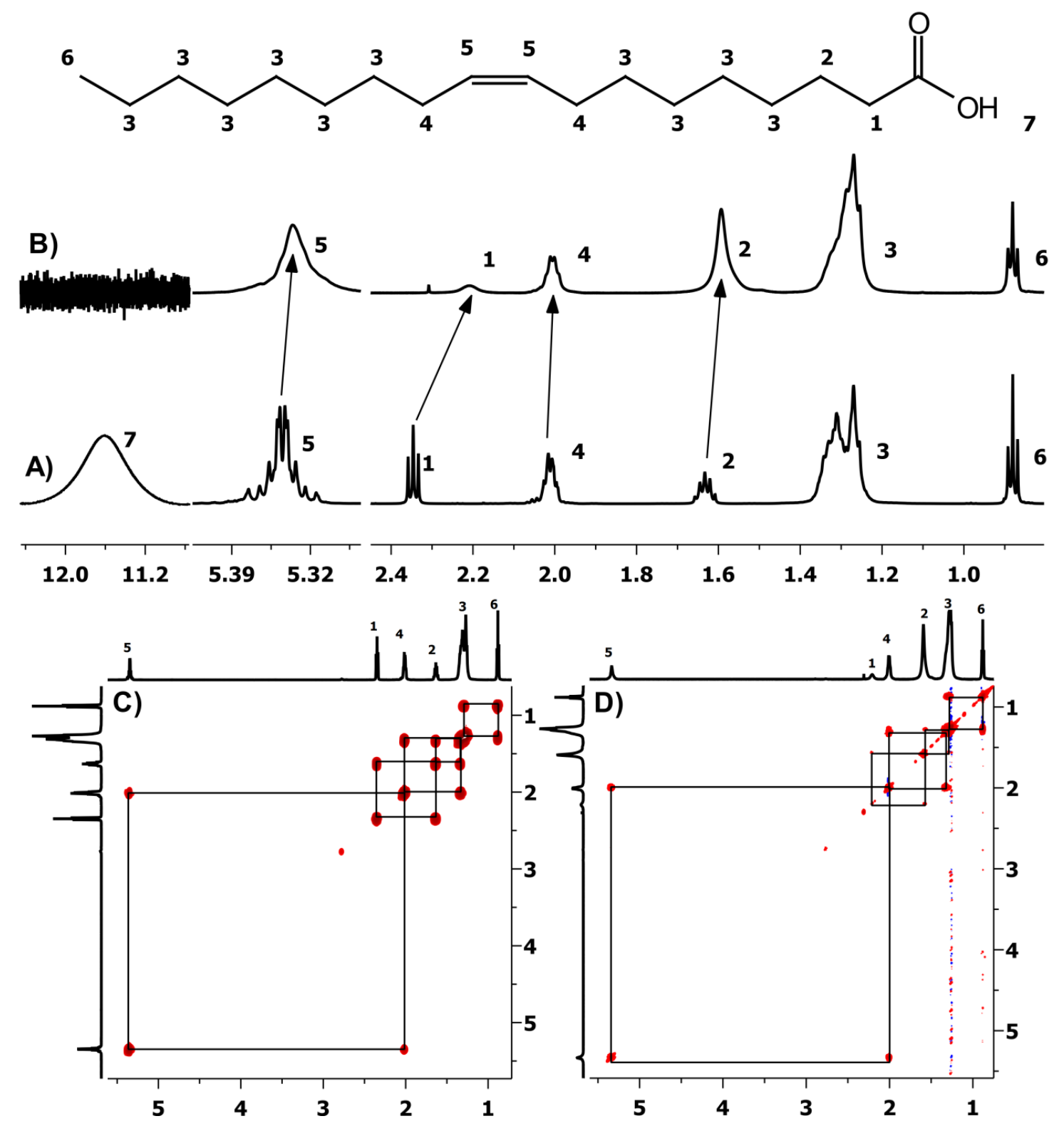

Figure S III-2. ${ }^{1} \mathrm{H}-\mathrm{NMR}$ of $\mathrm{OaH}(\mathrm{A})$ and $\mathrm{Oa}-\mathrm{PbS}$ QDs (B) in $\mathrm{CDCl} 3$ with assignments labeled relative to the displayed structure of oleic acid. Of particular note is the absence of any $\mathrm{COOH}$ signal in the $\mathrm{Oa}-\mathrm{PbS}$ QD spectrum, indicating binding through a carboxylate functionality which has been demonstrated elsewhere for oleic acid on CdSe QDs. ${ }^{107}$ Spectra were acquired in $\mathrm{CDCl}_{3 .}{ }^{1} \mathrm{H}-{ }^{1} \mathrm{H}$ COSY spectrum of $(\mathrm{C})$ oleic acid and (D) Oa-PbS QDs in $\mathrm{CDCl}$. Each different ppm region of the 1-dimensional 1H spectra has been y-scaled independently to focus on the peak of interest in that region. 


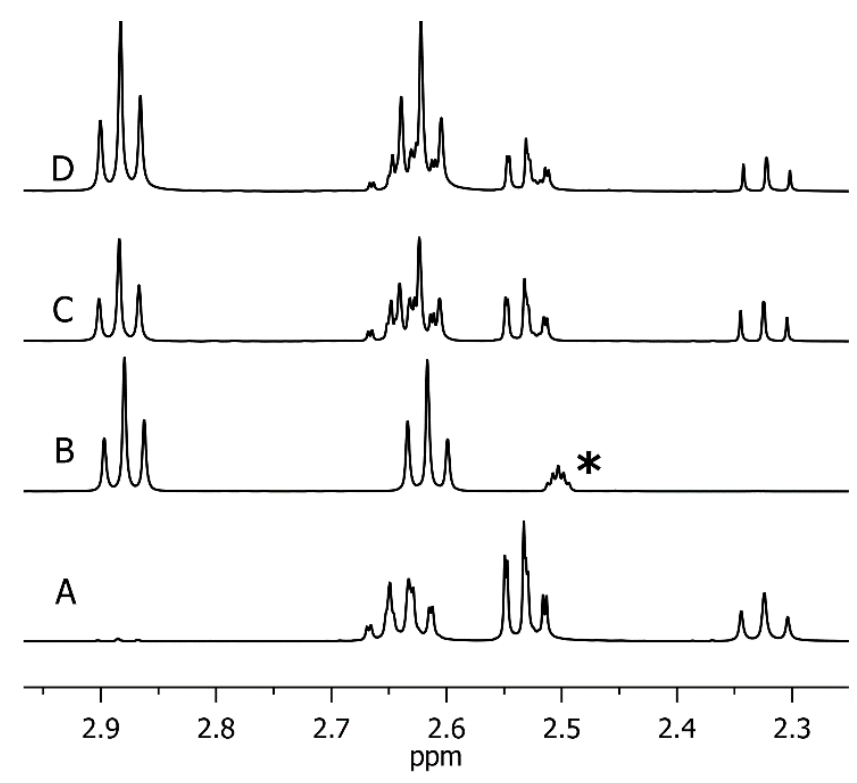

Figure S III-3. ${ }^{1} \mathrm{H}-\mathrm{NMR}$ Spectra in DMSO-D 6 showing identification of dTdPA growth during aging. (A) 3-MPA, (B) dTdPA, (C) Aged 3-MPA, (D) Aged 3-MPA spiked with dTdPA. DMSO can be seen in (B) at $2.5 \mathrm{ppm}$ marked and has been labeled (*).

Figure S III-3 shows ${ }^{1} \mathrm{H}-\mathrm{NMR}$ of 3-MPA (A) and the dimer, 3,3'-dithiodipropionic acid (dTdPA) (B). A solution of 3-MPA in DMSO-D 6 that had been aged for 336 hours (2 weeks) showed peaks corresponding to the dimer (C). The origin of the additional peaks in the aged sample was verified by spiking said sample with dTdPA. This increased the relative intensity of the new peaks $v s$ those belonging to 3-MPA without introducing additional peaks (D). 

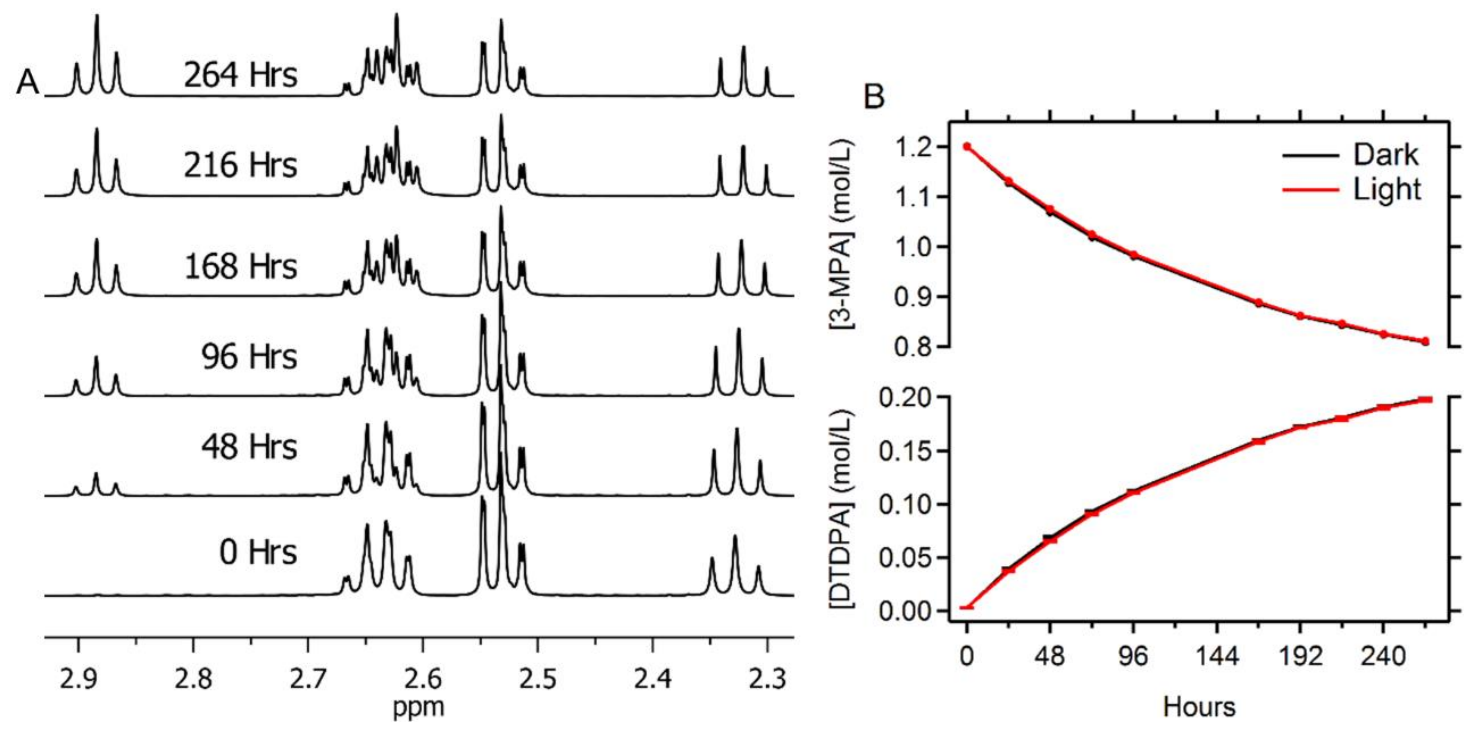

Figure S III-4. (A) ${ }^{1} \mathrm{H}-\mathrm{NMR}$ spectra of 3-MPA in DMSO-D 6 during aging and (B) calculated concentrations of 3-MPA and dTdPA.

The composition of solutions of 10\% 3-MPA in DMSO was further investigated as a function of time. Figure S III-4 shows that two triplets emerge, at $\sim 2.88 \mathrm{ppm}$ and $\sim 2.62$ ppm. The second peak overlaps with the signal from the methylene $\alpha$ to the thiol moiety of 3-MPA. The peak at $\sim 2.88$ ppm evolves at a $\sim 1: 2$ quantified ratio of growth to signal loss compared to the peak at $\sim 2.53 \mathrm{ppm}$. 


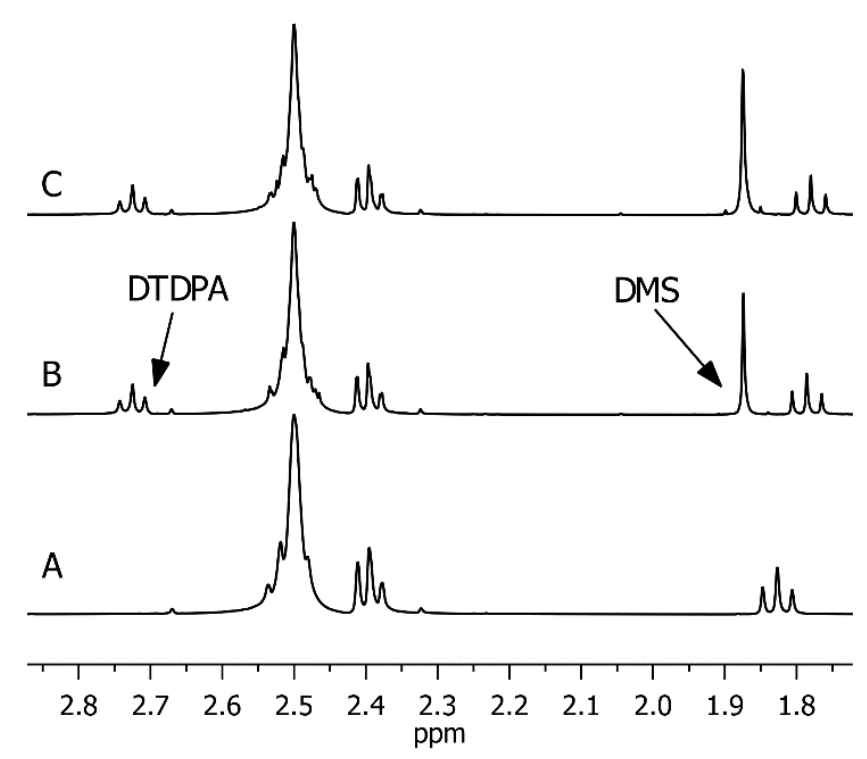

Figure S III-5. ${ }^{1} \mathrm{H}-\mathrm{NMR}$ spectra of 3-MPA aging in DMSO. A) Freshly prepared 50\% v/v of 3-MPA and protonated DMSO and the same sample B) after 24 hours. C) shows the same sample as in B) with an addition of DMS that would increase [DMS] by $1 \mathrm{M}$. DMSO- $\mathrm{H}_{6}$ is visible as a large peak at $2.5 \mathrm{ppm}$.

Figure S III-5 shows an emergent signal consistent with DMS formation (singlet, 1.88 ppm) and spiking the sample with neat DMS showed increased signal without introducing additional peaks. 


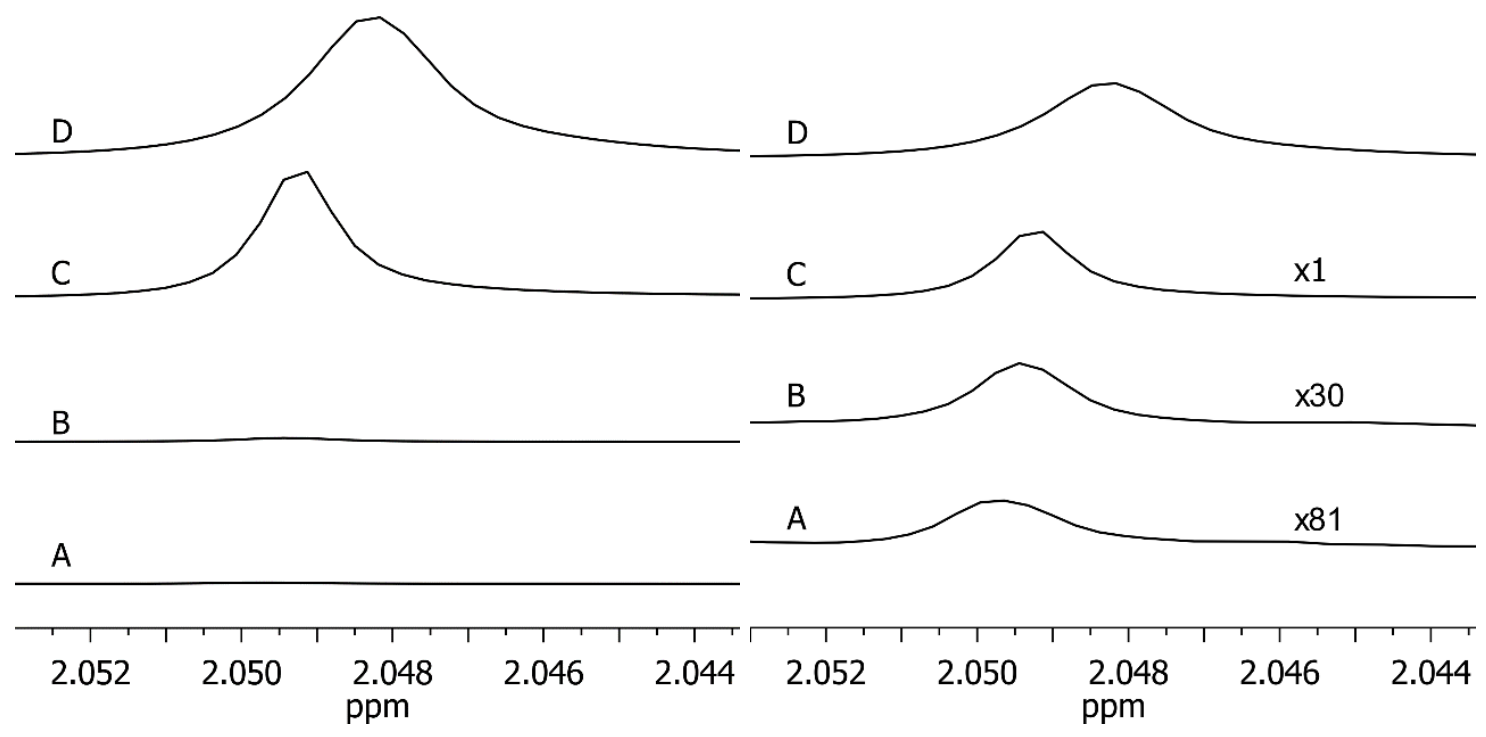

Figure S III-6. ${ }^{1}$ H-NMR spectra of 3-MPA exchanged QDs in DMSO-D 6 . (A) Freshly prepared, (B) aged 24 hours in the dark, and (C) aged 24 hours exposed to light. (D) NMR spectrum of DMS in DMSO-D 6. Samples with formed DMS (A-C) are at similar concentrations of 3-MPA and QDs, the signal intensity reflects different amounts of formed DMS while the DMS in DMSO-D 6 sample (D) was prepared independently. The figure on the right shows scaled spectra (A-C) to demonstrate small changes in chemical shift with changing concentration. This effect is also observed for the SH peak in Figure S III-4 (2.325 ppm). 


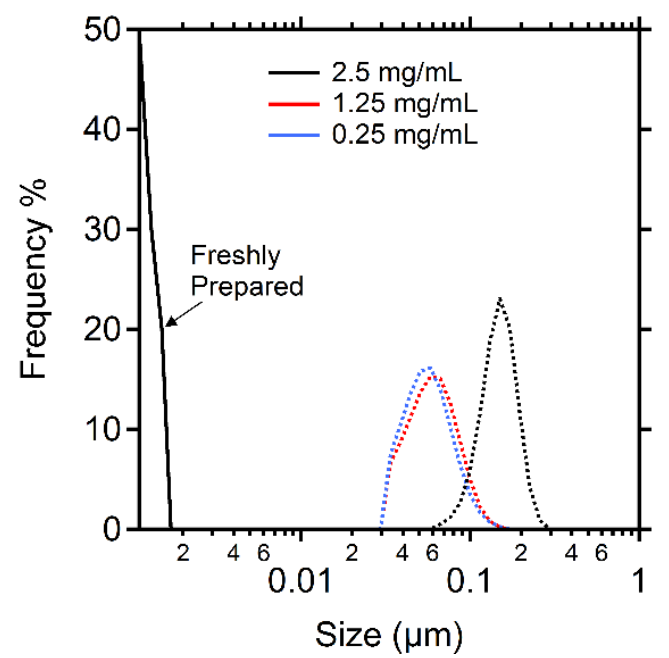

Figure S III-7. Dynamic light scattering of freshly prepared (three overlapping solid lines) and $24 \mathrm{hr}$ aged (dotted lines) samples of 3-MPA QDs in 10\% v/v 3-MPA in DMSO-D 6 at different QD concentrations.

Figure S III-7 shows particle size analysis of freshly prepared samples. The samples at $2.5,1.25$, and $0.25 \mathrm{mg} / \mathrm{mL}$ were free from aggregates as resolved by DLS. After 24 hours formed aggregates were observed by DLS. 


\title{
IV) COLLOIDAL 3-MERCAPTOPROPIONIC ACID CAPPED LEAD SULFIDE QUANTUM DOTS IN A LOW BOILING POINT SOLVENT
}

\begin{abstract}
A) Background
1) Photovoltaic device preparation using Colloidally Suspended 3-MPA capped PbS QDs in DMSO
\end{abstract}

After successfully preparing a colloidal "ink" of 3-MPA capped PbS QDs in DMSO, ${ }^{100}$ attempts at direct deposition and fabrication of photoactive devices $\left(\mathrm{TiO}_{2} / \mathrm{QD}\right.$ heterojunction solar cells) were made. A typical device, following the architecture described in Chapter II-B-IV was the model for these attempts. Direct deposition proved difficult for a number of reasons: DMSO is a non-volatile solvent that is not amenable to evaporative spin-coating, the solvent was too viscous and did not wet the surface of $\mathrm{TiO}_{2}$ substrates used as a wide bandgap acceptor material, the solvent proved difficult to remove under vacuum and did not provide conformal films. 


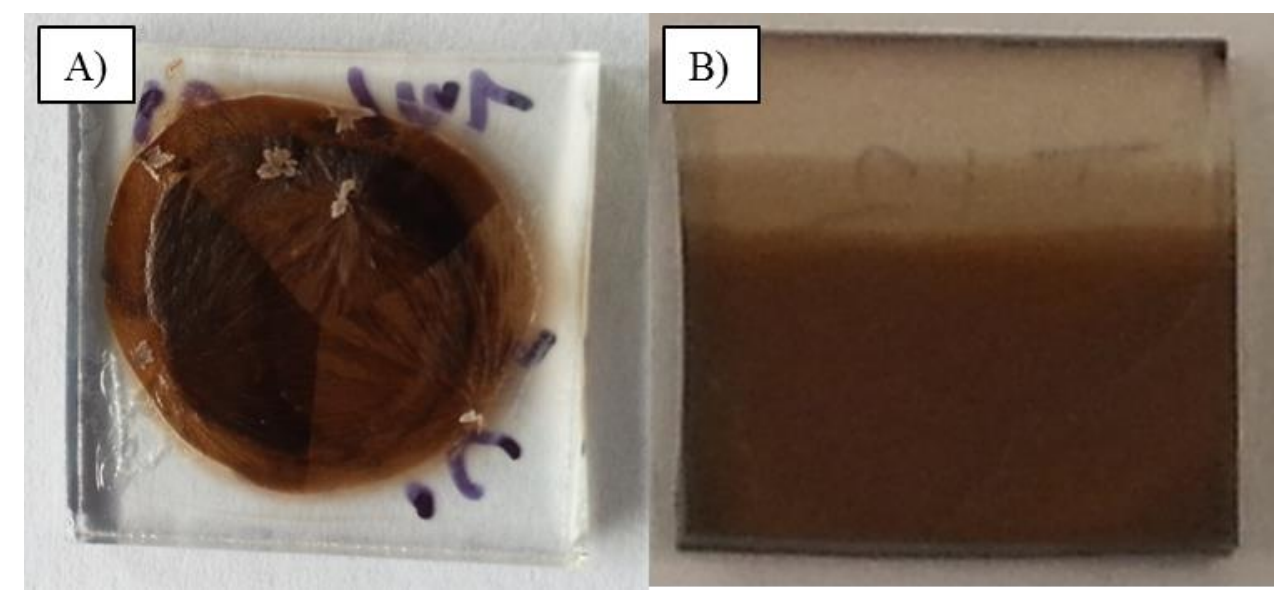

Figure IV-1. Comparison of 3-MPA capped PbS QD films on a transparent $\mathrm{TiO}_{2} /$ Fluorine doped $\mathrm{SnO} / \mathrm{SiO}_{2}$ substrate. Similar total amounts of 3-MPA capped QDs were deposited on each substrate. A) shows a vacuum deposited film of pre-exchanged QDs while B) shows a standard in situ exchanged film deposited through spin coating.

Figure IV-1 shows a vacuum deposited film of 3-MPA capped PbS QDs with visible cracking from the deposition process. This is compared to a conformal film deposited via standard in situ spin coating deposition that shows homogenous coating of the substrate. 


\section{Energy $(\mathrm{eV})$}

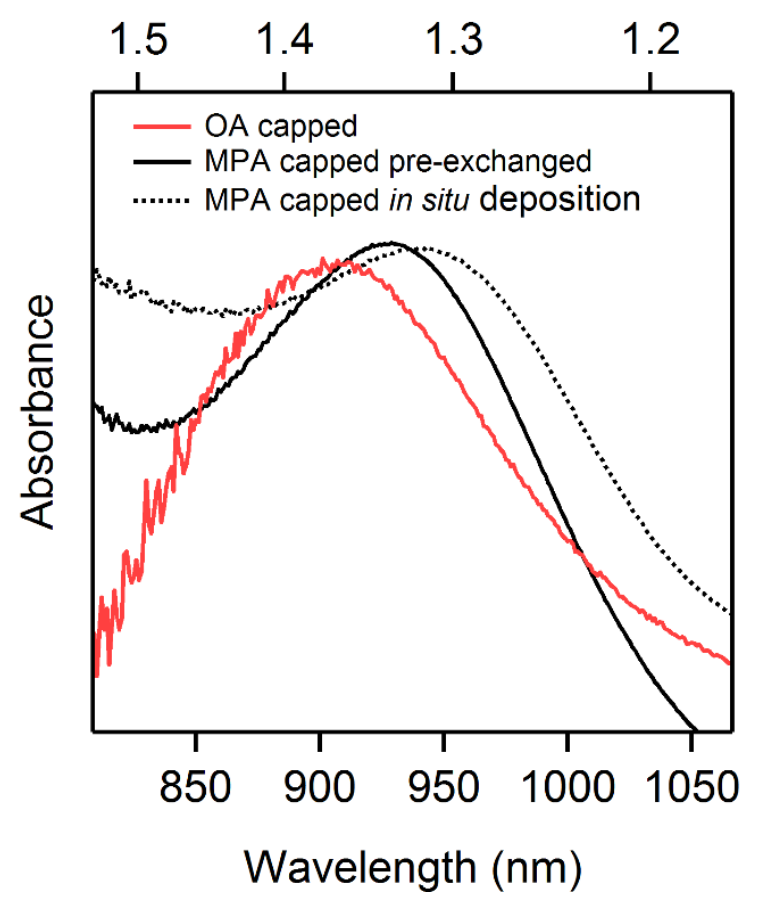

Figure IV-2. Absorption spectra of deposited films. OA capped PbS QD films were deposited directly by spin coating. Pre-exchanged 3-MPA capped PbS QD films were deposited via vacuum deposition. In situ exchanged 3-MPA capped PbS QD films were deposited via spin coating as described previously. Baseline differences are attributed to inhomogeneous substrate induced scattering.

Figure IV-2 shows that both types of films maintained a well resolved excitonic peak indicating that QDs survive the vacuum deposition process without destruction of their tuned properties. Red-shifting of excitonic absorption peaks upon cross-linking of QDs has been observed previously and is consistent with the difference between the two 3MPA capped PbS QD samples; ${ }^{64,130-132}$ that is, pre-encapsulation with 3-MPA may preclude the ability to cross-link extensively and thus may affect energetic coupling that leads to red-shifting in films. 


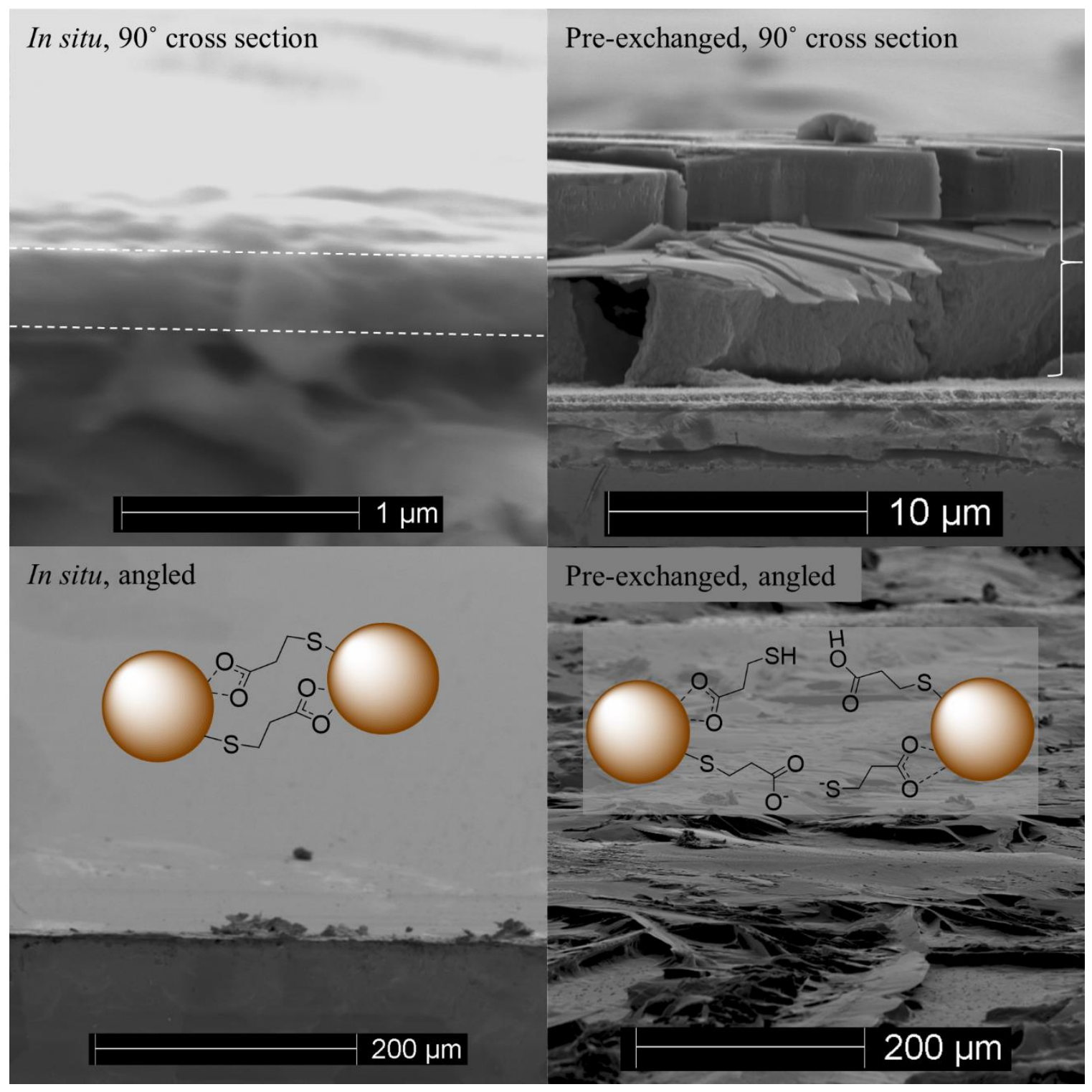

Figure IV-3. SEM micrographs of 3-MPA capped PbS QD films on $\mathrm{TiO}_{2}$ substrates. Cross sectional SEM (top) and angled (bottom) micrographs of an in situ deposited film and a vacuum deposited film using preexchanged 3-MPA capped PbS QDs suspended in DMSO. White lines in the top images highlight the PbS QD film atop the substrate.

Figure IV-3 shows scanning electron micrographs of pre-exchanged 3-MPA capped PbS QD films vacuum deposited from $\mathrm{DMSO}$ onto $\mathrm{TiO}_{2} /$ Fluorine doped $\mathrm{SnO} / \mathrm{SiO}_{2}$ substrates that revealed extensive cracking and disruption of the QD film. Films were dramatically 
thicker than expected given the mass of PbS QDs deposited over a measured area; contrasting this is the thickness of an in situ film deposited using a similar amount of QDs that are expected to be extensively crosslinked due to the bifunctional nature of 3MPA. Inter-QD distance is a large determinant for energy transfer between QDs with greater distances resulting in poorer electronic coupling. ${ }^{81,133,134}$ Additionally, the use of ligands with terminal moieties that contain dissociable protons leads to the possibility of electrostatic separation increasing inter-QD distances in films; while speculative as to its role in the large volume of deposited QD films, it was noted that colloids of 3-MPA capped PbS QDs in DMSO were subject to electrostatic separation while suspended. E.g., small droplets of DMSO containing 3-MPA capped PbS QDs could be drawn from solution using a charged surface such as a rubber balloon that had been vigorously rubbed with felt.

Despite these problems, photoactive devices were successfully fabricated. Figure IV-4 shows summary optoelectronic performance of the best performing prototype from a directly deposited 3-MPA capped PbS QD photoactive device. 

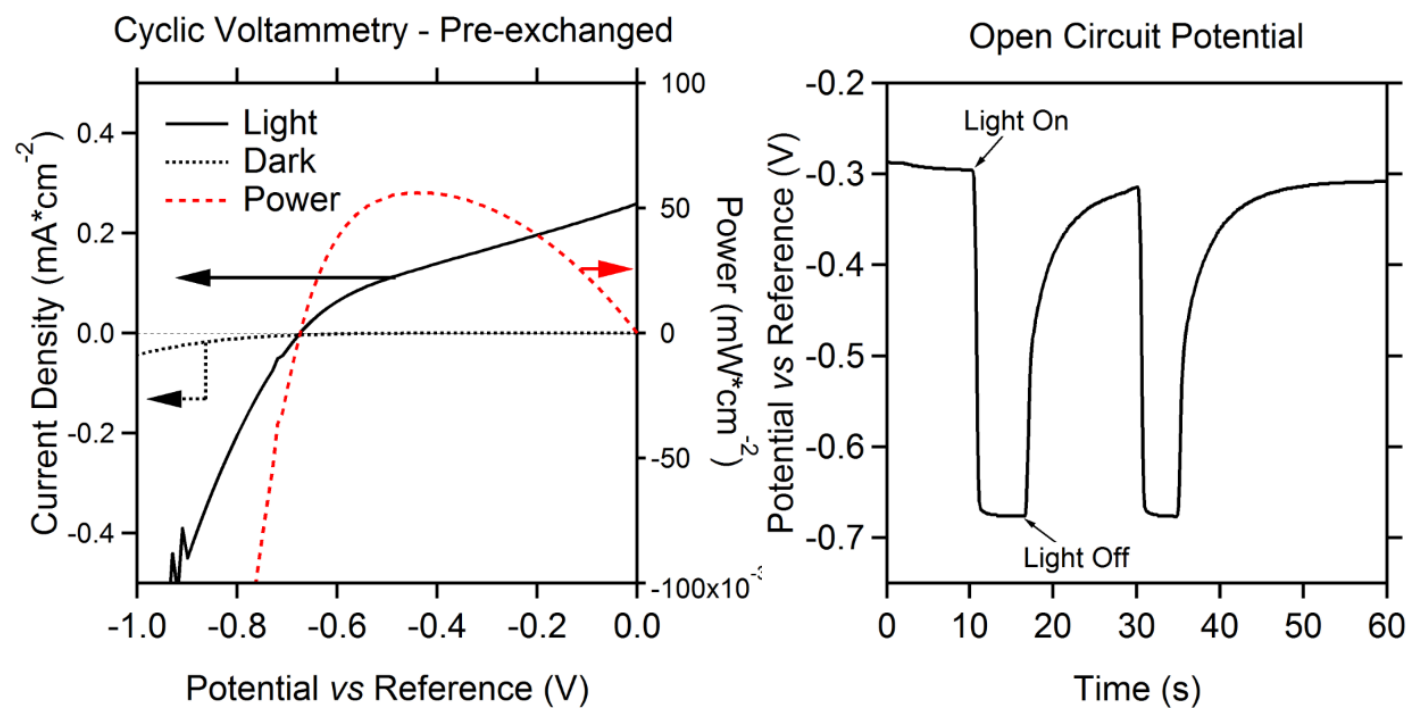

Figure IV-4. Cyclic voltammetry and open circuit potential of a directly deposited 3-MPA capped PbS QD photovoltaic device.

Prototype devices had a notably large apparent open circuit potential from cyclic voltammetry experiments, typical "benchmark" devices do not exceed $\sim 450-500 \mathrm{mV}$ under illumination. It was observed that a significant portion $(\sim 300 \mathrm{mV})$ of the total potential $(\sim 680 \mathrm{mV})$ was present in the dark and thus not from a photo-origin.

Nevertheless, the device was photo-switchable despite the aforementioned film formation problems.

In contrast to this is the performance of a standard in situ exchanged PbS QD photovoltaic device as seen in Figure IV-5. 

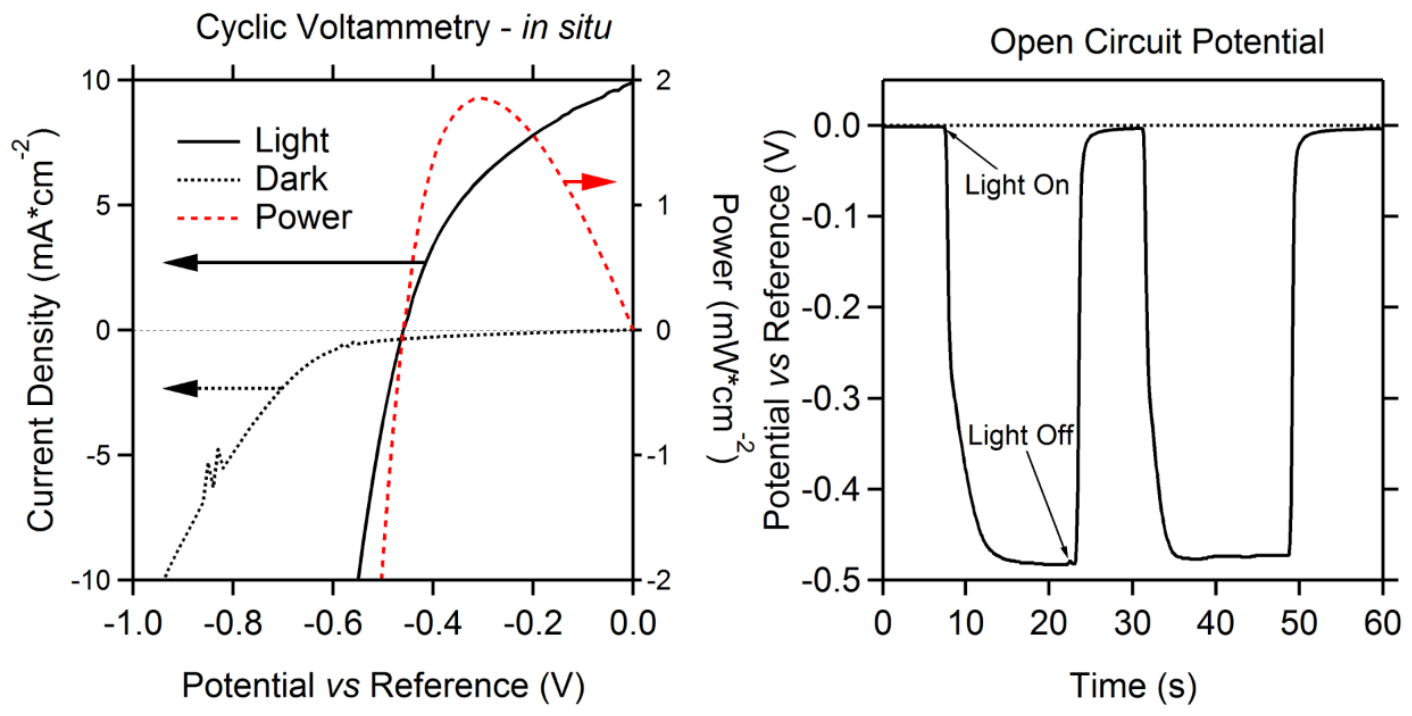

Figure IV-5. Cyclic voltammetry and open circuit potential of an in situ deposited 3-MPA capped PbS QD photovoltaic device.

This is normal behavior from a standard "benchmarking" device. The true discrepancy between the two is best observed when the performance from both devices is plotted on same scale axes as seen in Figure IV-6. 

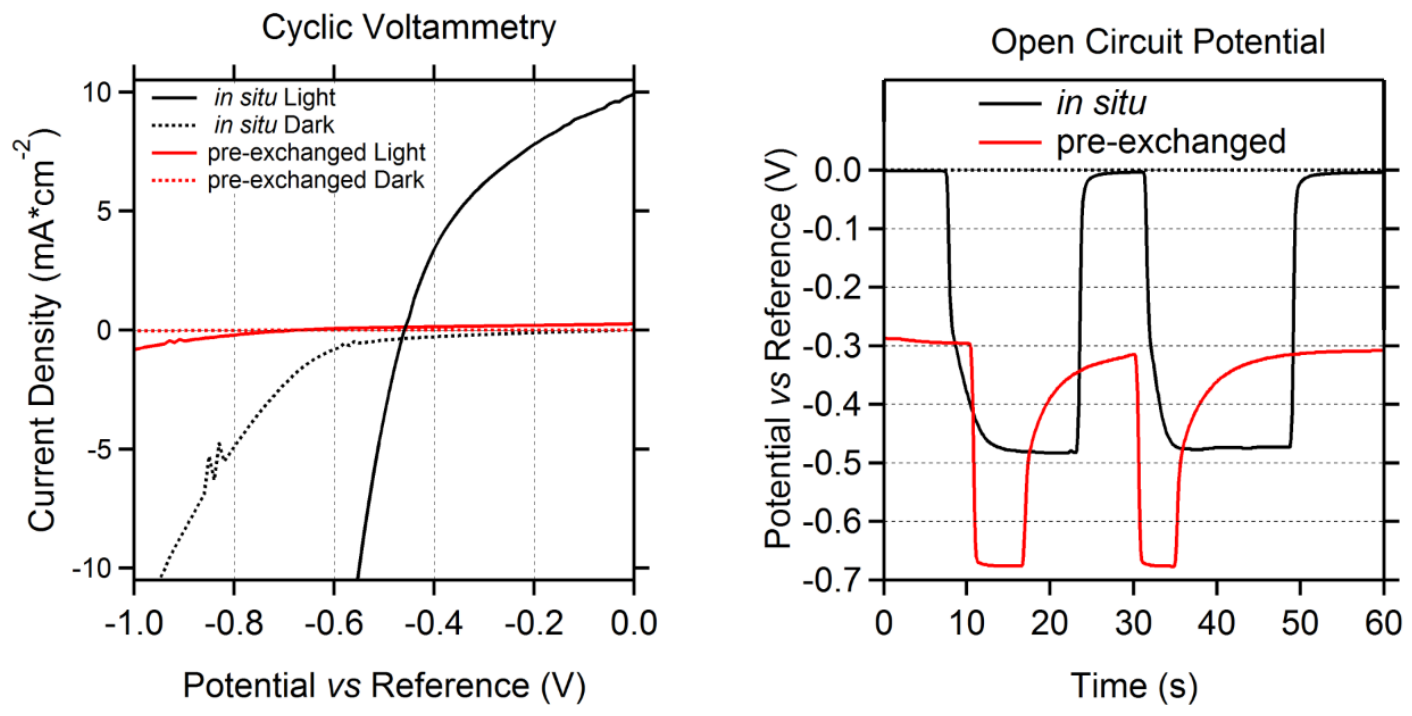

Figure IV-6. Simultaneously plotted cyclic voltammetry and open-circuit potential from prototype and in situ films.

While photoswitchability was achieved, prototype devices using the as formulated 3MPA PbS QD suspension in DMSO were exceedingly substandard. The inability to provide densely packed QD films using a vacuum assisted deposition process prompted the decision to attempt to formulate QD suspensions in solvents that were more amenable to direct ambient deposition using evaporative solvent loss, i.e. volatile organic solvents.

\section{2) Colloidal Stabilizers}

All previous attempts at solvent switching with 3-MPA capped PbS QDs to alternate solvents with comparable solvent parameters resulted in immediate aggregation of the QDs and subsequent settling of the suspension. Mixing solvent with DMSO, even at volume ratios of $1 \%$ had the same effect. Literature reports at this time suggested that increased acidity of labile protons on ligands intended to replace natively bound oleate on PbS QDs led to more successful exchange. ${ }^{87}$ Separately, it has been suggested in 
literature that ligand exchange in polar solvents causes ready desorption of bound species ultimately leading to agglomeration. ${ }^{135}$ In these reports, the addition of an organic base (triethylamine and oleylamine, respectively) reportedly increased the binding strength of the ligands by perturbing the acid base equilibria of the ligand to favor conjugate base formation of the incoming ligand.

Initial experiments with sec-butylamine, shown in Chart IV-1, as a stabilizing additive revealed that OA-QDs exposed to 3-MPA that had agglomerated could be resuspended upon addition of the amine with excellent recovery of resolved excitonic absorption and emission spectra. Figure IV-7 shows that secBA itself induced a mild blue-shift in absorbance spectra and significantly decreased emission yield of $\mathrm{OA}-\mathrm{PbS}$ QDs.

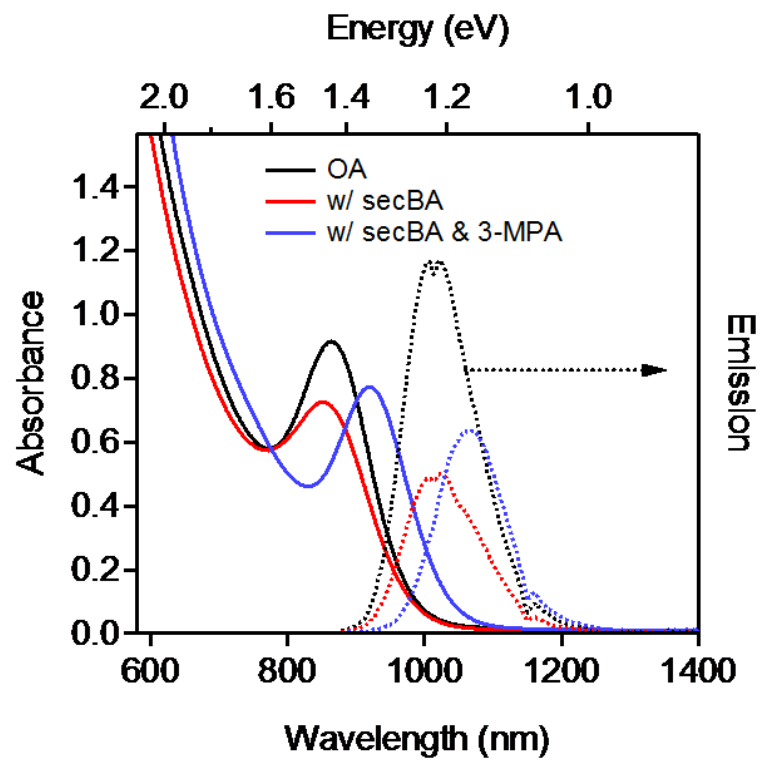

Figure IV-7. Absorption and emission spectra of OA-PbS QDs after exposure to solutions of secBA and 3MPA in $\mathrm{CHCl}_{3}$. 
Amines are normally observed to effect blue-shifts in optical spectra of QD suspensions, even when they do not induce ligand exchange. ${ }^{121,123}$ Additionally, while amines are possible QD ligands they are typically thought to be less preferential for binding metal chalcogenide QD surfaces relative to thiols. ${ }^{136}$

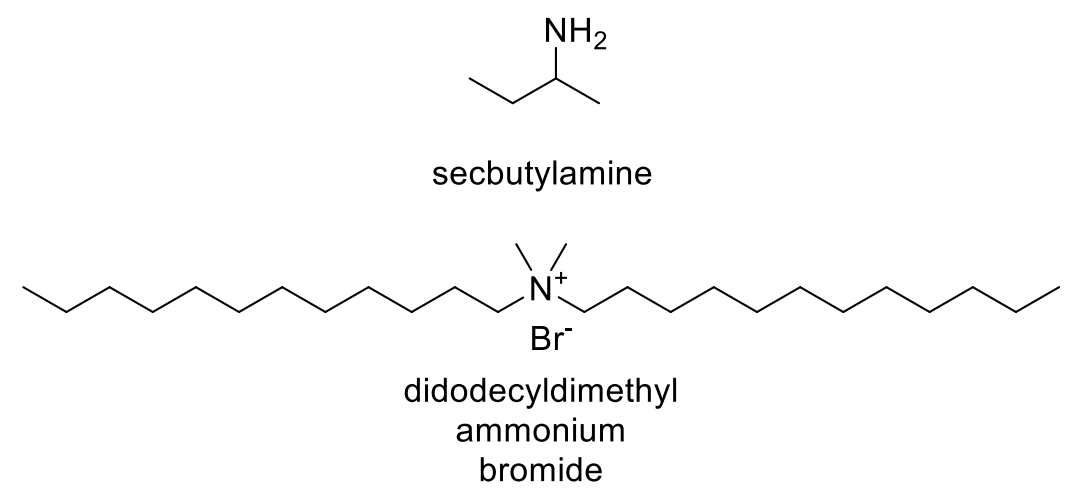

Chart IV-1. Sec-butylamine and didodecyldimethylammonium bromide (dDDdMABr), two of the stabilizing additives used to suspend 3-MPA capped PbS QDs in volatile organic solvents.

Despite these initial successes in obtaining a suspension of 3-MPA capped PbS QDs in a volatile organic solvent a decision was made to shift the stabilizing additive to a quaternary amine, didodecyldimethylammonium bromide (dDDdMABr, shown in Chart IV-1). The motivations for this change were: complexation of sec-butyl amine and 3MPA resulted in a highly viscous ionic liquid that was itself difficult to remove from deposited films of QDs, quaternary ammoniums have $\mathrm{pH}$ independent charging and would thus simplify complications of acid-base interactions between 3-MPA and the stabilizer, $\mathrm{dDDdMABr}$ is a non-volatile solid but has been demonstrated to be thermally degradable in QD films without destroying ordered QD arrays, ${ }^{137}$ while amines are well known QD ligands quaternary ammoniums are not expected to complex QD surfaces. 


\section{3) Spatially resolved multi-dimensional NMR}

Multi-dimensional NMR can be utilized to identify which species in solution participate at the surface of QDs. Both diffusion-ordered NMR spectroscopy (DOSY) and nuclear Overhauser effect spectroscopy (NOESY) have been used to great effect to identify ligands in complex solutions where multiple possible ligands are present. ${ }^{103,106,138}$ Both techniques take advantage of the fact that molecules associated with QDs have motional parameters similar to that of large particles instead of those of free molecular species. While DOSY resolves NMR signal by diffusion coefficient, it offers a weighted average signal in the diffusion dimension if molecules are in fast chemical exchange on the NMR time scale. For dynamically exchanging ligand shells where ligand species spend residency both on the QD surface and free in solution DOSY can provide difficult to interpret results when other factors may influence diffusion speed such as molecular complexation in acid-base reactions or ionic interactions.

2-dimensional NOESY is a through-space polarization cross-relaxation transfer NMR technique that utilizes the differences in rotational correlation time, ${ }^{\text {ii }} \tau_{c}$, between species in solution. In general, small species rotate more quickly in solution than large species while large particles rotate slowly. NOESY takes advantage of the fact that molecules rotating more quickly than the critical rotational correlation time of a given spectrometer, $\tau_{c}^{\text {crit }}$, generate weak and positively signed cross-peaks while molecules rotating more slowly generate strong and negatively signed cross-peaks. ${ }^{139}$ Molecules

\footnotetext{
ii Average time taken for a molecule to rotate 1 radian. ${ }^{139}$
} 
with $\tau_{c}$ near $\tau_{c}^{c r i t}$ generate null signal or weakly signed cross-peaks that are quickly lost in baseline noise. $\tau_{c}$ and $\tau_{c}^{c r i t}$ are defined in Equation IV-1 and Equation IV-2.

Equation IV-1. Critical rotational correlation time of a spectrometer of a given frequency, $\omega^{0}$.

$$
\tau_{c}^{c r i t}=\left|\frac{\sqrt{5}}{2 \omega^{0}}\right|
$$

Equation IV-2. Rotational correlation time for a small spherical particle experiencing isotropic rotation is given by the Stokes-Einstein relation. ${ }^{118} \eta$ is solvent microviscosity, $k_{b}$ is Boltzmann's constant.

$$
\tau_{c}=\frac{4 \pi \eta r^{3}}{3 k_{b} T}
$$

For QD based NMR applications this largely divides molecules of interest into two separate regimes; free ligands act like small molecules while bound ligands rotate at speeds similar to large particles.

$\tau_{c} \ll \tau_{c}^{c r i t}$, small free molecule behavior, weak positive signal

$\tau_{c} \gg \tau_{c}^{c r i t}$, large particle behavior (bound ligands), strong negative signal 


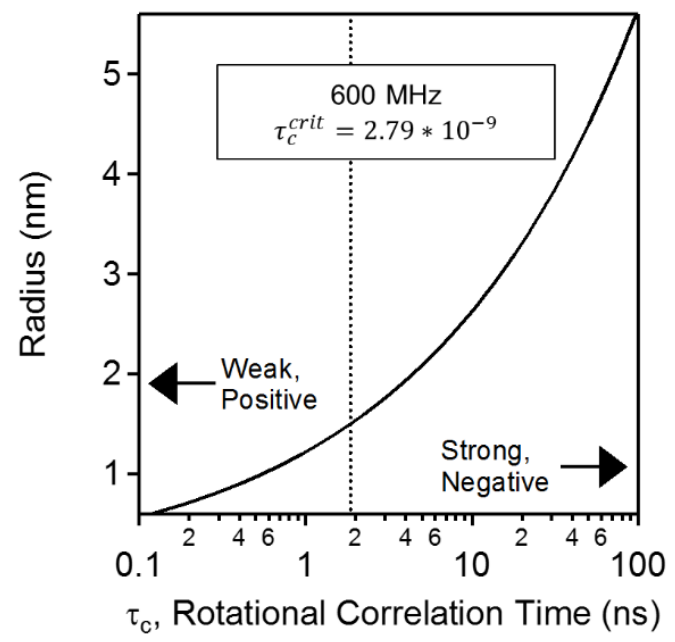

Figure IV-8. Rotational correlation time, $\tau_{c}$, vs radius via the Stokes-Einstein relation for small spherical objects. ${ }^{118}$ The critical rotational correlation time, $\tau_{c}^{\text {crit }}$, for a $600 \mathrm{MHz}$ NMR spectrometer is displayed as a vertical dashed line. Species rotating slower than this constant (larger $\tau_{c}$ ) generate strong negatively signed signal while species rotating faster $\left(\operatorname{smaller} \tau_{c}\right.$ ) generate weak positively signed signal.

This is advantageous in solutions where many ${ }^{1} \mathrm{H}$-containing species are present that can obfuscate small and broadened signal from ligands attached to the surface of QDs as only molecules that are attached to slowly rotating QDs generate strongly signed cross-peaks. The phenomenon of NOESY differentiating free $v s$ bound molecules with differently signed signal is demonstrated in Figure IV-9. 
A)<smiles>CCCCCC[CH]C=CCCCCCCC(=O)O</smiles>
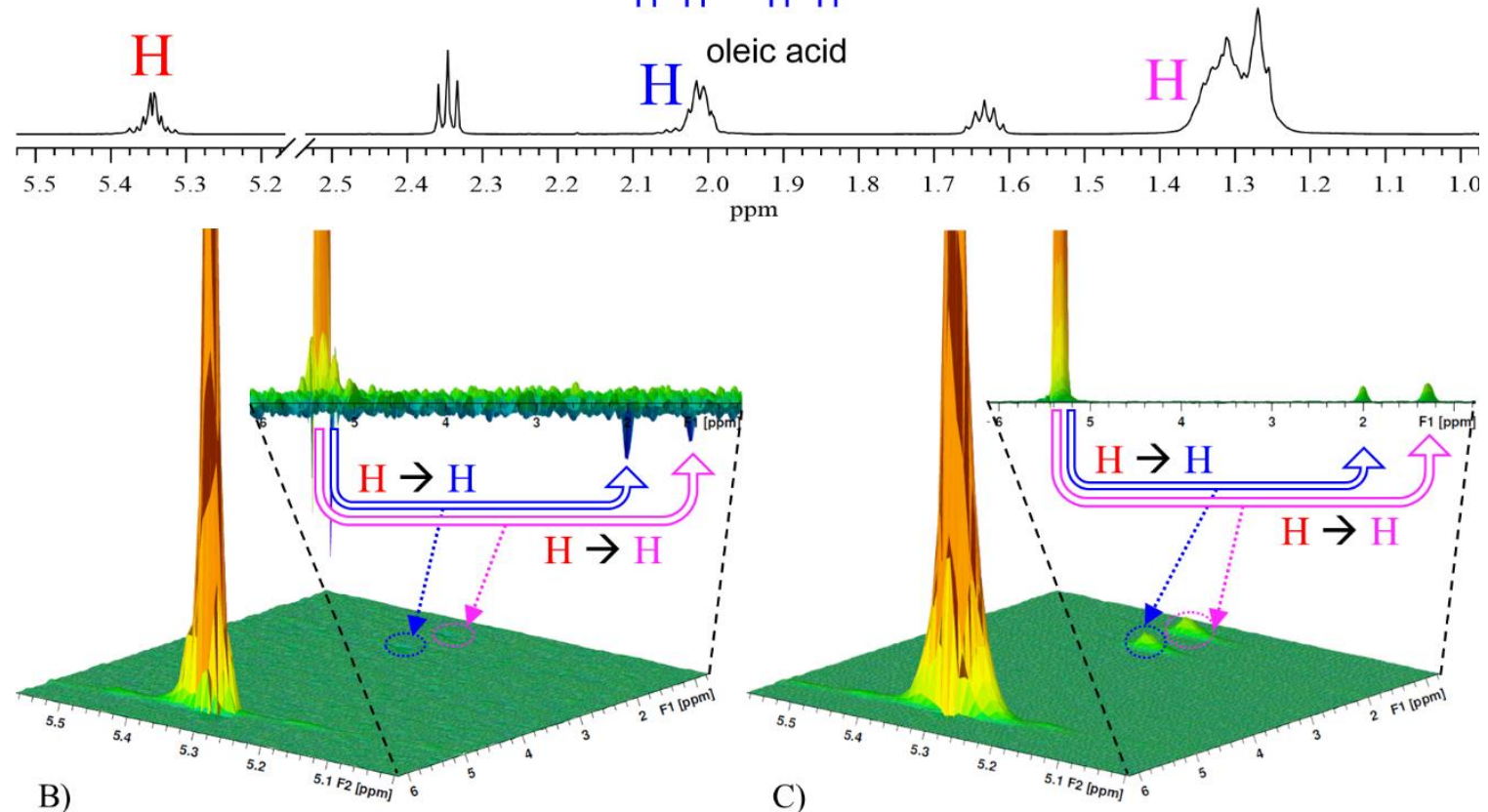

Figure IV-9. Projected 2-dimensional spectra showing differentiation of free $v s$ bound ligand signal signing in NOESY type experiments. A) The 1-dimensional ${ }^{1} \mathrm{H}$ spectrum of oleic acid. The polarization transfer of the alkene protons to the $\alpha$ - and $\beta$-alkene positions resolves as B) weakly positive (opposite sign to main diagonal alkene peak) when free in solution and C) strongly negative (same sign as main diagonal alkene peak) when present as oleate bound to the surface of PbS QDs. Both spectra have the same concentration of oleyl species.

\section{B) Abstract}

Colloidal 3-mercaptopropionic acid (3-MPA) capped Lead Sulfide quantum dots were prepared in a variety of organic solvents stabilized with a quaternary ammonium halide salt. The stabilized colloids' optical properties were studied through optical absorption and emission spectroscopy and found to be dependent on both the concentration of new ligand and stabilizer, and sample age. Nanocrystal ligand chemistry was studied through 
a combination of ${ }^{1} \mathrm{H}-\mathrm{NMR}$ and 2-dimensional Nuclear Overhauser Effect Spectroscopy (NOESY) which revealed full displacement of the original oleate ligand with a dynamically exchanging ligand shell. The colloids were studied optically and via NMR as they aged and revealed a quantitative conversion of monomeric 3-mercaptopropionic acid to its dimer, dithiodipropionic acid (dTdPA).

\section{C) Experimental Methods}

\section{1) Materials}

Oleic acid (OaH) (Technical Grade, Sigma, 112-80-1), bis(trimethylsilyl)sulfide (95\%, Sigma, 3385-94-2), 1-octadecene (Technical Grade, Sigma, 112-88-9), lead oxide (99.999\%, Sigma, 1317-36-8), anhydrous n-hexane (Alfa Aesar, 110-54-3), anhydrous toluene (99,8\%, Alfa Aesar, 108-88-3), anhydrous chloroform (EMD, 67-66-3), 1,4dichlorobenzene ( $\geq 99 \%$, Sigma, 106-46-7), didodecyldimethylammonium bromide (dDDdMABr, 99\%, Acros, 3282-73-3), dodecylamine (DDA, 98\%, Sigma, 124-22-1), sec-butylamine (99\%, Sigma, 13952-84-6), N,N-didodecylmethylamine (dDDMA, >85\%, TCI, 2915-90-4), 3,3'-dithiodipropionic acid (dTdPA, 99\%, Sigma, 1119-62-6), 3mercaptopropionic acid (3-MPA, 99\%, Alfa Aesar, 107-96-0), DMSO-D6 (99.9\% D, Sigma, 2206-27-1), $\mathrm{CDCl}_{3}(99.8 \%$ D, Acros. 865-49-6). All materials were used as received unless otherwise noted.

2) Synthesis of oleate capped $\mathrm{PbS}$ quantum dots $(\mathrm{Oa}-\mathrm{PbS})$

$\mathrm{PbS}$ quantum dots (QDs) were synthesized following a previously reported procedure, ${ }^{100}$ which was adapted from existing literature. ${ }^{38,74}$ Briefly, oleic acid (9.6 mmol, $\left.3.057 \mathrm{~mL}\right)$, 
1-octadecene (18 mL), and $\mathrm{PbO}$ (4 mmol, $0.8928 \mathrm{~g})$ were combined in a 3-neck flask in a glovebox (Mbraun Labstar). A separate flask containing 1-octadecene (10 mL) was also prepared in the glovebox. Both flasks were sealed and moved to a nitrogen filled Schlenk line outside the glovebox. The flask containing $\mathrm{PbO}$ was heated to $120^{\circ} \mathrm{C}$ and left stirring until it became clear. Bis(trimethylsilyl) sulfide $(2 \mathrm{mmol}, 0.421 \mathrm{~mL})$ was added under nitrogen to the flask containing only 1-octadecene. The bis(trimethylsilyl) sulfide solution was transferred rapidly to the stirred solution of $\mathrm{PbO}$ and oleic acid in 1octadecene and left for 1 minute before the heat source was removed. The freshly synthesized Oa-capped $\mathrm{PbS}(\mathrm{Oa}-\mathrm{PbS}) \mathrm{QDs}$ were precipitated with acetone in a $50 \mathrm{~mL}$ conical centrifuge tube and then centrifuged ( 5 minutes at 5000 RPM, Thermo Scientific Sorval ST16 Centrifuge with a Fiberlite F15 - 6x100y rotor) to separate the QDs from the reaction mixture. The supernatant was decanted and the QDs redispersed in toluene. A total of 4 precipitation/redispersion cycles were performed under ambient conditions. This was followed by a final precipitation cycle and transfer of the QD pellet into the glovebox. Inside the glovebox QDs were dispersed in anhydrous n-hexane to a concentration of $\sim 25 \mathrm{mg} \mathrm{mL}^{-1}$ and stored. Dilutions with n-hexane to $\sim 0.5 \mathrm{mg} / \mathrm{mL}$ were done inside the glovebox.

\section{3) Ligand Exchange}

$1 \mathrm{ml}$ of $\mathrm{Oa}-\mathrm{PbS}$ QDs $(0.5 \mathrm{mg} / \mathrm{mL})$ in $\mathrm{n}$-hexane were transferred to a glass vial and removed from the glovebox. The solvent was removed under vacuum, which yielded an Oa-PbS QD film. Same volume (1 mL) solutions of equimolar amounts of 3-MPA and dDDdMABr in $\mathrm{CHCl}_{3}$ were added to the vial to resuspend the QDs. The total molar 
amount of added 3-MPA and dDDdMABr was varied to prepare solutions having different molar ratios of added 3-MPA and dDDdMABr to initial molar amount of oleate $\left(\mathrm{Oa}^{-}\right)$. Initial molar amounts of $\mathrm{Oa}^{-}$(present as $\left.\mathrm{Oa}-\mathrm{PbS}\right)$ were quantified using nuclear magnetic resonance spectroscopy (see below).

\section{4) Nuclear Magnetic Resonance Spectroscopy}

${ }^{1} \mathrm{H}-\mathrm{NMR}$ spectra were acquired on a $600 \mathrm{MHz}$ Bruker Avance III with a $5 \mathrm{~mm}$ TXI probe using TopSpin 3.1. MestReNova was used to plot NMR spectra. Quantification of oleate was performed in triplicate for error analysis using $\mathrm{p}$-dichlorobenzene as an internal standard.

\section{5) Optical Spectroscopy}

Absorption spectra were acquired in reduced volume glass fluorimetry cells (Starna cells, 9F-Q-10-GL14-C) using a $4 \mathrm{~mm}$ path length. Absorption spectra were acquired using a Shimadzu 3600 UV-Vis-NIR spectrophotometer in dual-beam configuration with a solvent blank and the provided UVProbe software. Luminescence spectra were acquired with a StellarNet Dwarf-Star InGaAs NIR spectrometer and the provided Spectrawiz software. Samples were held in the same cuvettes as used for absorption spectra and were excited using a $<40 \mathrm{~mW} 532 \mathrm{~nm}$ green laser. The InGaAs CCD detector was calibrated using an incandescent bulb and the assumption that it was a perfect black-body emitter with a temperature of $2800 \mathrm{~K} .^{111}$

\section{D) Results}




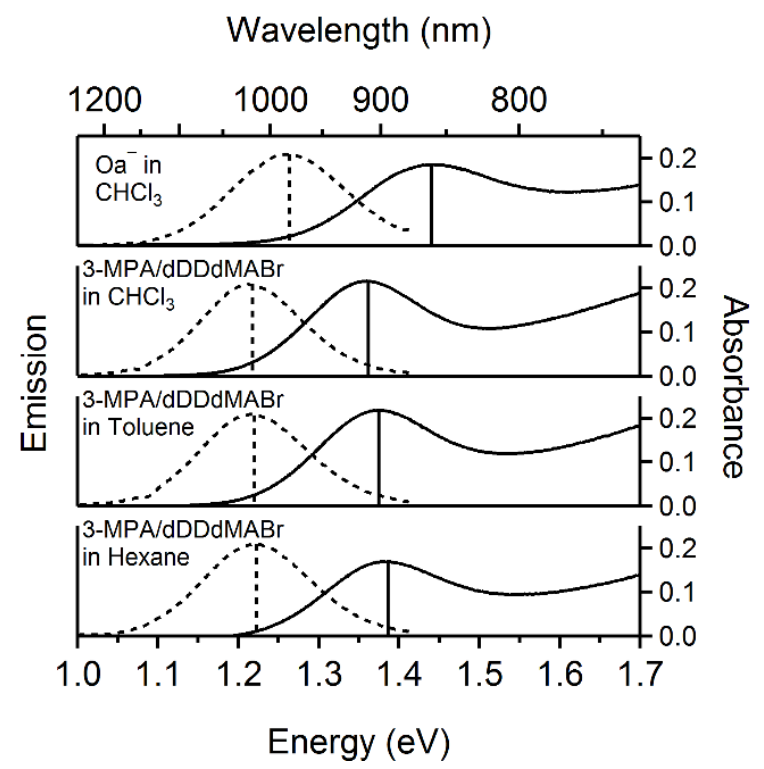

Figure IV-10. Absorption (solid) and emission (dashed) spectra of Oa-PbS QDs in CHCl3 and Oa-PbS QDs resuspended in various organic solvents with 3-MPA and dDDdMABr present in a 20:20:1 ratio of [3MPA]:[dDDdMABr]:[Oa-]. Vertical lines represent the fit position of the emission peak (dashed) and first excitonic absorption (solid) for each spectrum.

Figure IV-10 shows absorption and emission spectra of $\mathrm{Oa}-\mathrm{PbS}$ QDs in $\mathrm{CHCl}_{3}$ as well as dry $\mathrm{Oa}-\mathrm{PbS} \mathrm{QD}$ films resuspended in $\mathrm{CHCl}_{3}$, toluene, and hexane, all containing 3-MPA and dDDdMABr in a 20:20:1 ratio of [3-MPA]:[dDDdMABr]:[Oa $\left.{ }^{-}\right]$. Optical properties of the colloidal suspensions were only minimally dependent on the solvent used for resuspension. Absorption and emission peaks were red-shifted compared to Oa-PbS QDs, with larger apparent stokes-shifts for less polar solvents. Oa-capped PbS QDs had emission peaks centered around $1.26 \mathrm{eV}$. Emission peaks for exchanged colloids were centered around $1.22 \mathrm{eV}$ independent of solvent choice, while absorption peaks exhibited a minor dependence on solvent choice. Addition of only dDDdMABr produced colloidal suspensions, but resuspension to form colloidal suspensions were unsuccessful in the presence of 3-MPA without dDDdMABr (Figure S IV-1). 


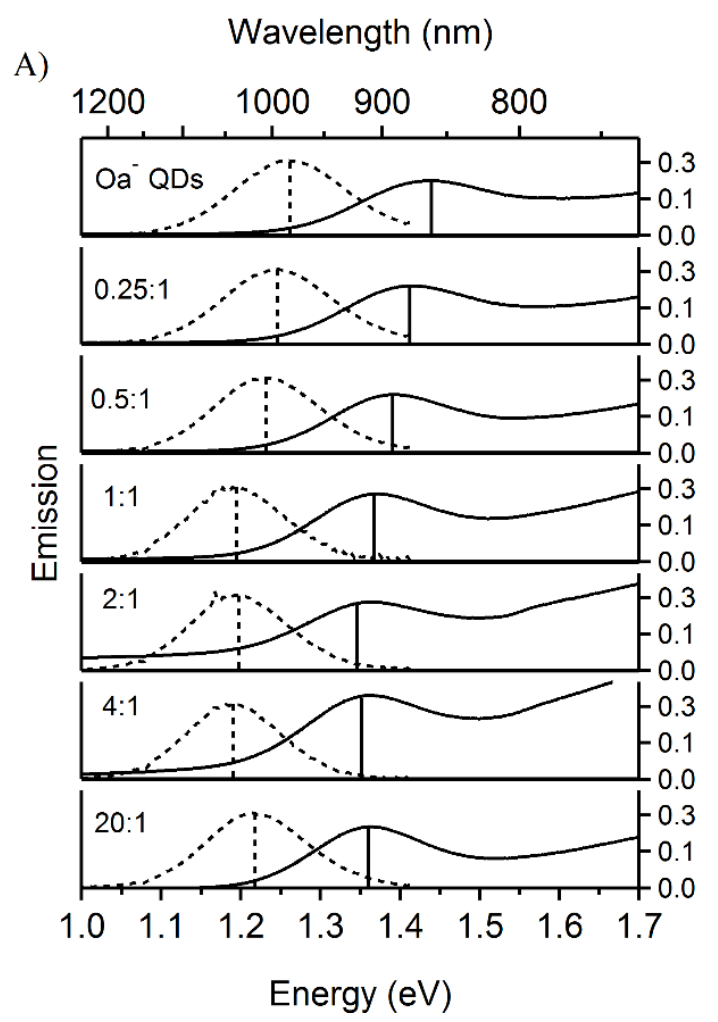

B)

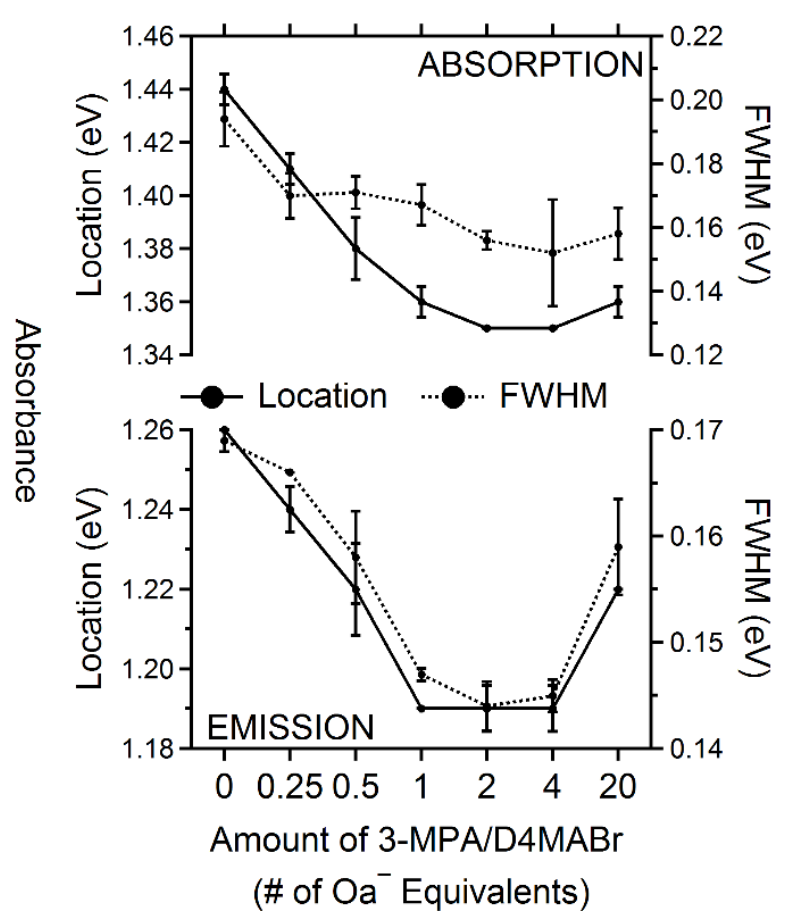

Figure IV-11. A) Representative absorption (solid) and emission (dashed) spectra from Oa-PbS QDs resuspended in solutions of equimolar amounts of 3-MPA and dDDdMABr in $\mathrm{CHCl}_{3} .0 .25: 1$ means 0.25 molar equivalents each of 3-MPA and dDDdMABr to 1 molar equivalent of $\mathrm{Oa}^{-}$, present originally as the QD ligand. Vertical lines represent the average fit position of the given peak from triplicate samples. B) Fitted peak location (black traces, left axis) and full width at half maximum (FWHM, dashed traces, right axis) from absorption (top) and emission (bottom) spectra of Oa-PbS QDs resuspended in solutions of equimolar amounts of 3-MPA and dDDdMABr in $\mathrm{CHCl}_{3}$, expressed as equivalents of $\mathrm{Oa}^{-}$originally present (bottom axis). All data points are the average of 3 separate experiments, and uncertainties are presented as one standard deviation. No error bars are displayed in cases where the fitted peak positions from separate experiments were identical within the limit of resolution $(0.01 \mathrm{eV})$.

Resuspension of $\mathrm{Oa}-\mathrm{PbS}$ QDs in $\mathrm{CHCl}_{3}$ was studied in detail. Figure IV-11 shows the absorption and emission spectra of dry films of Oa-PbS QDs resuspended with solutions of varying concentrations of 3-MPA and $\mathrm{dDDdMABr}$ in $\mathrm{CHCl}_{3}$. Concentrations are 
expressed in relation to oleate ([Oa- $]$ ) present in the sample, originally as the QD ligand. A wide range of concentrations of 3-MPA and dDDdMABr yielded colloidal solutions and in all cases well-resolved excitonic absorption peak and primary emission peak were preserved while colloids were suspended. Resuspension consistently resulted in a redshift of both the lowest-energy exciton absorption and emission peaks compared to OaPbS QDs. The amount of red-shift was dependent on the molar ratios of 3-MPA and dDDdMABr to $\mathrm{Oa}^{-}$. However, observed Stokes shifts were not strongly dependent on the molar ratios of 3-MPA and dDDdMABr to $\mathrm{Oa}^{-}$. Colloidal stability was also dependent on the ratio of [3-MPA] and [dDDdMABr] to $\left[\mathrm{Oa}^{-}\right]$. While all solutions initially resuspended the QDs successfully, solutions with ratios of [3-MPA and dDDdMABr]:[Oa $\left.{ }^{-}\right]$ from 1:1 to 4:1 precipitated within 15 minutes, forming visibly scattering solutions. Solutions with sub-stoichiometric ratios $(0.25: 1$ and $0.5: 1)$ and large excess (20:1) of [3MPA and dDDdMABr]:[Oa $\left.{ }^{-}\right]$remained in colloidal suspension for weeks.
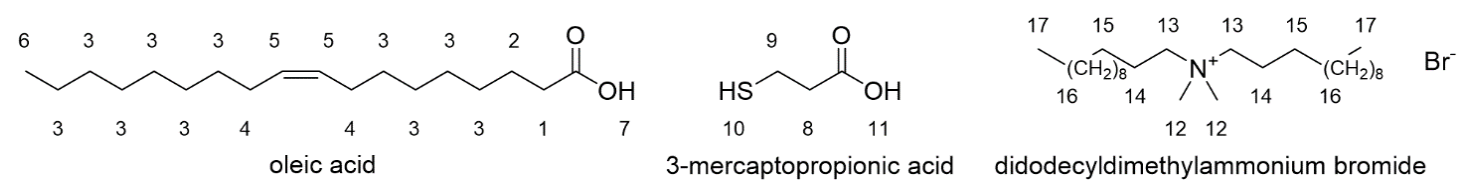

Chart IV-2. Oleic acid (OaH), 3-mercaptopropionic acid (3-MPA), and didodecyldimethylammonium bromide (dDDdMABr). Each molecule's distinguishable ${ }^{1} \mathrm{H}$ environments are labeled. 


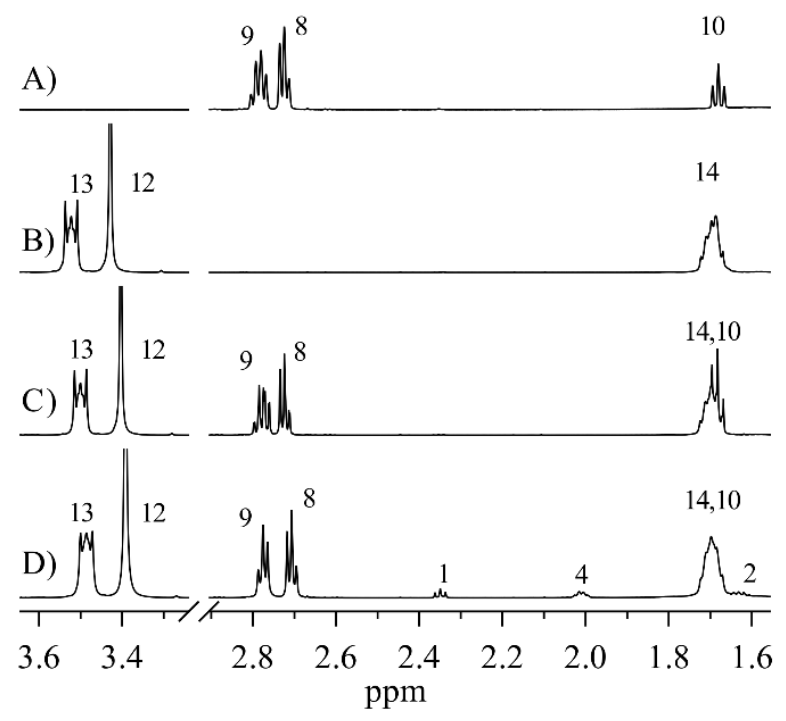

Figure IV-12. ${ }^{1} \mathrm{H}$ NMR spectra in $\mathrm{CDCl}_{3}$ of 3-MPA and dDDdMABr resonances. A) 3-MPA, B)

dDDdMABr, C) a 1:1 [3-MPA]:[dDDdMABr] solution, and D) an exchanged QD colloid with a 20:20:1 mixture of [3-MPA]:[dDDdMABr]:[Oa $\left.{ }^{-}\right]$.

Figure IV-12 shows ${ }^{1} \mathrm{H}$ NMR spectra of a dry film of Oa-PbS QDs resuspended with a 20:20:1 solution of 3-MPA and dDDdMABr in $\mathrm{CHCl}_{3}$. The spectral window covers 3 hydrogen resonances from 3-MPA (Chart 1); the thiol hydrogen resonance (10), $\alpha$-thiol hydrogen resonance (9), and $\beta$-thiol hydrogen resonance (8). In the absence of dDDdMABr and PbS QDs (Figure IV-12A) (10) was a well-defined triplet, (9) a quartet, and (8) a triplet. In the presence of dDDdMABr (Figure IV-12C) the peaks remained qualitatively the same but (10) was overlapping the $\beta$-ammonium peak from dDDdMABr (14); the resolution and peak splitting of resonances owing to dDDdMABr proximal to the amine center $(12,13$, and 14$)$ were not strongly affected by the presence of 3-MPA (Figure IV-12B and C). When 3-MPA was in the presence of both dDDdMABr and PbS 
QDs, the S-H resonance (10) was clearly suppressed, the $\alpha$-thiol resonance (9) switched from a resolved quartet to a triplet.

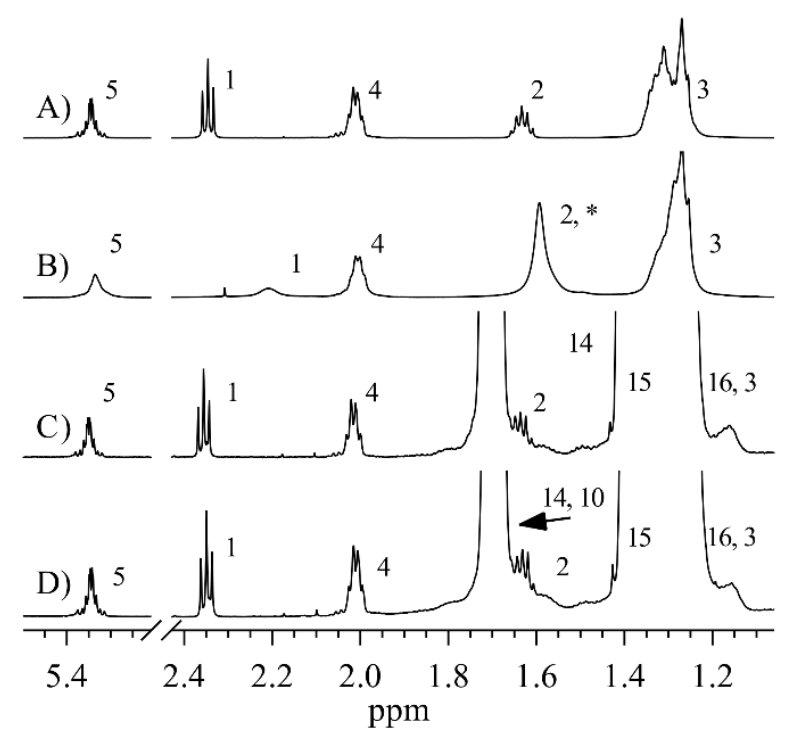

Figure IV-13. ${ }^{1} \mathrm{H}$ NMR spectra of oleyl resonances. A) OaH, B) Oa-PbS QDs, C) OaH with dDDdMABr in a 20:1 ratio of [dDDdMABr]:[OaH], and D) Oa-PbS QDs with 3-MPA/dDDdMABr added in a 20:20:1 ratio of [3-MPA]:[dDDdMABr]:[Oa- $]$. The concentration of $\mathrm{Oa}$ is the same in all spectra. A peak attributed to water is present in some samples and marked with " **”.

Figure IV-13 shows ${ }^{1} \mathrm{H}$ NMR spectra of a dry film of Oa-PbS QDs resuspended with a 20:20:1 solution of 3-MPA and dDDdMABr in $\mathrm{CHCl}_{3}$. The spectral window focuses on hydrogen resonances from oleyl species that present as 5 separate peaks; the $\alpha$ - and $\beta$ carboxylate hydrogen resonances ( 1 and 2), the $\alpha$-alkene hydrogen resonance (4), the alkene hydrogen resonance (5), and, excluding the carboxylate and terminal methyl hydrogen resonances, the remaining hydrogen resonances overlapping around $1.3 \mathrm{ppm}$ (3). Free $\mathrm{OaH}$ presented distinctly different peaks from $\mathrm{Oa}^{-}$bound to the surface of $\mathrm{PbS}$ QDs (Figure IV-13A and B), and the presence of dDDdMABr did not affect free $\mathrm{OaH}$ 
resonances (Figure 4C). Oleyl hydrogen resonances from Oa-PbS QDs resuspended with 3-MPA/dDDdMABr had splitting patterns and chemical shifts consistent with free OaH.
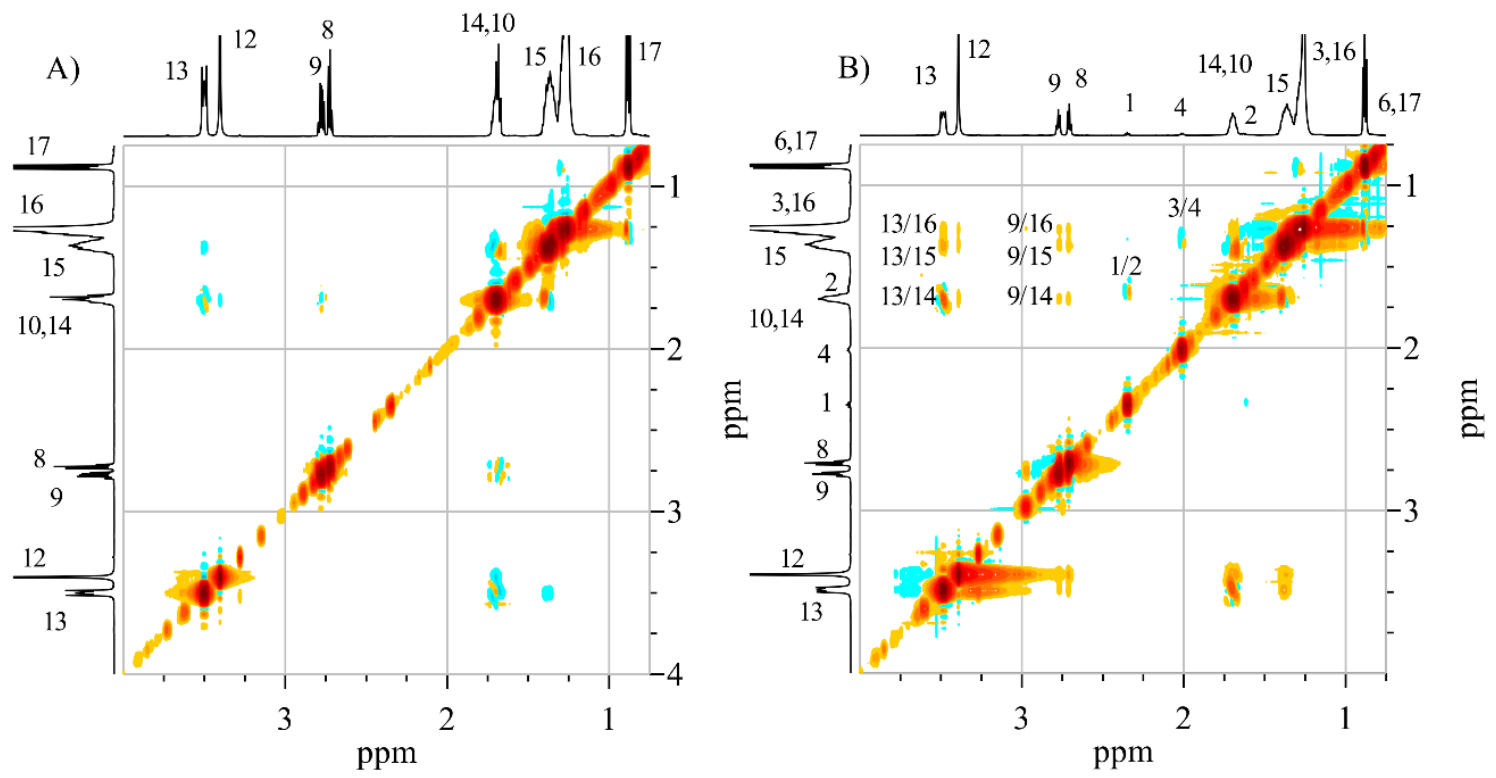

Figure IV-14. NOESY spectra demonstrating ligand interaction with PbS QDs. A) 3-MPA and dDDdMABr in a 1:1 [3-MPA]:[dDDdMABr] ratio in $\mathrm{CDCl} 3$ and $\mathrm{B}$ ) an exchanged QD colloid with a 20:20:1 mixture of [3-MPA]:[dDDdMABr]:[Oa- ]. The concentration of 3-MPA and dDDdMABr is the same in both spectra.

Figure IV-14 demonstrates the effect on NOESY spectra of adding 3-MPA and dDDdMABr to a suspension of Oa-PbS QDs. A 1:1 mixture of 3-MPA and dDDdMABr only built weak positive NOE cross peaks in the absence of QDs (Figure IV-14A). In the presence of QDs 3-MPA and dDDdMABr generated strong negative cross peaks both for hydrogens on the same molecule but separated by several C-C bonds (Figure S IV-2 e.g., 13/16), and for hydrogens on different chemical species (Figure IV-14B, e.g., 9/16).

2-dimensional $\mathrm{NOE}$ spectra from solutions of free $\mathrm{OaH}$ in $\mathrm{CDCl}_{3}$ and a colloidal suspension of $\mathrm{Oa}-\mathrm{PbS} \mathrm{QDs}$ in $\mathrm{CDCl}_{3}$ were different from each other. Free $\mathrm{OaH}$ showed 
weak positive or antiphase cross peaks (Figure S IV-2, e.g., 3/5, 4/5 cross peaks). Oa-PbS QDs under the same conditions and total oleyl concentration showed strong negative cross peaks consistent with surface bound oleate species (Figure S IV-2). Oa-PbS QDs resuspended with 3-MPA/dDDdMABr showed weak antiphase cross-peaks (Figure IV-14B 1/2, 3/4 and Figure S IV-3 3/5, 4/5).

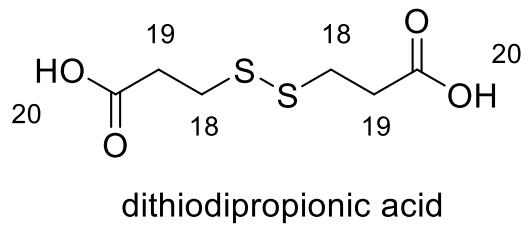

Chart IV-3. Dithiodipropionic acid (dTdPA) with distinguishable proton environments labeled.
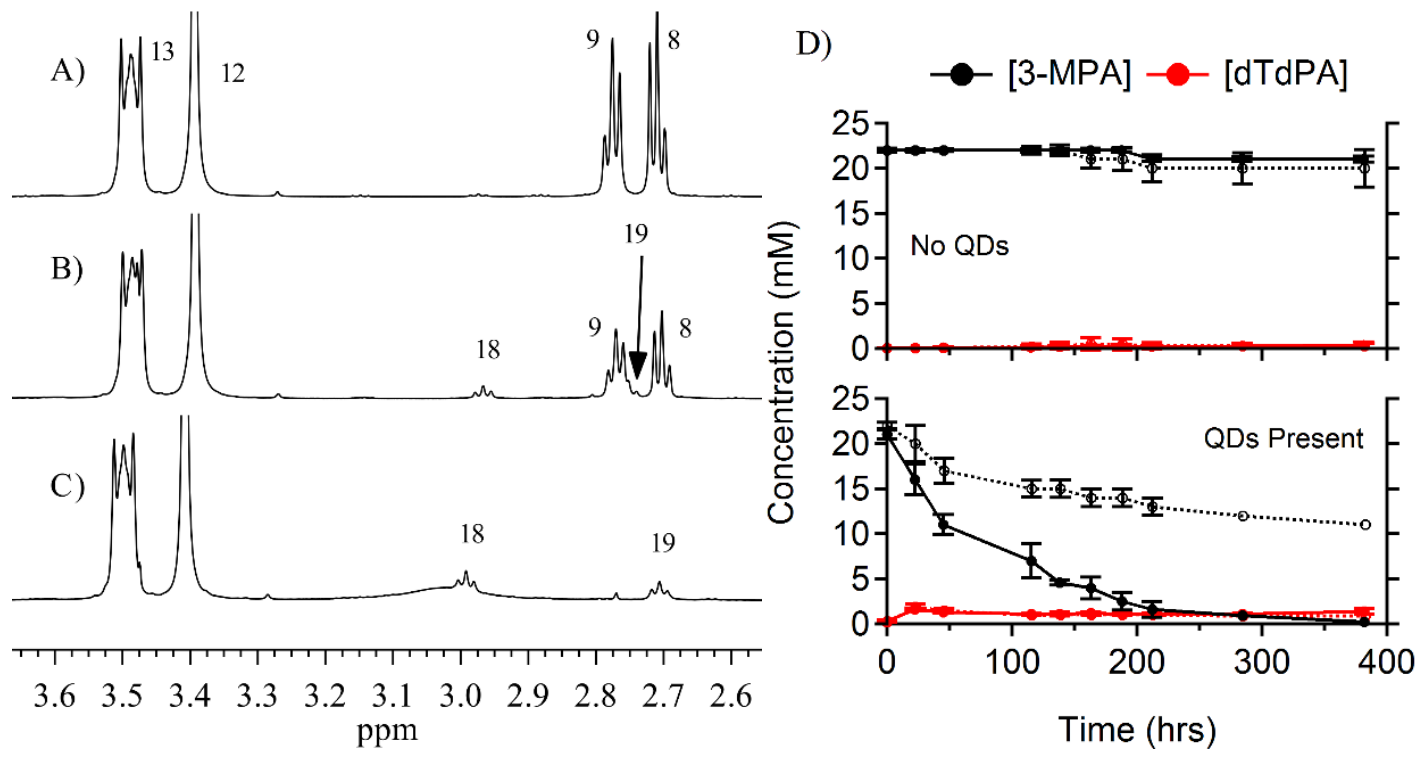

Figure IV-15. ${ }^{1} \mathrm{H}$ NMR spectra and quantification of dTdPA formation from colloids of Oa-PbS QDs with 3-MPA/dDDdMABr added in a 20:20:1 ratio of [3-MPA]:[dDDdMABr]:[Oa-] in $\mathrm{CDCl}_{3}$. Samples were kept in the dark (dotted lines) or under ambient light (solid lines) and allowed to age for 16 days.

Representative spectra of A) a freshly prepared sample, B) a sample stored in the dark for 16 days, C) a freshly prepared sample subsequently stored in ambient light for 16 days, D) Concentrations of 3-MPA and 
dTdPA of samples stored in different light conditions throughout the aging process were determined by comparing integrals from 3-MPA ( $9 \& 8$ ) to the well resolved singlet $\alpha$-amine of dDDdMABr (12).

3-MPA/dDDdMABr stabilized PbS QDs facilitated the conversion of 3-MPA to dithiodipropionic acid (dTdPA) (Figure IV-15 and Figure S IV-4). In the absence of PbS QDs approximately 10\% of all available 3-MPA was depleted from the solution and small amounts of dTdPA were observed (Figure S IV-5) over the course of 16 days. In the presence of PbS QDs, the concentration of 3-MPA decreased significantly. In the dark, the concentration of 3-MPA had decreased to less than half of the original concentration after 16 days. Under ambient illumination almost all 3-MPA had been removed from the solution within 9 days. The decrease in the concentration of 3-MPA was correlated to an increase in the concentration of dTdPA. However, the concentration reached a maximum and the solution became saturated with dTdPA within 50 hours of aging in the presence of $\mathrm{PbS}$ QDs and under ambient illumination. This was concurrent with the formation of crystals (Figure S IV-6). The crystals were removed from solution, and found to be dTdPA via NMR analysis (Figure S IV-7); by NMR, no other species were detected in the re-dissolved crystalline sample. The QDs remained in colloidal suspension for the duration of the experiment, but absorption and emission peaks were observed to shift, and trend with the concentration of 3-MPA (Figure S IV-8). A marked blue shift was observed over the first 50 hours but peak positions stabilized after the first 50 hours. 


\section{E) Discussion}

\section{1) Optical Properties}

PbS QDs originally capped with oleate were resuspended with solutions of 3-MPA and dDDdMABr in $\mathrm{CHCl}_{3}$, toluene, and hexane. Absorption and emission peaks shifted slightly upon resuspension. The magnitude of the excitonic peak position redshift for stable suspensions with 20:20:1 [3-MPA]:[dDDdMABr]:[Oa ${ }^{-}$] was similar to previously observed red-shifting seen during bi-phasic ligand exchange to form 3-MPA-capped $\mathrm{PbS}$ QDs in DMSO. ${ }^{100}$ Oa-capped PbS QDs had emission peaks centered around $1.26 \mathrm{eV}$. Emission peaks for exchanged colloids were centered around $1.22 \mathrm{eV}$ independent of solvent choice, while absorption peaks exhibited a minor dependence on solvent choice. Solvatochromic effects of similar magnitude have previously been observed for dispersed QDs in solvents of varying dielectric constants. ${ }^{28}$

Ligand exchange to form colloidally stable solutions was not possible in $\mathrm{CHCl}_{3}$, toluene, or hexane using only 3-MPA. The addition of 3-MPA alone resulted in immediate agglomeration to form a clear supernatant solution within minutes. Despite rapid agglomeration, it was possible to obtain emission spectra within the first minute that revealed a more extensive red-shift than combined 3-MPA/dDDdMABr solutions (Figure S IV-1). Extended red-shifting of optical spectra has previously been observed as a result of enhanced particle-particle coupling in QD solids, ${ }^{87,91}$ and is consistent with increased coupling due to agglomeration. This was further supported by the loss of any clearly resolvable exciton peak in the absorption spectra. The observed emission intensity of 3-MPA only suspensions was $\sim 12 \%$ of suspensions with 3-MPA and dDDdMABr 
together. The addition of dDDdMABr by itself induced a minor red-shift in both the absorption and emission peak positions with the QDs remaining in colloidal suspension. This is consistent with previously observed spectral changes with PbS QDs that have undergone ligand exchange from organic surfactants such as oleate to a halide shell. ${ }^{59,140-}$ 142

Exchanged QDs were allowed to age while following changes in optical properties (Figure S IV-8). A blue-shift over time was observed as the colloids aged; this shift roughly followed the depletion of 3-MPA in the colloids observed during NMR aging studies (Figure IV-15). However, the suspensions maintained well-defined emission and absorption peaks throughout extended aging and the nanocrystals remained in suspension. Small amounts of light scattering were observed as crystals formed inside the solutions due to photo-enhanced catalytic conversion from monomer 3-MPA to dimer dTdPA (Figure S IV-6).

\section{2) Ligand Chemistry}

Both one-dimensional and two-dimensional NMR has been used to successfully identify ligands on the surface of nanocrystals by taking advantage of differences in chemical shifts and the rotational correlation time between free molecular species and those bound to a nanocrystal surface. ${ }^{40,103,115,118,143}$ This approach was used to further investigate the ligand chemistry resulting from exposure of $\mathrm{Oa}-\mathrm{PbS}$ QDs to 3-MPA/dDDdMABr solutions. 


\section{3) Resuspension Gave 3-MPA Stabilized PbS QDs}

3-MPA replaced surface-bound $\mathrm{Oa}^{-}$to give $\mathrm{OaH}$ when $\mathrm{Oa}-\mathrm{PbS} \mathrm{QD}$ films were resuspended with 3-MPA and dDDdMABr in $\mathrm{CHCl}_{3}$. Specifically, the data suggest that 3-MPA interacted with the PbS QDs via the thiol moiety in a dynamic fashion, and that dDDdMA ${ }^{+}$associated with the 3-MPA-capped PbS QDs. ${ }^{1} \mathrm{H}-\mathrm{NMR}$ (Figure IV-12D) showed loss of the 3-MPA thiol protons, and, consistent with this, the $\alpha$-thiol protons (9) changed from a quartet to a triplet. NOE spectra showed that both 3-MPA and dDDdMABr spent time in close proximity to one another while associated with the QD surface (Figure IV-14). Both 3-MPA and dDDdMABr generated strong negative NOE cross-peaks consistent with attachment to a QD surface and significant cross-relaxation between 3-MPA and dDDdMABr was observed (Figure IV-14). This is consistent with previous observations of dynamically capped 3-MPA-PbS QDs in DMSO. ${ }^{100}$

dDDdMABr by itself did not cause the above-described spectral changes. When 3MPA and dDDdMABr were mixed in a 1:1 ratio (Figure IV-12C) there were no significant changes to the observed splitting patterns of 3-MPA. The normalized integration of the convoluted peak combining resonances 10 (thiol of 3-MPA, one proton) and 14 ( $\beta$-amino of $\mathrm{dDDdMA}^{+}, 4$ protons due to symmetry) sum to their proper value representing 5 protons. The $\mathrm{COOH}$ resonance of 3-MPA (11) shifted, broadened, and its integral was reduced to approximately half (Figure S IV-10) compared to 3-MPA alone, typical behavior for a $\mathrm{COOH}$ peak in exchange. Figure IV-14 shows NOE spectra

demonstrating that a 1:1 solution of 3-MPA/dDDdMABr did not generate negative cross 
peaks associated with slow tumbling molecules or have significant through space intermolecular cross-relaxation in solution when QDs were not present.

\section{4) Ligand Exchange Released Free $\mathrm{OaH}$}

QD surface bound $\mathrm{Oa}^{-}$was released to yield free oleic acid $(\mathrm{OaH})$ in an overall X-type ligand exchange. ${ }^{114}$ Figure S IV-2 demonstrates the differences between free $\mathrm{OaH}$ in solution and $\mathrm{Oa}^{-}$bound to the surface of PbS QDs. Most notably for bound vs. free $\mathrm{Oa}^{-}$, the alkene peak near $5.4 \mathrm{ppm}$ (5) became less resolved and shifted upfield, and the $\alpha$ carboxylate peak (1) was significantly broadened and shifted upfield. Figure IV-12A and D reveal that oleyl resonances had the same splitting patterns, broadening, and chemical shift as free $\mathrm{OaH}$ when $\mathrm{Oa}-\mathrm{PbS}$ QD films were resuspended with 3-MPA and dDDdMABr in $\mathrm{CHCl}_{3}$. This indicates that $\mathrm{Oa}^{-}$was completely displaced from the QD surface. NOE spectra show that oleyl species released into solution as a free species and did not associate strongly with the QD after ligand exchange (Figure S IV-2). Free oleyl species generated either weak positive NOE buildup or anti-phase peaks in cases where scalar coupling was present (e.g., cross-peaks 1/2,3/4). In contrast, $\mathrm{Oa}^{-}$bound to the surface of PbS QDs generated strong negative NOE cross-peaks. The same observations have been made previously for $\mathrm{Oa}^{-}$bound to $\mathrm{PbSe}$ QDs wherein only bound ligands built up a strong negative NOE signal. ${ }^{118}$ After ligand exchange, strong negative NOE cross peaks were absent, and in agreement with NOESY data for free oleyl species (c.f. 3/4 and 1/2 cross peaks in Figure IV-14 and Figure S IV-2).

The identity of the released form of oleyl species was likely the free acid form, OaH; the chemical shifts associated with oleate complexation with amines (to form 
carboxylate/ammonium ion pairs) seen in Figure S IV-11 were not observed when Oa$\mathrm{PbS}$ QDs was re-suspended with 3-MPA/dDDdMABr in $\mathrm{CHCl}_{3}$. Instead, the oleyl resonances were identical to those of $\mathrm{OaH}$, which did not have significant interaction with dDDdMABr on its own (Figure IV-13C); when dDDdMABr and OaH were mixed in a 20:1 ratio of [dDDdMABr]:[OaH] Figure S IV-9C) the OaH resonances appeared identical to that of free $\mathrm{OaH}$.

\section{5) dDDdMABr displaces oleate from $\mathrm{Oa-PbS}$ when 3-MPA is not present}

dDDdMABr facilitated the overall 3-MPA for $\mathrm{Oa}^{-}$ligand exchange, and by itself interacted with Oa-PbS QDs. One-dimensional ${ }^{1} \mathrm{H}-\mathrm{NMR}$ spectroscopy of a 20:1 ratio of [dDDdMABr]:[OaH] $\left(\right.$ or $\left.\mathrm{Oa}^{-}\right)$further explored changes to $\mathrm{Oa}^{-}$resonances as a result of interaction between $\mathrm{OaH}$ and dDDdMABr in solution (Figure S IV-9). In samples without dDDdMABr the $\alpha-\mathrm{COOH}$ protons (1), $\beta-\mathrm{COOH}$ protons (2), and alkene protons (5) of oleyl species were severely broadened when attached to PbS QDs (Figure S IV-9B) but had well resolved splitting when present as free OaH (Figure S IV-9A). In contrast to this, Figure S IV-9D shows that the dDDdMABr in a 20:1 ratio of [dDDdMABr]:[Oa ${ }^{-}$] affects the broadening and changes the chemical shift of $\mathrm{Oa}^{-}$resonances when in the presence of $\mathrm{Oa}-\mathrm{PbS}$ QDs such that the signal does not fit that of either free $\mathrm{OaH} / \mathrm{Oa}^{-}$or $\mathrm{Oa}^{-}$bound at the surface of PbS QDs. When $\mathrm{dDDdMA}^{+}$was introduced to suspensions of Oa-PbS QDs at a 20:1 ratio of [dDDdMABr]:[Oaa $]$ the strong negative cross-peaks $\mathrm{Oa}^{-}$ demonstrated when on the surface of PbS QDs (Figure S IV-2) were not observed. Instead, the cross peak of $\mathrm{Oa}^{-}$belonging to transfer between resonances 4/5 (strongly negative in suspensions of $\mathrm{Oa}-\mathrm{PbS} \mathrm{QDs}$ ) resolved as an antiphase peak while the $3 / 5$ 
cross-peak was not resolved. This indicates that the strong Oa-QD bond was disrupted by the addition of dDDdMABr. Figure S IV-12 shows that dDDdMA ${ }^{+}$built up weakly positive or scalar-coupled antiphase cross-peaks when alone in solution and when in the presence of PbS QDs. This indicates that $\mathrm{dDDdMA}^{+}$did not directly associate with the PbS QDs like a bound molecular species, and that oleyl species were neither freely diffusing or bound to $\mathrm{PbS}$ QDs after exposure to dDDdMABr.

Halides, including bromide, have been shown to act as QD ligands. ${ }^{59,140-142}$ Bromide may interact with Oa-QDs, and would not be observable in ${ }^{1} \mathrm{H}$ NOESY spectra as a bound ligand. $\mathrm{Br}^{-}$can be considered an anionic X-type ligand. ${ }^{114}$ Typically a proton would be required to release $\mathrm{OaH},{ }^{107}$ but the formation of ion pairs is an alternate explanation that has support in the literature. ${ }^{135} \mathrm{dDDdMA}^{+}$stands as an alternative counter-ion to balance the release of $\mathrm{Oa}^{-}$upon exchange with $\mathrm{Br}^{-}$at the surface of the QD, and vesicle formation between $\mathrm{OaH}$ and dDDdMABr has previously been observed. ${ }^{144}$ Hence, we explored the hypothesis that oleate was displaced to yield either free oleyl species, or as a lead oleate complex.

Mixtures of $\mathrm{OaH}$ and either didodecylmethylamine (dDDMA) or dodecylamine (DDA), to form $\mathrm{Oa}^{-} / \mathrm{dDDMAH}^{+}$or $\mathrm{Oa}^{-} / \mathrm{DDAH}^{+}$were used to probe the release of $\mathrm{Oa}^{-}$ from Oa-PbS QDs as an ion pair with $\mathrm{dDDdMA}^{+}$(Figure S IV-11) by examining changes to oleyl resonances in the presence of amines. The formation of $\mathrm{Oa}^{-} / \mathrm{dDDMAH}^{+}$and $\mathrm{Oa}^{-} / \mathrm{DDAH}^{+}$strongly affected the broadening and chemical shift of the $\alpha-\mathrm{COOH}(1), \beta-$ $\mathrm{COOH}(2)$, and alkene (5) resonances of oleyl species. However, there was not sufficient overlap of these spectra to assign such a complex as the origin of the signal observed for 
oleyl species when dDDdMABr was mixed with Oa-PbS QDs in the absence of 3-MPA (Figure S IV-9D).

Mixtures of $\mathrm{Pb}(\mathrm{Oa})_{2}$ and $\mathrm{dDDdMABr}$ were used to probe whether QD-bound oleate was released as a lead oleate complex. Metal carboxylate displacement has been observed due to L-type ligand assisted desorption in metal chalcogenide QDs. ${ }^{22}$ While there are no L-type ligands present in combinations of dDDdMABr and Oa-PbS QDs, we combined $\mathrm{Pb}(\mathrm{Oa})_{2}$ with $\mathrm{dDDdMABr}$ to probe the possibility of this as a mechanism of displacement as oleyl species were clearly not attached to the QD surface (Figure S IV-9F-G). A combination of $\mathrm{Pb}(\mathrm{Oa})_{2}$ and dDDdMABr in a 20:1 ratio of [dDDdMABr]:[Oa $\left.{ }^{-}\right]$showed oleyl signal remarkably close to that of Oa-PbS QDs with dDDdMABr (Figure S IV-9D) but is not identical. Computational surface studies have indicated that the surface of Oa$\mathrm{PbS}$ QDs is substantially different than that of $\mathrm{Cd}$ analogues in that only one $\mathrm{Oa}^{-}$ molecule per excess $\mathrm{Pb}^{2+}$ atom is present on the surface of the QDs. ${ }^{21}$ Other quantitative studies of lead chalcogenide QDs have also found a 1:1 ratio between excess (nonstoichiometric to chalcogen atoms) lead atoms and surface bound oleate. ${ }^{104}$ As surface bound $\mathrm{OH}^{-}$has been shown to also be present, it may be that $\left[\mathrm{Pb}\left(\mathrm{Oa}^{-}\right)\left(\mathrm{OH}^{-}\right)\right]$was released.

In solutions where 3-MPA is present proton transfer from 3-MPA to $\mathrm{Oa}^{-}$complexed with $\mathrm{dDDdMA}^{+}$(forming a 3-MPA$/ \mathrm{dDDdMA}^{+}$complex) is still a viable route for the incorporation of $\mathrm{Br}^{-}$into the QD ligand shell. This is not considered problematic for the formation of QD “inks”, as the addition of halides to the ligand shell of QDs has been 
shown to be an effective means of minimizing shallow trap states deleterious to QD photovoltaic device performance and has become a routine synthetic procedure.

\section{6) Co-stabilization is required for 3-MPA capped PbS QDs in non-polar solvents} Addition of 3-MPA without dDDdMABr present led to immediate precipitation of the QDs while addition of 3-MPA with dDDdMABr present allowed for extended colloidal suspension. Similar behavior requiring co-stabilization has been observed in $\mathrm{HfO}_{2}$ and $\mathrm{ZrO}_{2}$ nanocrystals, where both a carboxylic acid and an alkylamine were required to be present to support colloidal suspension of the nanocrystals in non-polar media. ${ }^{105}$ In that report, the alkylamine was determined to act as a Brønsted base causing deprotonation of the carboxylic acid, allowing it to replace a dissociated Brønsted acid already present on the natively synthesized nanocrystals. Subsequent purification steps allowed isolation of the nanocrystals without the presence of the alkylamine. A key difference in this report is the lack of obvious acid/base reactivity of the quaternary ammonium salt dDDdMABr. $\mathrm{PbS}$ QDs have also been demonstrated to be stabilized by arenethiolates mixed with amines ${ }^{86,87}$ In those reports the replacement of native $\mathrm{Oa}^{-}$was attributed to the increased acidity of arenethiols relative to alkylthiols that is enhanced further in the presence of an amine. The use of a quaternary amine in this report rules out the possibility of such complicated acid-base interactions, though we do note that stabilization of PbS QDs with 3-MPA and simple amines is also possible. The re-suspension of Oa-PbS QDs with 3MPA and sec-butylamine (Figure S IV-13) gave similar shifts in optical spectra as observed with dDDdMABr as a supporting co-stabilizer. In this case, the stability of the colloid was found to be dependent on the concentration of amine. With a 20:20:1 ratio of 
[secBA]:[3-MPA]:[Oa $\left.{ }^{-}\right]$the colloid was mildly aggregated. With a 40:20:1 ratio of [secBA]:[3-MPA]:[Oa $\left.{ }^{-}\right]$the colloid was fully stabilized with no scattering artifacts in the absorption spectra.

\section{7) 3-MPA to $d T d P A$ Catalysis}

The conversion of thiols to dithiols is an important chemical transformation used to, for example, remove thiols from petroleum fractions. ${ }^{145}$ It was previously observed that $3-$ MPA-PbS QDs suspended in DMSO catalyzed the conversion of 3-MPA to its dimer, 3,3'-dithiodipropionic acid (dTdPA). ${ }^{100}$ DMSO was likely the oxidant. ${ }^{101,102}$ Thiols, especially those bearing carboxylic acid moieties including 3-MPA, have previously been observed to be photo-catalytically dimerized on the surface of CdSe QDs. ${ }^{146}$ In this study it was observed that after 24 hours small crystalline precipitates formed in samples of PbS QDs suspended with 3-MPA/dDDdMABr present and aged under ambient light. The solid was collected, re-suspended in DMSO- $\mathrm{D}_{6}$ and confirmed as $\mathrm{dTdPA}$ by spiking the sample with dTdPA. No special care was taken to exclude $\mathrm{O}_{2}$, which is a likely candidate as the oxidant. Colloidal suspensions of PbS QDs with ratios of 20:20:1 [3MPA]:[dDDdMABr]:[Oa $\left.{ }^{-}\right]$were allowed to age under dark and ambient light conditions while collecting NMR spectra to observe formation of dTdPA further. Figure IV-15A shows a representative spectrum prior to any aging occurring. After aging in the dark there is significant depletion of resonances 9 and 10 from loss of 3-MPA due to dimer conversion while resonances 18 and 19 did not increase in intensity further after $\sim 24$ hours due to saturating the sample in $\mathrm{CDCl}_{3}$. Figure IV-15B shows the dependence of the conversion of 3-MPA to dTdPA on both light conditions and the presence of QDs. With 
no QDs present, there was no statistically relevant difference in the conversion rate of 3MPA to dTdPA. With QDs present the conversion rate was significantly increased and dependent on illumination. The conversion of 3-MPA to dTdPA trended similarly to the blue-shift in optical spectra as the colloid aged (Figure S IV-8). This is consistent with the reversal of the ligand induced red-shift upon ligand exchange with 3-MPA. The high purity of collected dTdPA and reasonable conversion rate suggests that the system warrants further investigation.

\section{F) Conclusion}

Didodecyldimethylammonium bromide stabilized colloidal 3-MPA capped PbS QDs were prepared in $\mathrm{CHCl}_{3}$, toluene, and hexane. 1-D ${ }^{1} \mathrm{H}-\mathrm{NMR}$ and 2-D NOE spectra were consistent with complete displacement of the original oleate ligand as free oleyl species (either oleic acid or oleate), likely forming oleic acid in an overall X-type ligand exchange by proton transfer from 3-MPA. 1-D ${ }^{1} \mathrm{H}-\mathrm{NMR}$ and 2-D NOE spectra were consistent with 3-MPA dynamically exchanging between being surface bound and free in solution. This is similar to our previous literature report of 3-MPA capped PbS QDs in DMSO. ${ }^{100} \mathrm{dDDdMA}^{+}$associated with the QD surface in colloids containing both 3-MPA and dDDdMABr, though it was ultimately unclear if it participates directly at the QD surface or is merely associated with 3-MPA bound to the surface of the QD. Separately, the 3-MPA capped PbS QDs were observed to photo-catalyze the conversion of 3-MPA into its dimer, dTdPA. The rate of conversion was affected dramatically by both the presence of QDs and the light environment. 


\section{G) Supplementary Material}

\section{1) Synthesis of lead oleate, $\mathrm{Pb}(\mathrm{Oa})_{2}$}

Lead(II) oleate was prepared via two separate methods. Both methods yielded qualitatively similar results via ${ }^{1} \mathrm{H}-\mathrm{NMR}$ analysis.

\section{Method 1}

Lead(II) oxide and oleic acid in a 1:2 mol:mol ratio were refluxed under nitrogen until a non-scattering slightly yellow oil was obtained. The oil was put under vacuum and dried overnight at $120^{\circ} \mathrm{C}$. The formed oil was highly viscous and flowed only under applied heat from a heat gun.

\section{Method 2}

Lead(II) nitrate and sodium oleate (1:2 mol:mol) were each dissolved in separate vials via sonication in a 1:1 v:v mixture of water and ethanol. The solution containing lead was added dropwise to the sodium oleate solution under vigorous stirring; the addition immediately caused flocculation of a white precipitate. The sample was allowed to stir for $\sim 5$ minutes and then sonicated for $\sim 5$ minutes to disperse the flocculate. The flocculate was allowed to settle and the supernatant was removed via pipette. The leftover solid was dispersed in ethanol via sonication before being flocculated once again by addition of water; after flocculation the suspension was sonicated for 5 minutes to disperse the flocculate as much as possible. The solution was centrifuged (1500 rpm, 5 minutes) to form a pellet with the supernatant water decanted. The pellet was again dissolved in ethanol and the flocculation with water was repeated to wash the product before being centrifuged again. After a 2nd wash cycle, the resulting solid was a loose 
paste with water present. The paste was dispersed in toluene, becoming clear, and centrifuged (5000 rpm, 5 minutes) to separate the water:toluene phases. The toluene phase was removed and lyophilized to yield a white powder.

\section{2) Supplementary Figures}

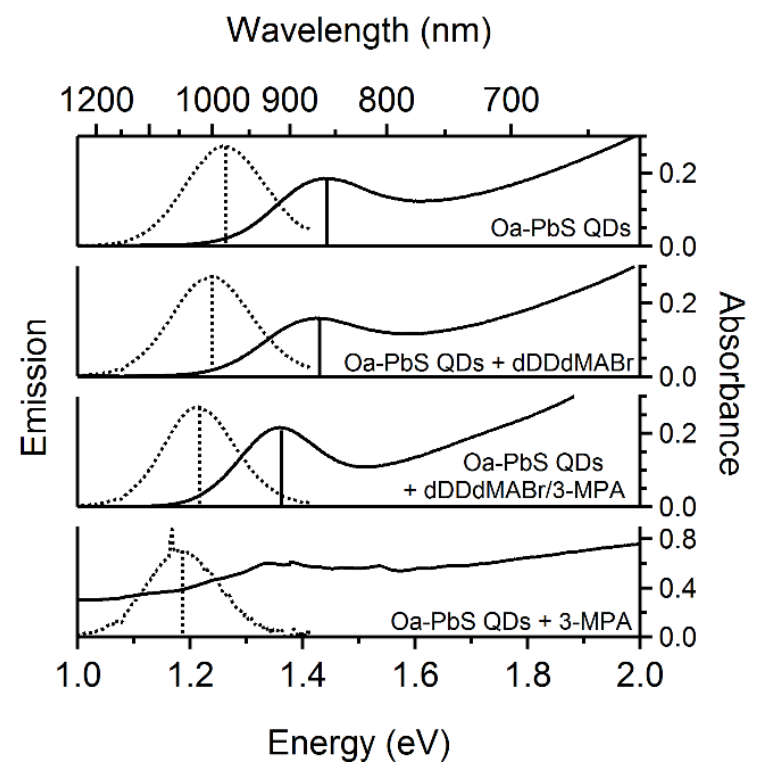

Figure S IV-1. Absorption (solid) and emission (dotted) spectra of Oa-PbS QDs demonstrating excitonic preservation upon addition of a stabilizing additive. Spectra displayed include: Oa-PbS QDs, Oa-PbS QDs re-suspended with dDDdMABr in $\mathrm{CHCl}_{3}$ in a 20:1 ratio of [dDDdMABr]:[Oa- $]$, Oa-PbS QDs re-suspended with 3-MPA and dDDdMABr in $\mathrm{CHCl}_{3}$ in a 20:20:1 ratio of [3-MPA]:[dDDdMABr]:[Oa ${ }^{-}$, and $\mathrm{Oa}-\mathrm{PbS}$ QDs re-suspended with 3-MPA in $\mathrm{CHCl}_{3}$ in a 20:1 ratio of [3-MPA]:[Oa $\left.{ }^{-}\right]$. Vertical lines represent the fit position of the emission peak (dashed) and first excitonic absorption (solid) for each spectrum. The absorbance spectra of Oa-PbS QDs with 3-MPA was not fit as it presented a broad peak from an aggregated suspension. A large spike at $\sim 1.17 \mathrm{eV}$ in the emission spectrum for the suspension with 3-MPA alone is due to scattered light from the fundamental harmonic of the $532 \mathrm{~nm}$ laser used for excitation of the samples. 

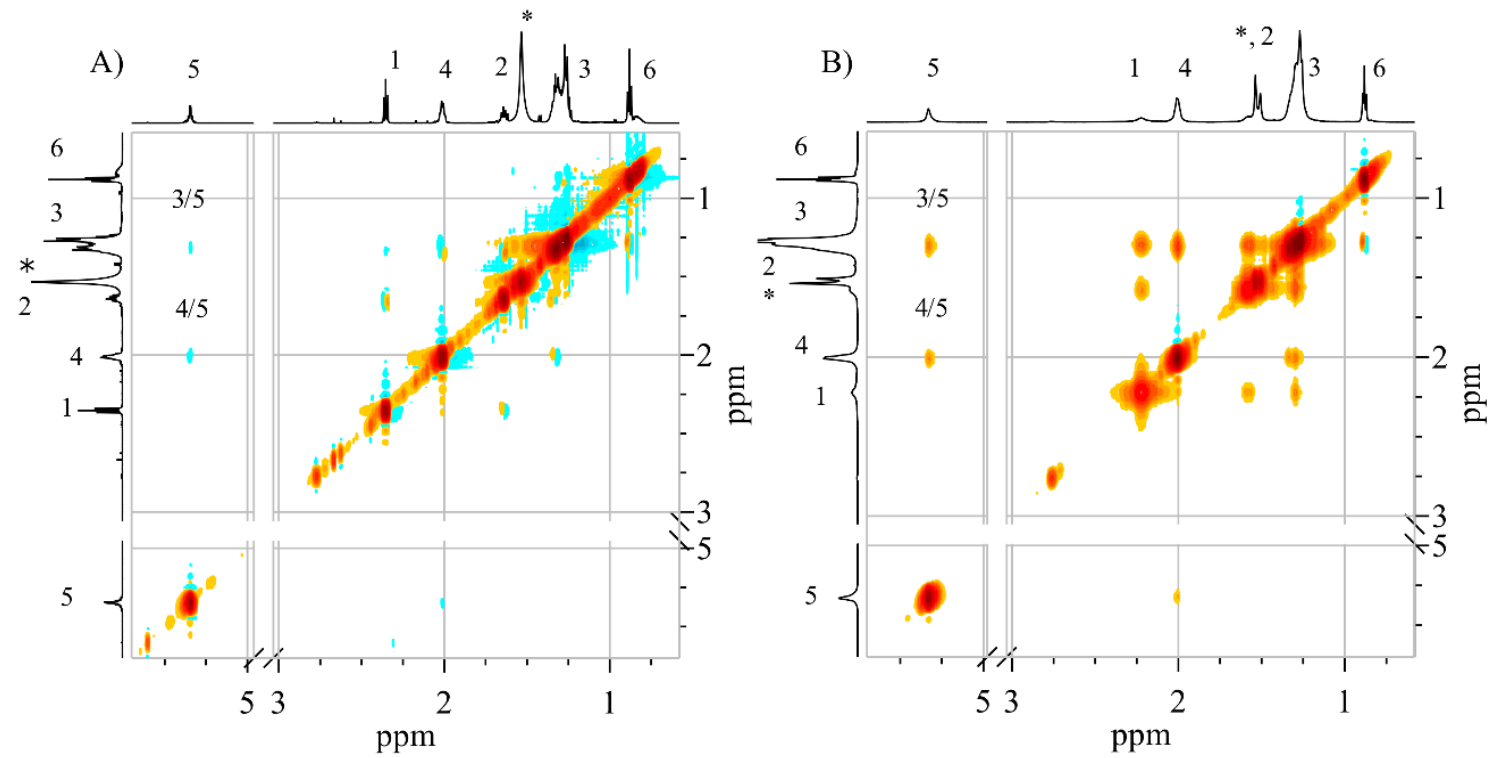

Figure S IV-2. NOESY spectra of oleyl species with and without QDs present. A) a solution of OaH in $\mathrm{CDCl}_{3}$ and $\mathrm{B}$ ) a colloidal suspension of $\mathrm{Oa}-\mathrm{PbS} \mathrm{QDs}$ in $\mathrm{CDCl}_{3}$. Strong negative (same sign as main diagonal) cross peaks indicate species with a critical rotational correlation time similar to that of a QD sized particle whereas weak positive (opposite sign as main diagonal) or antiphase (both negative and positive) cross peaks indicate free molecular species.

Figure S IV-2 demonstrates the differences observed between free species and species bound to QD surfaces as observed by 2-dimensional NOESY. Free molecular species such as $\mathrm{OaH}$ seen in Figure S IV-2A show weak positive or antiphase cross peaks (e.g., 3/5, 4/5 cross peaks). Figure S IV-2B shows Oa-PbS QDs under the same conditions and concentration of oleyl species (either $\mathrm{OaH}$ or $\mathrm{Oa}^{-}$). Strong negative cross peaks are associated with bound species. 


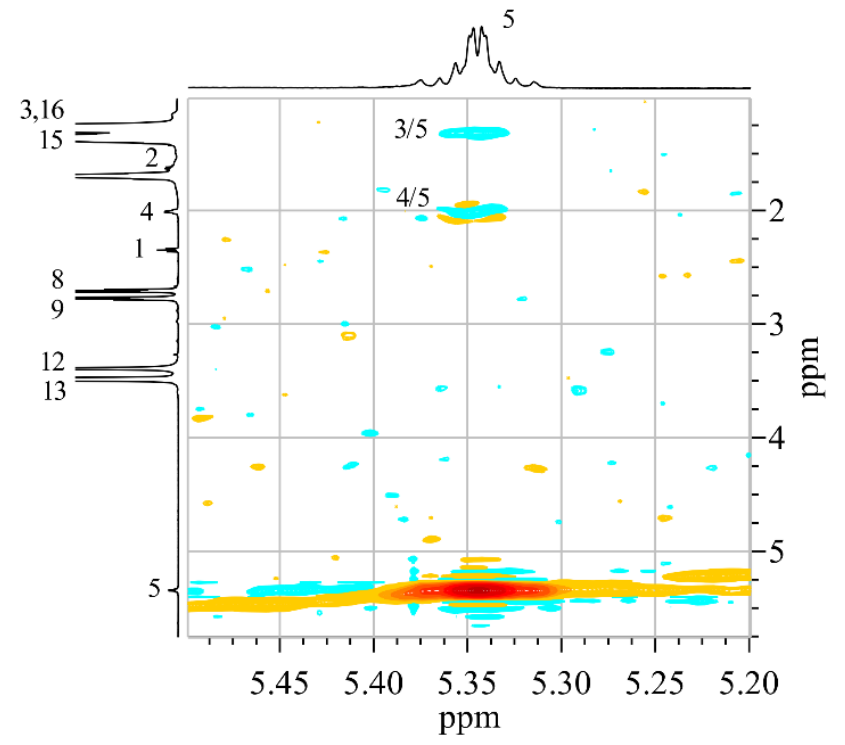

Figure S IV-3. NOESY spectrum of an exchanged QD colloid with a 20:20:1 mixture of [3-

MPA]:[dDDdMABr]:[Oa $\left.{ }^{-}\right]$. This expanded figure focuses on the near noise level cross-peaks from the 
alkene moiety of released oleyl species originally present when bound to Oa-PbS QDs.

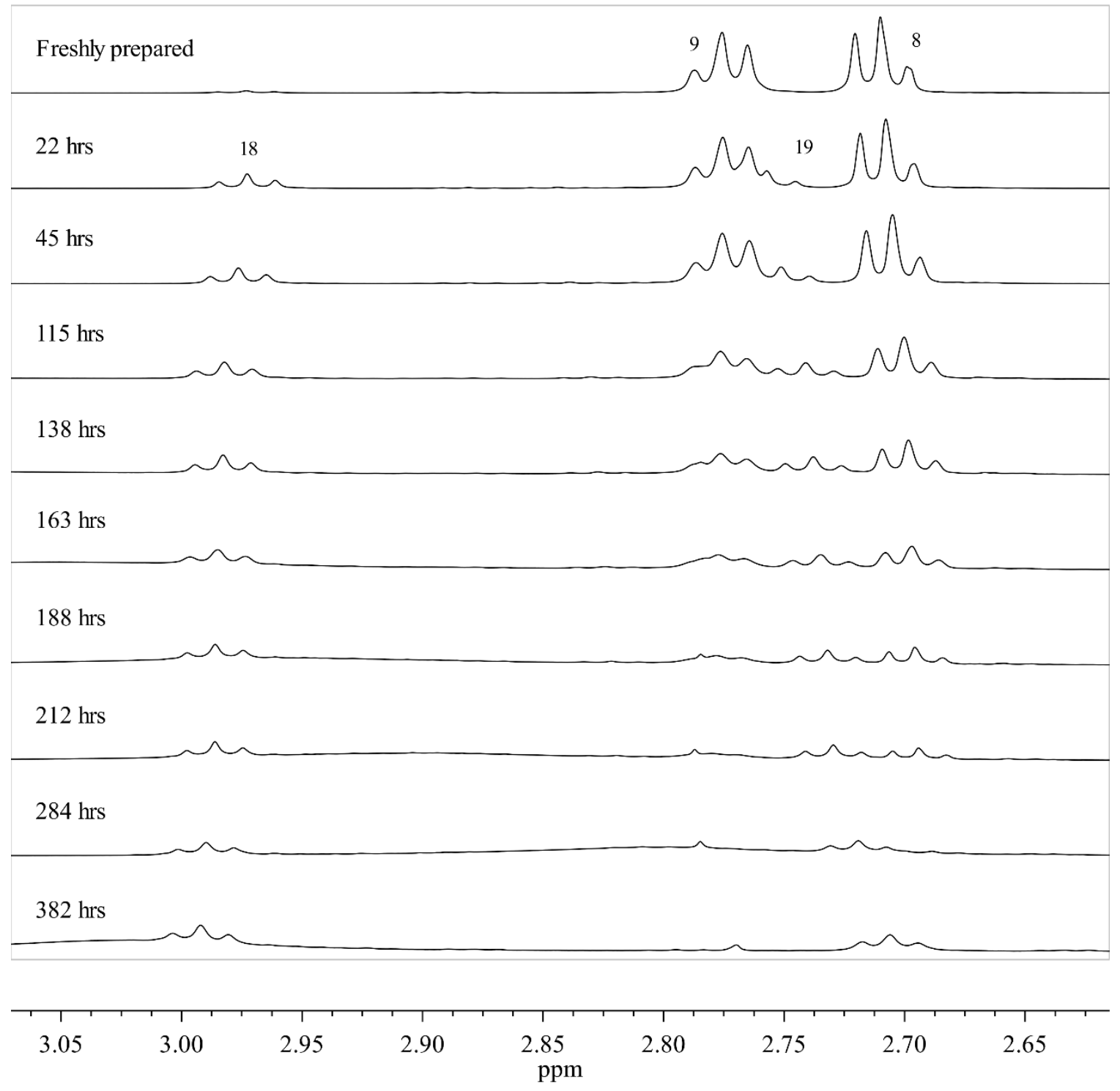

Figure S IV-4. ${ }^{1} \mathrm{H}-\mathrm{NMR}$ spectra of Oa-PbS QDs with 3-MPA/dDDdMABr added in a 20:20:1 ratio of [3-

MPA]:[dDDdMABr]:[Oa $\left.{ }^{-}\right]$. Spectra were acquired on freshly prepared suspensions and then allowed to age in ambient room lighting for the displayed time. 


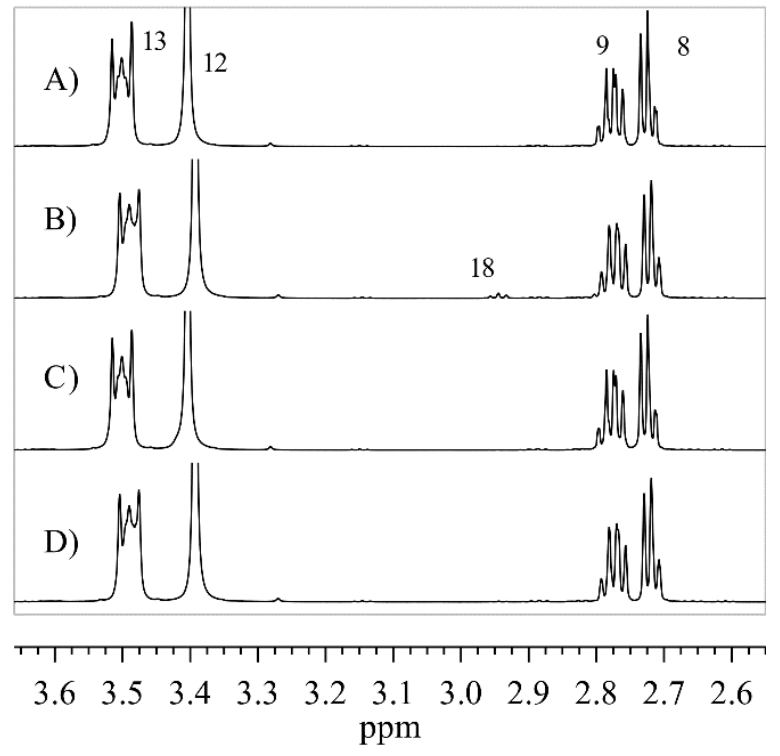

Figure S IV-5. ${ }^{1} \mathrm{H}$ NMR spectra of 3-MPA/dDDdMABr solutions in a 1:1 ratio in $\mathrm{CDCl}_{3}$. Samples were kept in different light environments and allowed to age for 16 days. A) Freshly prepared sample stored in ambient light, B) stored in ambient light for 16 days, C) freshly prepared stored in dark conditions, D) stored in dark conditions for 16 days. 


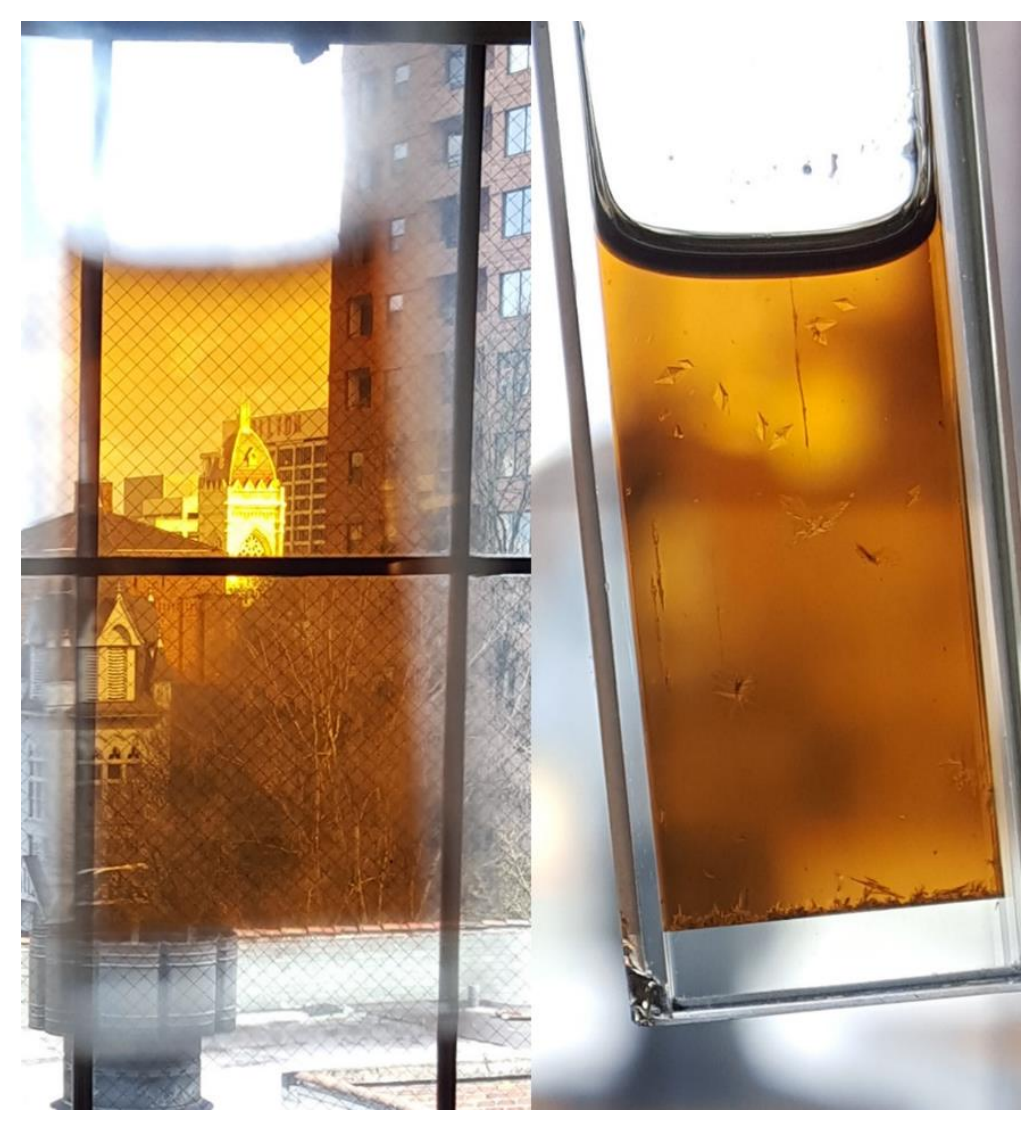

Figure S IV-6. Photograph of Oa-PbS QDs with 3-MPA/dDDdMABr added in a 20:20:1 ratio of [3MPA]:[dDDdMABr]:[Oa- $]$ after $\sim 48$ hours of aging. The suspension remains optically transparent (left), while faceted crystals of dTdPA are observed floating in solution if the focal plane is aimed inside the cuvette (right). 

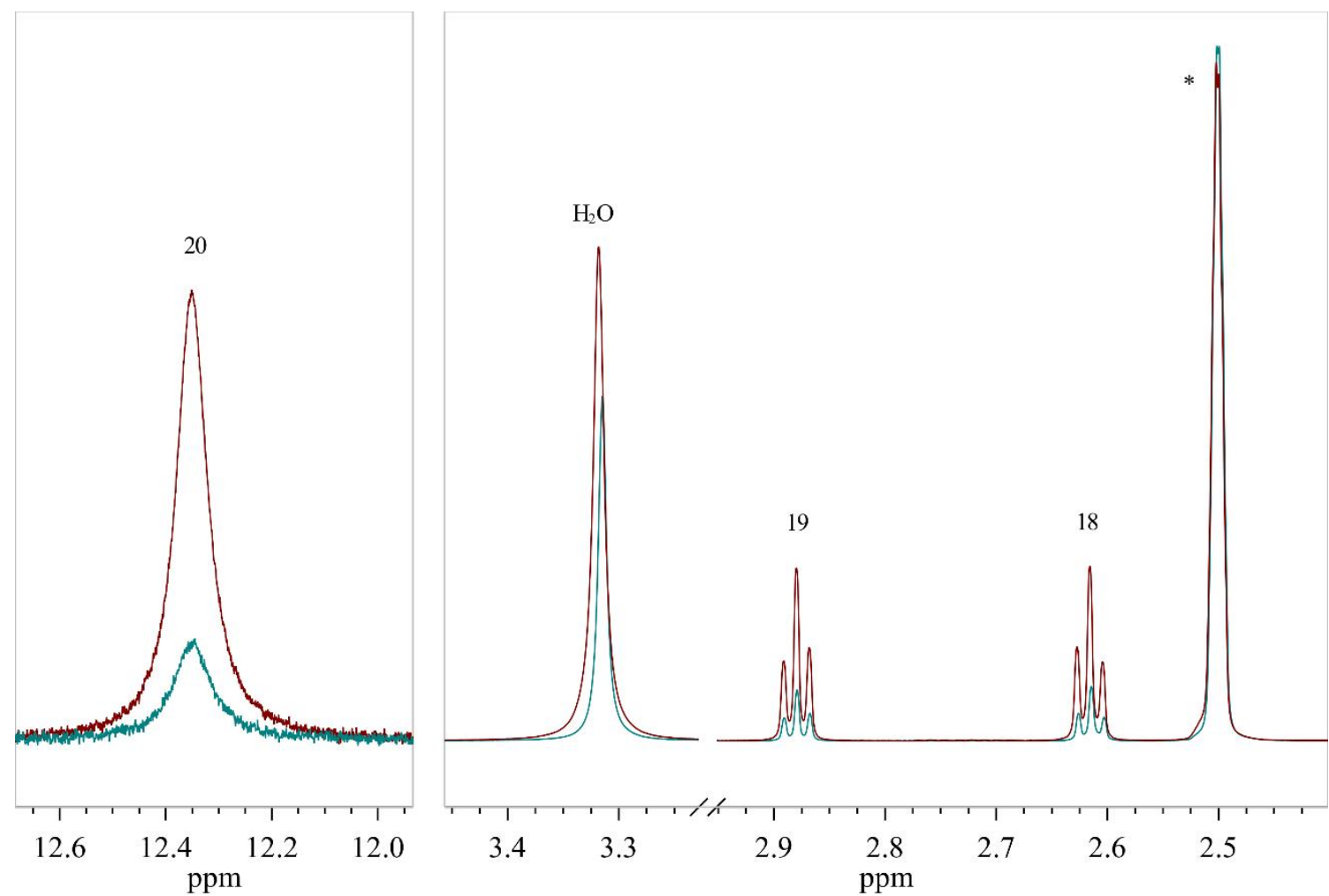

Figure S IV-7. ${ }^{1} \mathrm{H}$ NMR spectra in $\mathrm{D}_{6}$-DMSO of (blue) precipitated solid from aging experiments in $\mathrm{CDCl}_{3}$ and (red) a similar solution spiked with a small grain of commercially obtained dTdPA. Residually protonated DMSO is present at $2.5 \mathrm{ppm}$ and marked with “*”.

Figure S IV-7 shows ${ }^{1} \mathrm{H}-\mathrm{NMR}$ spectra of a recovered crystalline precipitate from aging experiments in $\mathrm{CDCl}_{3}$. The crystal was washed three times with $\mathrm{CDCl}_{3}(\mathrm{dTdPA}$ has low solubility in $\mathrm{CHCl}_{3} / \mathrm{CDCl}_{3}$ ) to remove any free contaminants before being suspended in a $2 \mathrm{~mL}$ solution of DMSO. The solution was split into two samples and a small grain of dTdPA was added as an internal spike to one of the aliquots. The intensity of each peak assigned to dTdPA increased upon addition of the spike. The peak assigned to $\mathrm{H}_{2} \mathrm{O}$ increased as well, which is likely due to water brought in with the spike as the added dTdPA was not extensively dried and is mildly hygroscopic. As a comparison, the 
intensity of the DMSO peak did not change qualitatively or quantitatively in any significant manner.

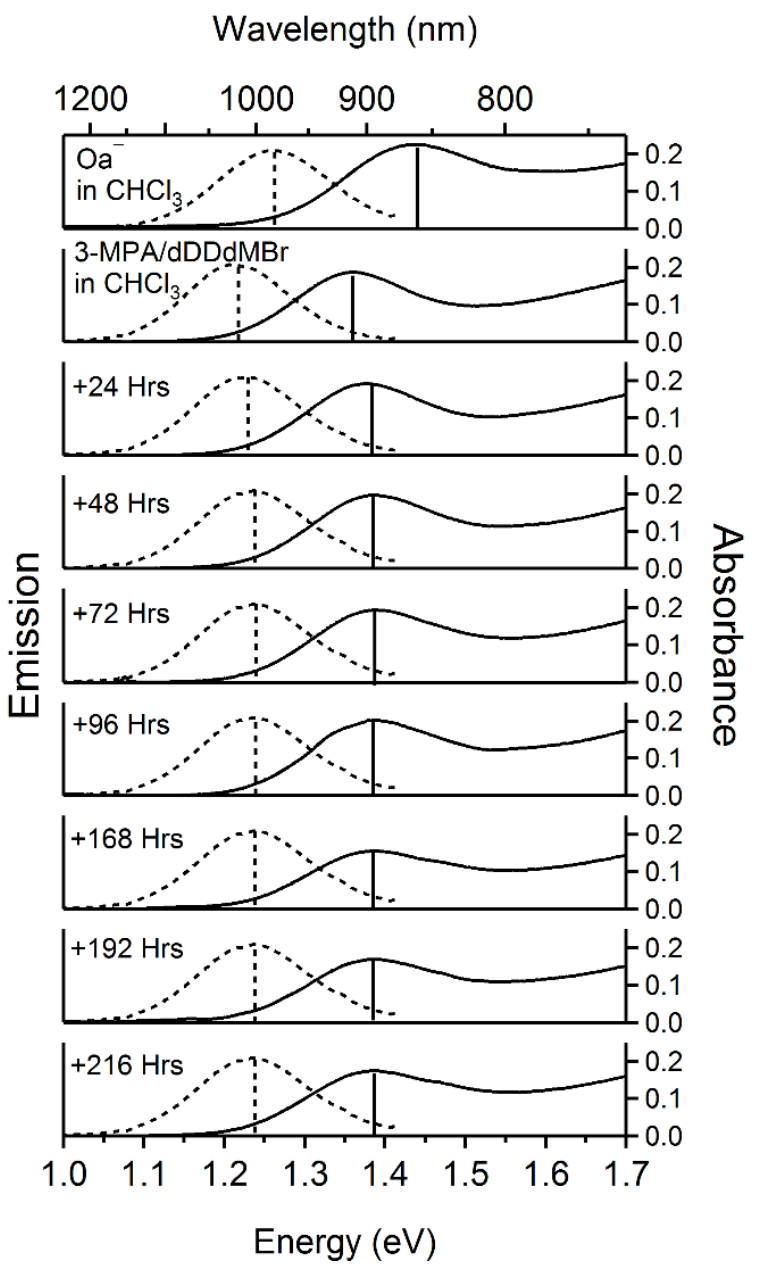

Figure S IV-8. Absorption (solid) and emission (dashed) spectra of Oa-PbS QDs with 3-MPA/dDDdMABr added in a 20:20:1 ratio of [3-MPA]:[dDDdMABr]:[Oa $\left.{ }^{-}\right]$. The colloid was allowed to age for $\sim 9$ days with spectra taken in intervals to follow changes in optical properties. Vertical lines represent the fit position of the emission peak (dashed) and first excitonic absorption (solid) for each spectrum. 


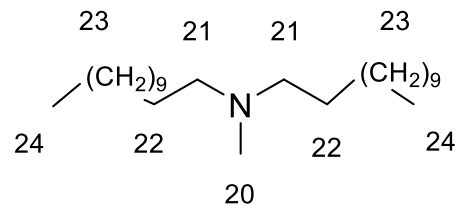

didodecylmethylamine

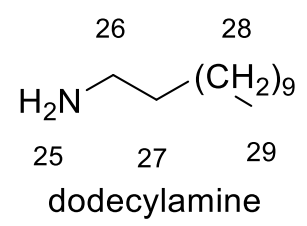

Chart IV-4. Didodecylmethylamine (dDDMA) and dodecylamine (DDA). Each molecule's distinguishable ${ }^{1} \mathrm{H}$ environments are labeled.

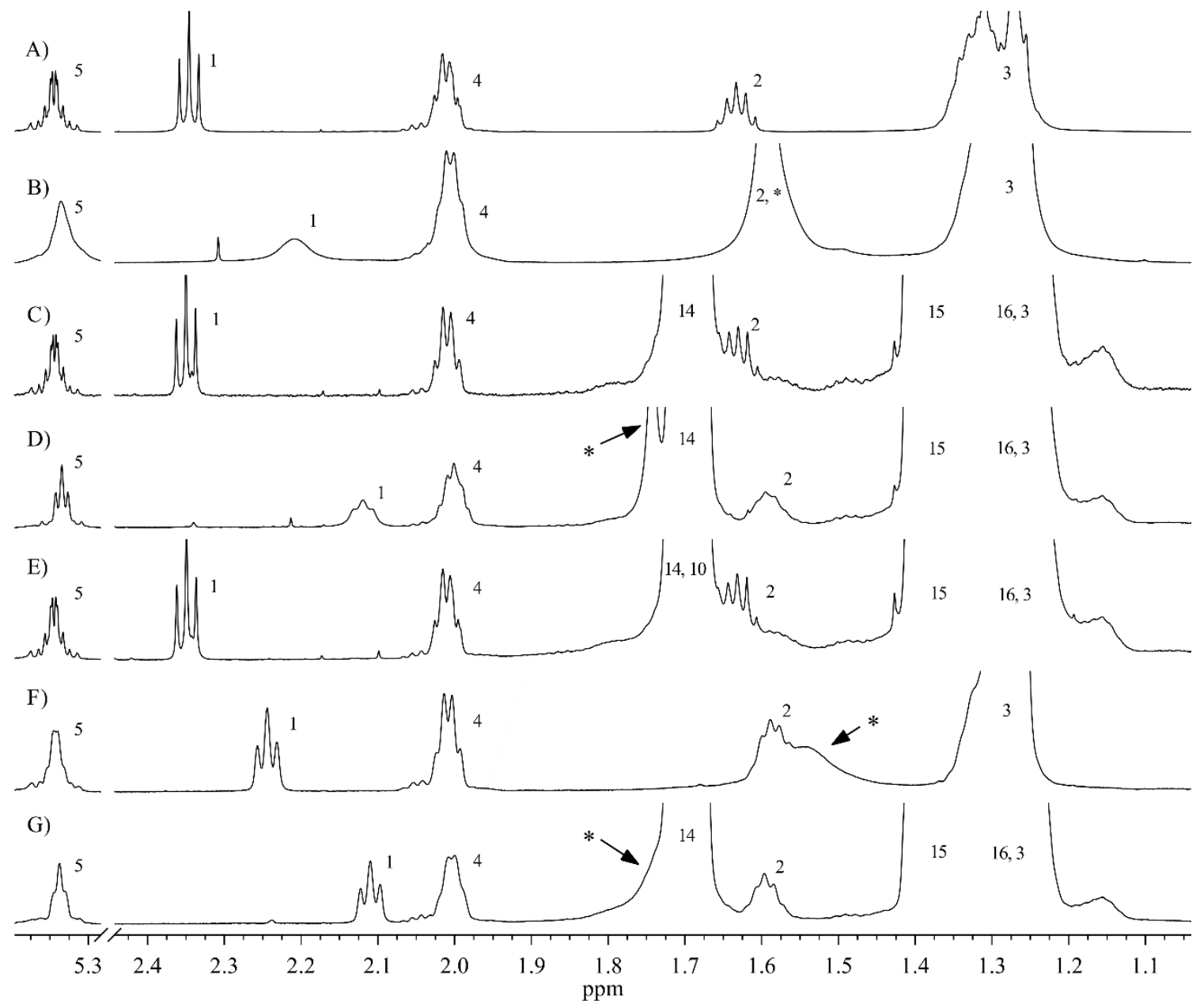

Figure S IV-9. Expanded ${ }^{1} \mathrm{H}$ NMR spectra of oleyl resonances under varying exchange conditions. A) OaH, B) Oa-PbS QDs, C) OaH with dDDdMABr in a 20:1 ratio of [dDDdMABr]:[OaH], D) OA-PbS QDs with dDDdMABr added in a 20:1 ratio of [dDDdMABr]:[OA], E) Oa-PbS QDs with 3-MPA/dDDdMABr added 
in a 20:20:1 ratio of [3-MPA]:[dDDdMABr]:[Oa- $], F) \mathrm{Pb}(\mathrm{Oa})_{2}$, and $\left.\mathrm{G}\right) \mathrm{Pb}(\mathrm{Oa})_{2}$ with dDDdMABr added in a 20:1 ratio of [dDDdMABr]:[Oa- $]$.

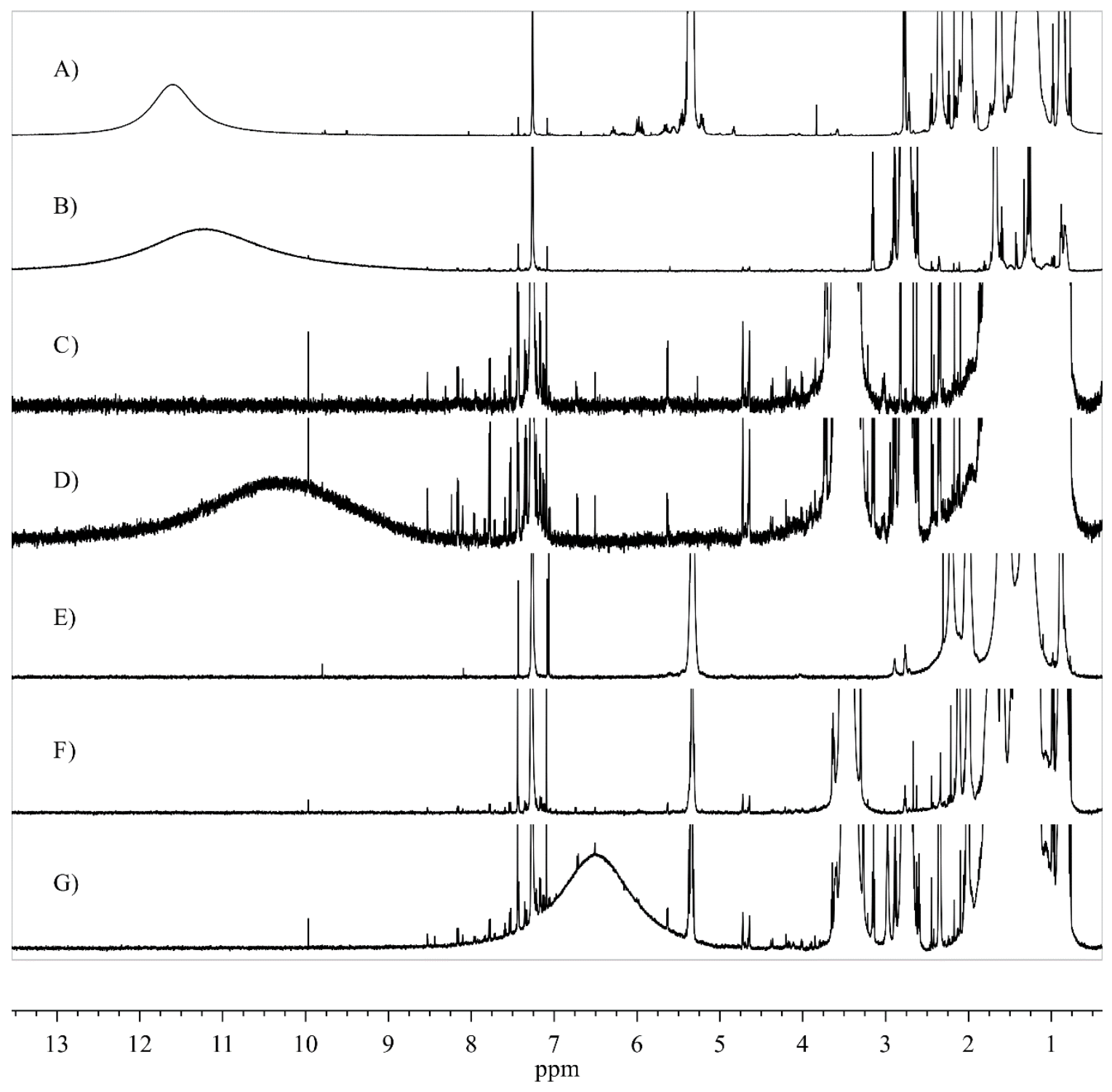

Figure S IV-10. ${ }^{1} \mathrm{H}-\mathrm{NMR}$ in $\mathrm{CDCl}_{3}$ of expanded oleyl species focusing on carboxylic acid resonances. A) OaH, B) 3-MPA, C) dDDdMABr D) a 1:1 mixture of 3-MPA/dDDdMABr, E) Oa-PbS QDs, F) QDs with dDDdMABr added in a 20:1 ratio of [dDDdMABr]:[OA], and G) Oa-PbS QDs with 3-MPA/dDDdMABr added in a 20:20:1 ratio of [3-MPA]:[dDDdMABr]:[Oa $\left.{ }^{-}\right]$. The concentration of oleyl species is the same in all spectra. 
Figure S IV-10 is vertically expanded to highlight $\mathrm{COOH}$ and exchangeable proton peaks present in some of the samples. Both 3-MPA and OaH have acidic carboxylic acid groups that can deprotonate. Oa-PbS QDs in Figure S IV-10E do not have any resolvable acidic protons, consistent with previous reports of carboxylate binding. ${ }^{107}$ Figure S IV-10F does not show any resolvable acidic protons, indicating that dDDdMABr does not release free $\mathrm{OaH}$ in a significant enough magnitude to observe free $\mathrm{OaH}$. 


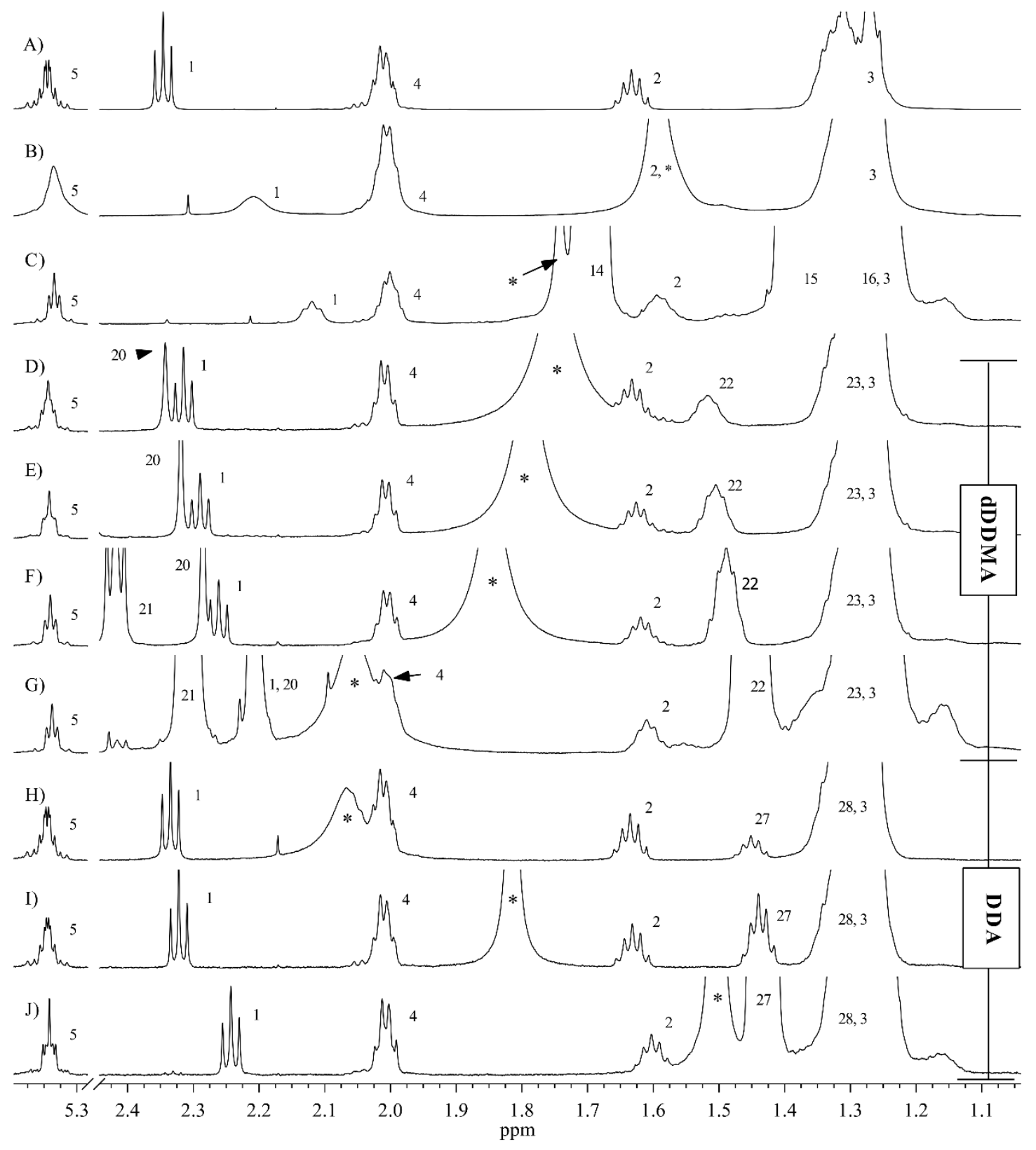

Figure S IV-11. Expanded ${ }^{1} \mathrm{H}$ NMR spectra of oleyl resonances upon addition of amines. A) OaH, B) OaPbS QDs, C) OA-PbS QDs with dDDdMABr added in a 20:1 ratio of [dDDdMABr]:[OA],

Didodecylmethylamine (dDDMA) with $\mathrm{OaH}$ in D) a 1:2, E) 1:1, F) 2:1, G) 20:1 ratio of [dDDMA]:[OaH], and Dodecylamine (DDA) with $\mathrm{OaH}$ in $\mathrm{H}$ ) a 1:2, I) 2:1, J) and 20:1 ratio of [DoDA]:[OaH] respectively. The concentration of oleyl species is the same in all spectra. A mobile peak attributed to either water or water/amine exchange in samples with amines is present in some samples and marked with “*”. 

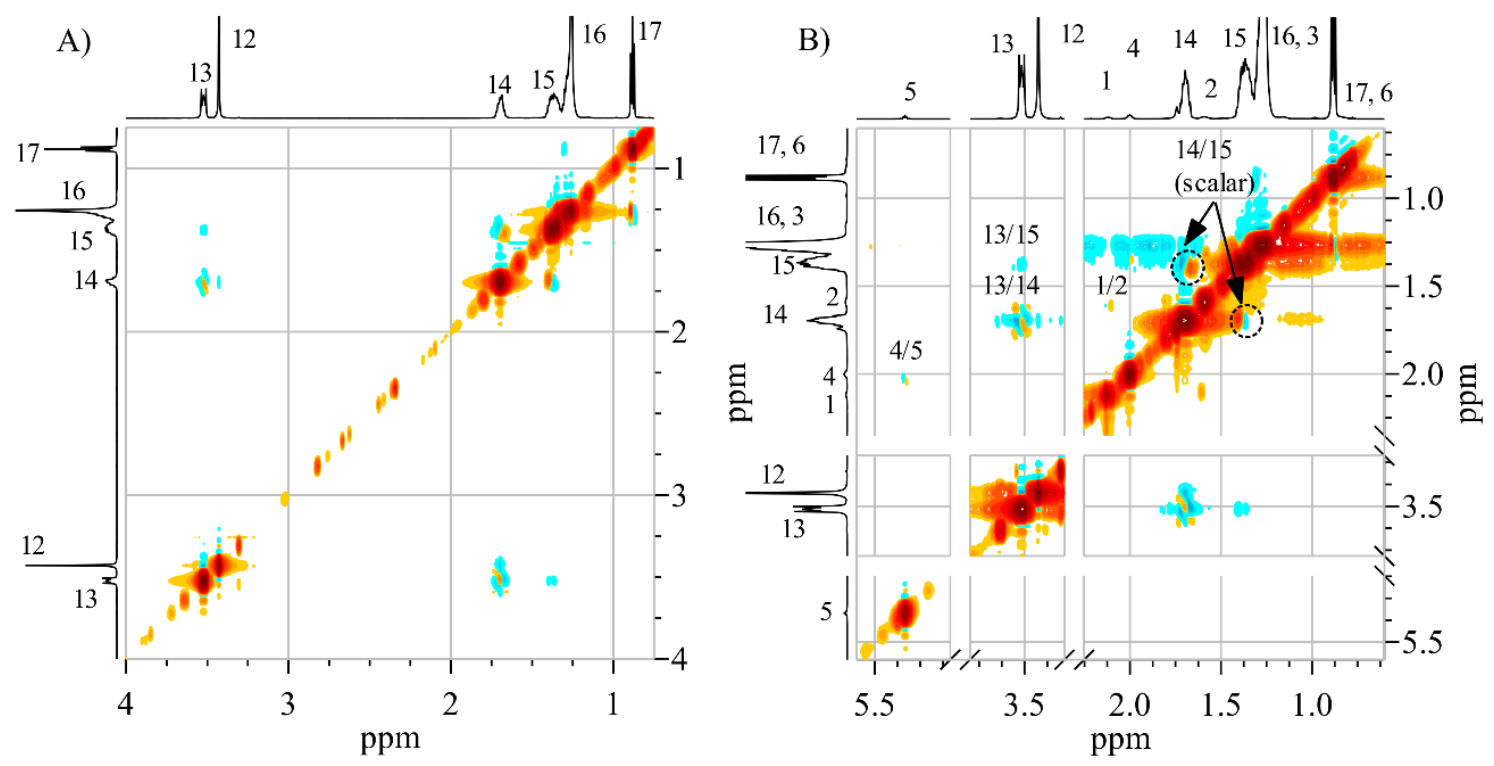

Figure S IV-12. NOESY spectrum of A) a solution of dDDdMABr in $\mathrm{CDCl}_{3}$ and $\mathrm{B}$ ) an $\mathrm{OA}-\mathrm{PbS} \mathrm{QD}$ colloid in $\mathrm{CDCl}_{3}$ with dDDdMABr added to solution in a 20:1 [dDDdMABr]:[Oa $\left.{ }^{-}\right]$ratio. The concentration of dDDdMABr and $\mathrm{Oa}^{-}$is the same in both spectra.

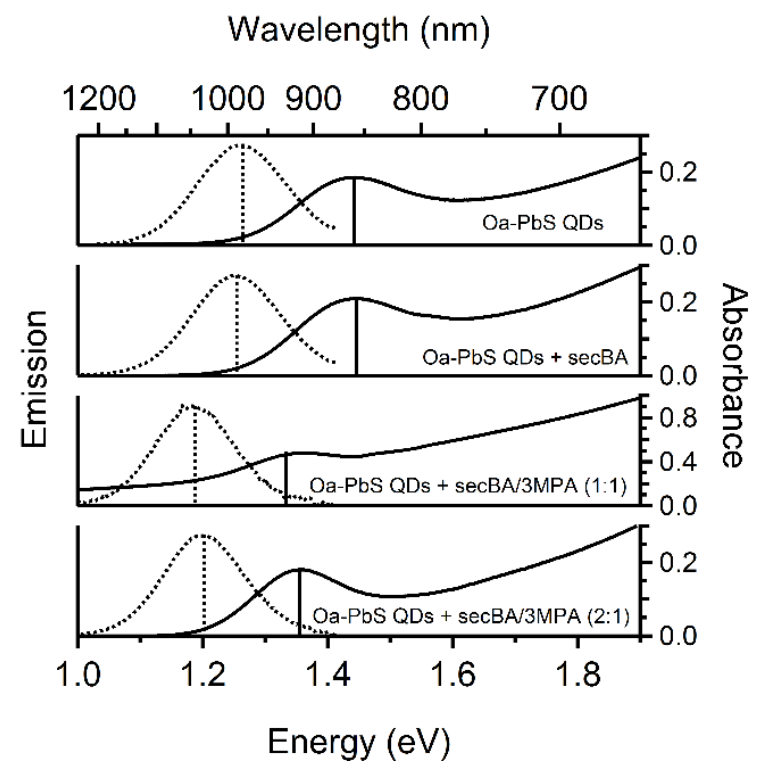


Figure S IV-13. Absorption (solid) and emission (dashed) spectra of Oa-PbS QDs upon exposure to 3-MPA and sec-butylamine in $\mathrm{CHCl}_{3}$. Spectra displayed are: Oa-PbS QDs, Oa-PbS QDs re-suspended with secBA added in a 20:1 ratio of [secBA]:[Oa $\left.{ }^{-}\right]$, Oa-PbS QDs re-suspended with secBA/3-MPA added in a 20:20:1 ratio of [secBA]:[3-MPA]:[Oa ${ }^{-}$, and Oa-PbS QDs re-suspended with secBA/3-MPA added in a 40:20:1 ratio of $[\operatorname{secBA}]:[3-\mathrm{MPA}]:\left[\mathrm{Oa}^{-}\right]$. Vertical lines represent the fit position of the emission peak (dashed) and first excitonic absorption (solid) for each spectrum. 


\section{V) CONCLUSIONS AND FUTURE DIRECTIONS}

\section{A) Conclusions}

Colloidal suspensions of 3-MPA capped PbS QDs were investigated in a variety of organic solvents with the aim of formulating a directly depositable 3-MPA capped PbS QD “ink”. Initial suspensions in DMSO proved to be difficult to use for solution processing. Colloids of 3-MPA capped PbS QDs were subsequently obtained in volatile organic solvents that are more amenable to common solution processing techniques for the formation of QD solids arrays. In both cases, the PbS QDs were highly catalytic towards the photo-driven conversion of monomer 3-MPA to dimerized dTdPA.

Ultimately, the addition of a stabilizing amine/ammonium allowed preparation of 3-MPA capped PbS QD colloids in a variety of solvents that were able to form conformal coatings in single step deposition processes (e.g., spin coating, drop-cast evaporative deposition); initial attempts to use this "ink" in preparing solar devices have yet to be explored extensively. While successful inks were obtained, the lack of significant crosslinking in directly deposited films may ultimately hinder highly coupled film formation.

\section{B) Future Directions}

\section{1) Thermal Processing}

The addition of dDDdMABr as a stabilizer re-introduces a problem for optoelectronic applications. Long alkane surfactants, typically used to confer stability in low-polarity solvents by direct ligation to a QD surface, are electronically insulating and mitigate facile charge transport in QD films. dDDdMABr has previously been thermally removed 
from QD films without disrupting film order. ${ }^{137}$ Thermal processing of directly deposited films remains a viable option for removing stabilizing additives while maintaining film order and offers a simple approach to device fabrication.

\section{2) Alternate stabilizers}

Sec-butylamine and dDDdMABr were chosen as exploratory options for colloidal stabilization of 3-MPA capped PbS QDs in organic solvents but are merely part of a larger library of possible stabilizers. A wide spectrum of amine based additives present themselves readily. Long chain primary amines have been shown to increase binding strength of carboxylic acid based QD ligands. ${ }^{24}$ Additionally, volatile $\mathrm{NH}_{4}{ }^{+}$ions have also been used to charge balance anionic ligand shells. ${ }^{67,68}$ Given the variety of possible amine/ammonium based additives, a massive library of application focused chemical combinations remains.

\section{C) Final Thoughts}

The limitations of an "ink" based approach to QD solid formation for optoelectronic applications do not escape the author: The formulated inks, while colloidally stable, demonstrate photocatalytic consumption of the ligand of interest for PV applications; the lack of significant cross-linking in QD films precludes the opportunity for bifunctional ligand coupling of QDs; the addition of an organic stabilizer allows for direct deposition but may interfere with optoelectronic performance of as deposited devices. These problems, and others, are viewed as smaller obstacles for device engineering in the face of the newly developed ability to provide solution processability to a previously unobtained colloidal QD-ligand that has largely driven a new field of PV devices in the 
last decade. That is, colloidal control is viewed as a pre-requitiste condition for any successful "bottom up" assembly approach. 


\section{References}

(1) IEA. Key World Energy Statistics 2015; Key World Energy Statistics; OECD Publishing, 2015.

(2) U.S. Energy Information Administration. Annual Energy Outlook 2015. Off. Integr. Int. Energy Anal. 2015, 1, 1-244.

(3) IEA. World Energy Outlook 2015; World Energy Outlook; OECD Publishing, 2015.

(4) Lewis, N. S.; Crabtree, G.; Nozik, A. J.; Wasielewski, M. R.; Alivisatos, A. P. Basic Research Needs for Solar Energy Utilization. Basic Energy Sci. Work. Sol. Energy Util. 2005, 276.

(5) Lewis, N. S.; Nocera, D. G. Powering the Planet: Chemical Challenges in Solar Energy Utilization. Proc. Natl. Acad. Sci. 2006, 103 (43), 15729-15735.

(6) Green, M. A.; Emery, K.; Hishikawa, Y.; Warta, W.; Dunlop, E. D. Solar Cell Efficiency Tables (Version 47). Prog. Photovoltaics Res. Appl. 2016, 24 (1), 3-11.

(7) Chapin, D. M.; Fuller, C. S.; Pearson, G. L. A New Silicon P-N Junction Photocell for Converting Solar Radiation into Electrical Power. J. Appl. Phys. 1954, 25 (5), 676.

(8) Krishnan Rajeshwar. Fundamentals of Semiconductor Electrochemistry and Photoelectrochemistry. In Encyclopedia of Electrochemistry; Wiley-VCH Verlag GmbH \& Co. KGaA: Weinheim, Germany, 2007.

(9) Tan, M. X.; Laibinis, P. E.; Nguyen, S. T.; Kesselman, J. M.; Stanton, C. E.; Lewis, N. S. Principles and Applications of Semiconductor Photoelectrochemistry. 
In Progress in ...; 1994; Vol. 41, pp 21-144.

(10) Kitamura, M.; Muramatsu, S.; Harrison, W. A. Elastic Properties of Semiconductors Studied by Extended Hückel Theory. Phys. Rev. B 1992, 46 (3), $1351-1357$.

(11) Kienle, D.; Cerda, J. I.; Ghosh, A. W. Extended Hückel Theory for Band Structure, Chemistry, and Transport. I. Carbon Nanotubes. J. Appl. Phys. 2006, 100 (4), 43714.

(12) Bard, A. J.; Faulkner, L. R. Electrochemical Methods: Fundamentals and Applications, Second Edi.; John Wiley \& Sons, Inc: Hoboken, New Jeyser, 2001.

(13) Miessler, G. L.; Tarr, D. A. Inorganic Chemistry, Third Edit.; Pearson Education, Inc: Upper Saddle River, New Jersey, 2004.

(14) Neamen, D. A. Semiconductor Physics and Devices: Basic Principles, Fourth Edi.; New York, NY, 2012.

(15) Best Research Cell Efficiencies http://www.nrel.gov/ncpv/images/efficiency_chart.jpg.

(16) de Mello Donegá, C. Nanoparticles: Workhorses of Nanoscience; 2014.

(17) Brus, L. E. A Simple Model for the Ionization Potential, Electron Affinity, and Aqueous Redox Potentials of Small Semiconductor Crystallites. J. Chem. Phys. 1983, $79(11), 5566$.

(18) Alivisatos, A. P. Perspectives on the Physical Chemistry of Semiconductor Nanocrystals. J. Phys. Chem. 1996, 100 (95), 13226-13239.

(19) Wise, F. W. Lead Salt Quantum Dots: The Limit of Strong Quantum Confinement. 
Acc. Chem. Res. 2000, 33 (11), 773-780.

(20) Valdez, C. N.; Schimpf, A. M.; Gamelin, D. R.; Mayer, J. M. Low Capping Group Surface Density on Zinc Oxide Nanocrystals. ACS Nano 2014, No. Xx.

(21) Zherebetskyy, D.; Scheele, M.; Zhang, Y.; Bronstein, N.; Thompson, C.; Britt, D.; Salmeron, M.; Alivisatos, A. P.; Wang, L.-W. Hydroxylation of the Surface of PbS Nanocrystals Passivated with Oleic Acid. Science (80-. ). 2014, 344 (6190), 13801384.

(22) Anderson, N. C.; Hendricks, M. P.; Choi, J. J.; Owen, J. S. Ligand Exchange and the Stoichiometry of Metal Chalcogenide Nanocrystals: Spectroscopic Observation of Facile Metal-Carboxylate Displacement and Binding. J. Am. Chem. Soc. 2013, 135 (49), 18536-18548.

(23) Khan, A. H.; Thupakula, U.; Dalui, A.; Maji, S.; Debangshi, A.; Acharya, S. Evolution of Long Range Bandgap Tunable Lead Sulfide Nanocrystals with Photovoltaic Properties. J. Phys. Chem. C 2013, 117 (15), 7934-7939.

(24) Grisorio, R.; Debellis, D.; Suranna, G. P.; Gigli, G.; Giansante, C. The Dynamic Organic/Inorganic Interface of Colloidal PbS Quantum Dots. Angew. Chemie Int. Ed. 2016, 55 (23), 6628-6633.

(25) Wang, Y.; Tang, A.; Li, K.; Yang, C.; Wang, M.; Ye, H.; Hou, Y.; Teng, F. ShapeControlled Synthesis of PbS Nanocrystals via a Simple One-Step Process. Langmuir 2012, 28 (47), 16436-16443.

(26) Bertolotti, F.; Dirin, D. N.; Ibáñez, M.; Krumeich, F.; Cervellino, A.; Frison, R.; Voznyy, O.; Sargent, E. H.; Kovalenko, M. V.; Guagliardi, A.; Masciocchi, N. 
Crystal Symmetry Breaking and Vacancies in Colloidal Lead Chalcogenide Quantum Dots. Nat. Mater. 2016, 15 (9), 987-994.

(27) Moreels, I.; Lambert, K.; Smeets, D.; De Muynck, D.; Nollet, T.; Martins, J. C.; Vanhaecke, F.; Vantomme, A.; Delerue, C.; Allan, G.; Hens, Z. Size-Dependent Optical Properties of Colloidal PbS Quantum Dots. ACS Nano 2009, 3 (10), $3023-$ 3030.

(28) Leatherdale, C. A.; Bawendi, M. G. Observation of Solvatochromism in CdSe Colloidal Quantum Dots. Phys. Rev. B 2001, 63 (16), 165315.

(29) Wuister, S. F.; de Mello Donega, C.; Meijerink, A. Local-Field Effects on the Spontaneous Emission Rate of CdTe and CdSe Quantum Dots in Dielectric Media. J. Chem. Phys. 2004, 121 (9), 4310-4315.

(30) Litvin, A. P.; Parfenov, P. S.; Ushakova, E. V; Simões Gamboa, A. L.; Fedorov, A. V; Baranov, A. V. Size and Temperature Dependencies of the Low-Energy Electronic Structure of PbS Quantum Dots. J. Phys. Chem. C 2014, 118 (35), 20721-20726.

(31) Gao, J.; Johnson, J. C. Charge Trapping in Bright and Dark States of Coupled PbS Quantum Dot Films. ACS Nano 2012, 6 (4), 3292-3303.

(32) Wolcott, A.; Doyeux, V.; Nelson, C. A.; Gearba, R.; Lei, K. W.; Yager, K. G.; Dolocan, A. D.; Williams, K.; Nguyen, D.; Zhu, X.-Y. Anomalously Large Polarization Effect Responsible for Excitonic Red Shifts in PbSe Quantum Dot Solids. J. Phys. Chem. Lett. 2011, 2 (7), 795-800.

(33) Amin, V. A.; Aruda, K. O.; Lau, B.; Rasmussen, A. M.; Edme, K.; Weiss, E. A. 
Dependence of the Band Gap of CdSe Quantum Dots on the Surface Coverage and Binding Mode of an Exciton-Delocalizing Ligand, Methylthiophenolate. J. Phys.

Chem. C 2015, 119 (33), 19423-19429.

(34) Frederick, M. T.; Amin, V. a; Swenson, N. K.; Ho, A. Y.; Weiss, E. A. Control of Exciton Confinement in Quantum Dot-Organic Complexes through Energetic Alignment of Interfacial Orbitals. Nano Lett. 2013, 13 (1), 287-292.

(35) Morris-Cohen, A. J.; Vasilenko, V.; Amin, V. A.; Reuter, M. G.; Weiss, E. A. Model for Adsorption of Ligands to Colloidal Quantum Dots with ConcentrationDependent Surface Structure. ACS Nano 2012, 6 (1), 557-565.

(36) Nepomnyashchii, A. B.; Harris, R. D.; Weiss, E. A. Composition and Permeability of Oleate Adlayers of CdS Quantum Dots upon Dilution to PhotoluminescenceRelevant Concentrations. Anal. Chem. 2016, 88 (6), 3310-3316.

Harris, R. D.; Amin, V. A.; Lau, B.; Weiss, E. A. Role of Interligand Coupling in Determining the Interfacial Electronic Structure of Colloidal CdS Quantum Dots. ACS Nano 2016, 10 (1), 1395-1403.

(38) Hines, M. A.; Scholes, G. D. Colloidal PbS Nanocrystals with Size-Tunable NearInfrared Emission: Observation of Post-Synthesis Self-Narrowing of the Particle Size Distribution. Adv. Mater. 2003, 15 (21), 1844-1849.

(39) Cademartiri, L.; Bertolotti, J.; Sapienza, R.; Wiersma, D. S.; Von Freymann, G.; Ozin, G. a. Multigram Scale, Solventless, and Diffusion-Controlled Route to Highly Monodisperse PbS Nanocrystals. J. Phys. Chem. B 2006, 110, 671-673.

(40) Moreels, I.; Justo, Y.; De Geyter, B.; Haustraete, K.; Martins, J. C.; Hens, Z. Size- 
Tunable, Bright, and Stable PbS Quantum Dots: A Surface Chemistry Study. ACS Nano 2011, 5 (3), 2004-2012.

(41) Liu, J.; Yu, H.; Wu, Z.; Wang, W.; Peng, J.; Cao, Y. Size-Tunable near-Infrared PbS Nanoparticles Synthesized from Lead Carboxylate and Sulfur with Oleylamine as Stabilizer. Nanotechnology 2008, 19 (34), 345602.

(42) Weidman, M. C.; Beck, M. E.; Hoffman, R. S.; Prins, F.; Tisdale, W. A. Monodisperse, Air-Stable PbS Nanocrystals via Precursor Stoichiometry Control. ACS Nano 2014, 8 (6), 6363-6371.

(43) Cademartiri, L.; Montanari, E.; Calestani, G.; Migliori, A.; Guagliardi, A.; Ozin, G. a. Size-Dependent Extinction Coefficients of PbS Quantum Dots. J. Am. Chem. Soc. 2006, 128 (31), 10337-10346.

(44) Clark, S. W.; Harbold, J. M.; Wise, F. W. Resonant Energy Transfer in PbS Quantum Dots. J. Phys. Chem. C 2007, 111 (20), 7302-7305.

(45) Pattantyus-Abraham, A. G.; Kramer, I. J.; Barkhouse, A. R.; Wang, X.; Konstantatos, G.; Debnath, R.; Levina, L.; Raabe, I.; Nazeeruddin, M. K.; Grätzel, M.; Sargent, E. H. Depleted-Heterojunction Colloidal Quantum Dot Solar Cells. ACS Nano 2010, 4 (6), 3374-3380.

(46) Robel, I.; Kuno, M.; Kamat, P. V. Size-Dependent Electron Injection from Excited CdSe Quantum Dots into TiO 2 Nanoparticles. J. Am. Chem. Soc. 2007, 129 (14), $4136-4137$.

(47) Hyun, B.-R.; Zhong, Y.-W.; Bartnik, A. C.; Sun, L.; Abruña, H. D.; Wise, F. W.; Goodreau, J. D.; Matthews, J. R.; Leslie, T. M.; Borrelli, N. F. Electron Injection 
from Colloidal PbS Quantum Dots into Titanium Dioxide Nanoparticles. ACS Nano 2008, 2 (11), 2206-2212.

(48) Clifford, J. P.; Johnston, K. W.; Levina, L.; Sargent, E. H. Schottky Barriers to Colloidal Quantum Dot Films. Appl. Phys. Lett. 2007, 91, 5-7.

(49) McDonald, S. a; Konstantatos, G.; Zhang, S.; Cyr, P. W.; Klem, E. J. D.; Levina, L.; Sargent, E. H. Solution-Processed PbS Quantum Dot Infrared Photodetectors and Photovoltaics. Nat. Mater. 2005, 4 (2), 138-142.

(50) Johnston, K. W.; Pattantyus-Abraham, A. G.; Clifford, J. P.; Myrskog, S. H.; MacNeil, D. D.; Levina, L.; Sargent, E. H. Schottky-Quantum Dot Photovoltaics for Efficient Infrared Power Conversion. Appl. Phys. Lett. 2008, 92, 5-7.

(51) Liu, M.; de Arquer, F. P. G.; Li, Y.; Lan, X.; Kim, G.-H.; Voznyy, O.; Jagadamma, L. K.; Abbas, A. S.; Hoogland, S.; Lu, Z.; Kim, J. Y.; Amassian, A.; Sargent, E. H. Double-Sided Junctions Enable High-Performance ColloidalQuantum-Dot Photovoltaics. Adv. Mater. 2016, 28 (21), 4142-4148.

(52) Ip, A. H.; Kiani, A.; Kramer, I. J.; Voznyy, O.; Movahed, H. F.; Levina, L.; Adachi, M. M.; Hoogland, S.; Sargent, E. H. Infrared Colloidal Quantum Dot Photovoltaics via Coupling Enhancement and Agglomeration Suppression. ACS Nano 2015, 9 (9), 8833-8842.

(53) Kim, J. Y.; Adinolfi, V.; Sutherland, B. R.; Voznyy, O.; Kwon, S. J.; Kim, T. W.; Kim, J.; Ihee, H.; Kemp, K.; Adachi, M.; Yuan, M.; Kramer, I.; Zhitomirsky, D.; Hoogland, S.; Sargent, E. H. Single-Step Fabrication of Quantum Funnels via Centrifugal Colloidal Casting of Nanoparticle Films. Nat. Commun. 2015, 6, 7772. 
(54) Carey, G. H.; Levina, L.; Comin, R.; Voznyy, O.; Sargent, E. H. Record Charge Carrier Diffusion Length in Colloidal Quantum Dot Solids via Mutual Dot-To-Dot Surface Passivation. Adv. Mater. 2015, 27 (21), 3325-3330.

Labelle, A. J.; Thon, S. M.; Kim, J. Y.; Lan, X.; Zhitomirsky, D.; Kemp, K. W.;

Sargent, E. H. Conformal Fabrication of Colloidal Quantum Dot Solids for Optically Enhanced Photovoltaics. ACS Nano 2015, 9 (5), 5447-5453.

(56) Kim, G.-H.; Walker, B.; Zhitomirsky, D.; Heo, J.; Ko, S.-J.; Park, J.; Sargent, E. H.; Young Kim, J. Synergistic Photocurrent Addition in Hybrid Quantum Dot: Bulk Heterojunction Solar Cells. Nano Energy 2015, 13, 491-499.

(57) Labelle, A. J.; Thon, S. M.; Masala, S.; Adachi, M. M.; Dong, H.; Farahani, M.; Ip, A. H.; Fratalocchi, A.; Sargent, E. H. Colloidal Quantum Dot Solar Cells Exploiting Hierarchical Structuring. Nano Lett. 2015, 15 (2), 1101-1108.

(58) Kramer, I. J.; Minor, J. C.; Moreno-Bautista, G.; Rollny, L.; Kanjanaboos, P.; Kopilovic, D.; Thon, S. M.; Carey, G. H.; Chou, K. W.; Zhitomirsky, D.; Amassian, A.; Sargent, E. H. Efficient Spray-Coated Colloidal Quantum Dot Solar Cells. Adv. Mater. 2015, 27 (1), 116-121.

(59) Ning, Z.; Dong, H.; Zhang, Q.; Voznyy, O.; Sargent, E. H. Solar Cells Based on Inks of N-Type Colloidal Quantum Dots. ACS Nano 2014, 8 (10), 10321-10327.

(60) Kirmani, A. R.; Carey, G. H.; Abdelsamie, M.; Yan, B.; Cha, D.; Rollny, L. R.; Cui, X.; Sargent, E. H.; Amassian, A. Effect of Solvent Environment on ColloidalQuantum-Dot Solar-Cell Manufacturability and Performance. Adv. Mater. 2014, 26 (27), 4717-4723. 
(61) Maraghechi, P.; Labelle, A. J.; Kirmani, A. R.; Lan, X.; Adachi, M. M.; Thon, S. M.; Hoogland, S.; Lee, A.; Ning, Z.; Fischer, A.; Amassian, A.; Sargent, E. H. The Donor-Supply Electrode Enhances Performance in Colloidal Quantum Dot Solar Cells. ACS Nano 2013, 7 (7), 6111-6116.

(62) Lan, X.; Bai, J.; Masala, S.; Thon, S. M.; Ren, Y.; Kramer, I. J.; Hoogland, S.; Simchi, A.; Koleilat, G. I.; Paz-Soldan, D.; Ning, Z.; Labelle, A. J.; Kim, J. Y.; Jabbour, G.; Sargent, E. H. Self-Assembled, Nanowire Network Electrodes for Depleted Bulk Heterojunction Solar Cells. Adv. Mater. 2013, 25 (12), 1769-1773.

(63) Ip, A. H.; Thon, S. M.; Hoogland, S.; Voznyy, O.; Zhitomirsky, D.; Debnath, R.; Levina, L.; Rollny, L. R.; Carey, G. H.; Fischer, A.; Kemp, K. W.; Kramer, I. J.; Ning, Z.; Labelle, A. J.; Chou, K. W.; Amassian, A.; Sargent, E. H. Hybrid Passivated Colloidal Quantum Dot Solids. Nat. Nanotechnol. 2012, 7 (9), 577582.

(64) Luther, J. M.; Law, M.; Song, Q.; Perkins, C. L.; Beard, M. C.; Nozik, A. J. Structural, Optical, and Electrical Properties of Self-Assembled Films of PbSe Nanocrystals Treated with 1,2-Ethanedithiol. ACS Nano 2008, 2 (2), 271-280.

Zarghami, M. H.; Liu, Y.; Gibbs, M.; Gebremichael, E.; Webster, C.; Law, M. PType PbSe and PbS Quantum Dot Solids Prepared with Short-Chain Acids and Diacids. ACS Nano 2010, 4 (4), 2475-2485.

(66) Brown, P. R.; Kim, D.; Lunt, R. R.; Zhao, N.; Bawendi, M. G.; Grossman, J. C.; Bulović, V. Energy Level Modification in Lead Sulfide Quantum Dot Thin Films through Ligand Exchange. ACS Nano 2014, 8, 5863-5872. 
(67) Kovalenko, M. V.; Scheele, M.; Talapin, D. V. Colloidal Nanocrystals with Molecular Metal Chalcogenide Surface Ligands. Science 2009, 324 (5933), 14171420.

(68) Kovalenko, M. V.; Bodnarchuk, M. I.; Zaumseil, J.; Lee, J.-S.; Talapin, D. V. Expanding the Chemical Versatility of Colloidal Nanocrystals Capped with Molecular Metal Chalcogenide Ligands. J. Am. Chem. Soc. 2010, 132 (29), $10085-10092$.

(69) Jeong, K. S.; Tang, J.; Liu, H.; Kim, J.; Schaefer, A. W.; Kemp, K.; Levina, L.; Wang, X.; Hoogland, S.; Debnath, R.; Brzozowski, L.; Sargent, E. H.; Asbury, J. B. Enhanced Mobility-Lifetime Products in PbS Colloidal Quantum Dot Photovoltaics. ACS Nano 2011, 6 (1), 89-99.

(70) Kim, T.; Gao, Y.; Hu, H.; Yan, B.; Ning, Z.; Jagadamma, L. K.; Zhao, K.;

Kirmani, A. R.; Eid, J.; Adachi, M. M.; Sargent, E. H.; Beaujuge, P. M.; Amassian, A. Hybrid Tandem Solar Cells with Depleted-Heterojunction Quantum Dot and Polymer Bulk Heterojunction Subcells. Nano Energy 2015, 17, 196-205.

(71) Yuan, M.; Kemp, K. W.; Thon, S. M.; Kim, J. Y.; Chou, K. W.; Amassian, A.; Sargent, E. H. High-Performance Quantum-Dot Solids via Elemental Sulfur Synthesis. Adv. Mater. 2014, 26 (21), 3513-3519.

(72) Kim, G.-H.; Walker, B.; Kim, H.-B.; Kim, J. Y.; Sargent, E. H.; Park, J.; Kim, J. Y. Inverted Colloidal Quantum Dot Solar Cells. Adv. Mater. 2014, 26 (20), 33213327.

(73) Kramer, I. J.; Zhitomirsky, D.; Bass, J. D.; Rice, P. M.; Topuria, T.; Krupp, L.; 
Thon, S. M.; Ip, A. H.; Debnath, R.; Kim, H.-C.; Sargent, E. H. Ordered Nanopillar Structured Electrodes for Depleted Bulk Heterojunction Colloidal Quantum Dot Solar Cells. Adv. Mater. 2012, 24 (17), 2315-2319.

(74) Barkhouse, D. A. R.; Debnath, R.; Kramer, I. J.; Zhitomirsky, D.; PattantyusAbraham, A. G.; Levina, L.; Etgar, L.; Grätzel, M.; Sargent, E. H. Depleted Bulk Heterojunction Colloidal Quantum Dot Photovoltaics. Adv. Mater. 2011, 23 (28), $3134-3138$.

(75) Lai, L.-H.; Protesescu, L.; Kovalenko, M. V.; Loi, M. a. Sensitized Solar Cells with Colloidal PbS-CdS Core-shell Quantum Dots. Phys. Chem. Chem. Phys. 2014, 16 (2), 736-742.

(76) Pernik, D. R.; Tvrdy, K.; Radich, J. G.; Kamat, P. V. Tracking the Adsorption and Electron Injection Rates of CdSe Quantum Dots on TiO2 : Linked versus Direct Attachment. J. Phys. Chem. C 2011, 115 (27), 13511-13519.

(77) Carey, G. H.; Kramer, I. J.; Kanjanaboos, P.; Moreno-Bautista, G.; Voznyy, O.; Rollny, L.; Tang, J. A.; Hoogland, S.; Sargent, E. H. Electronically Active Impurities in Colloidal Quantum Dot Solids. ACS Nano 2014, 8 (11), 1176311769.

(78) Weidman, M. C.; Yager, K. G.; Tisdale, W. A. Interparticle Spacing and Structural Ordering in Superlattice PbS Nanocrystal Solids Undergoing Ligand Exchange. Chem. Mater. 2015, 27 (2), 474-482.

(79) Kagan, C. R.; Lifshitz, E.; Sargent, E. H.; Talapin, D. V. Building Devices from Colloidal Quantum Dots. Science (80-. ). 2016, 353 (6302). 
(80) Yang, J.; Choi, M. K.; Kim, D. H.; Hyeon, T. Designed Assembly and Integration of Colloidal Nanocrystals for Device Applications. Adv. Mater. 2016, 28 (6), $1176-1207$.

(81) Zabet-Khosousi, A.; Dhirani, A.-A. Charge Transport in Nanoparticle Assemblies. Chem. Rev. 2008, 108 (10), 4072-4124.

(82) Fischer, A.; Rollny, L.; Pan, J.; Carey, G. H.; Thon, S. M.; Hoogland, S.; Voznyy, O.; Zhitomirsky, D.; Kim, J. Y.; Bakr, O. M.; Sargent, E. H. Directly Deposited Quantum Dot Solids Using a Colloidally Stable Nanoparticle Ink. Adv. Mater. 2013, 25 (40), 5742-5749.

(83) Doris, S. E.; Lynch, J. J.; Li, C.; Wills, A. W.; Urban, J. J.; Helms, B. a. Mechanistic Insight into the Formation of Cationic Naked Nanocrystals Generated under Equilibrium Control. J. Am. Chem. Soc. 2014, 136 (44), 15702-15710.

(84) Gur, I. Air-Stable All-Inorganic Nanocrystal Solar Cells Processed from Solution. Science (80-. ). 2005, 310 (5747), 462-465.

(85) Puthussery, J.; Seefeld, S.; Berry, N.; Gibbs, M.; Law, M. Colloidal Iron Pyrite (FeS 2 ) Nanocrystal Inks for Thin-Film Photovoltaics. J. Am. Chem. Soc. 2011, $133(4), 716-719$.

(86) Giansante, C.; Carbone, L.; Giannini, C.; Altamura, D.; Ameer, Z.; Maruccio, G.; Loiudice, A.; Belviso, M. R.; Cozzoli, P. D.; Rizzo, A.; Gigli, G. Surface Chemistry of Arenethiolate-Capped PbS Quantum Dots and Application as Colloidally Stable Photovoltaic Ink. Thin Solid Films 2014, 560, $2-9$.

(87) Giansante, C.; Carbone, L.; Giannini, C.; Altamura, D.; Ameer, Z.; Maruccio, G.; 
Loiudice, A.; Belviso, M. R.; Cozzoli, P. D.; Rizzo, A.; Gigli, G. Colloidal Arenethiolate-Capped PbS Quantum Dots: Optoelectronic Properties, SelfAssembly, and Application in Solution-Cast Photovoltaics. J. Phys. Chem. C 2013, $117(25), 13305-13317$.

(88) Crisp, R. W.; Panthani, M. G.; Rance, W. L.; Duenow, J. N.; Parilla, P. A.;

Callahan, R.; Dabney, M. S.; Berry, J. J.; Talapin, D. V.; Luther, J. M. Nanocrystal Grain Growth and Device Architectures for High-Efficiency CdTe Ink-Based Photovoltaics. ACS Nano 2014, 8 (9), 9063-9072.

(89) Shooshtari, L.; Rahman, M.; Tajabadi, F.; Taghavinia, N. TiO2 Fibers Enhance Film Integrity and Photovoltaic Performance for Electrophoretically Deposited Dye Solar Cell Photoanodes. ACS Appl. Mater. Interfaces 2011, 3 (3), 638-641.

(90) Boles, M. A.; Ling, D.; Hyeon, T.; Talapin, D. V. The Surface Science of Nanocrystals. Nat. Mater. 2016, 15 (2), 141-153.

(91) Murray, C. B.; Kagan, C. R.; Bawendi, M. G. Self-Organization of CdSe Nanocrystallites into Three-Dimensional Quantum Dot Superlattices. Science (80-. ). 1995, 270 (5240), 1335-1338.

(92) Boles, M. a.; Talapin, D. V. Many-Body Effects in Nanocrystal Superlattices: Departure from Sphere Packing Explains Stability of Binary Phases. J. Am. Chem. Soc. 2015, 137 (13), 4494-4502.

(93) Choi, J. J.; Bealing, C. R.; Bian, K.; Hughes, K. J.; Zhang, W.; Smilgies, D.-M.; Hennig, R. G.; Engstrom, J. R.; Hanrath, T. Controlling Nanocrystal Superlattice Symmetry and Shape-Anisotropic Interactions through Variable Ligand Surface 
Coverage. J. Am. Chem. Soc. 2011, 133 (9), 3131-3138.

(94) Baranov, A. V.; Ushakova, E. V.; Golubkov, V. V.; Litvin, A. P.; Parfenov, P. S.;

Fedorov, A. V.; Berwick, K. Self-Organization of Colloidal PbS Quantum Dots into Highly Ordered Superlattices. Langmuir 2015, 31 (1), 506-513.

(95) Sambur, J. B.; Novet, T.; Parkinson, B. A. Multiple Exciton Collection in a Sensitized Photovoltaic System. Science 2010, 330 (6000), 63-66.

(96) Yu, Y.; Zhang, K.; Sun, S. Effect of Ligands on the Photoluminescence Properties of Water-Soluble PbS Quantum Dots. J. Mol. Struct. 2013, 1031, 194-200.

(97) Ouma, I. L. a; Mushonga, P.; Madiehe, A. M.; Meyer, M.; Dejene, F. B.; Onani, M. O. Synthesis, Optical and Morphological Characterization of MPA-Capped PbSe Nanocrystals. Phys. B Condens. Matter 2014, 439, 130-132.

(98) Dai, Q.; Chen, J.; Lu, L.; Tang, J.; Wang, W. PbS Quantum Dots Prepared by Pulsed Laser Deposition for Photovoltaic Applications and Ligand Effects on Device Performance. Appl. Phys. Lett. 2013, 102 (20), 1-5.

(99) Liang, Y.; Novet, T.; Thorne, J. E.; Parkinson, B. A. Photosensitization of ZnO Single Crystal Electrodes with PbS Quantum Dots. Phys. status solidi 2014, 211 (9), 1954-1959.

(100) Reinhart, C. C.; Johansson, E. Colloidally Prepared 3-Mercaptopropionic Acid Capped Lead Sulfide Quantum Dots. Chem. Mater. 2015, 27 (21), 7313-7320.

(101) Yiannios, C. N.; Karabinos, J. V. Oxidation of Thiols by Dimethyl Sulfoxide. J. Org. Chem. 2002, 28 (11), 3246-3248.

(102) Epstein, W. W.; Sweat, F. W. Dimethyl Sulfoxide Oxidations. Chem. Rev. 1967, 
67 (3), 247-260.

(103) Hens, Z.; Martins, J. C. A Solution NMR Toolbox for Characterizing the Surface Chemistry of Colloidal Nanocrystals. Chem. Mater. 2013, 25 (8), 1211-1221.

(104) Moreels, I.; Fritzinger, B.; Martins, J. C.; Hens, Z. Surface Chemistry of Colloidal PbSe Nanocrystals. J. Am. Chem. Soc. 2008, 130 (45), 15081-15086.

(105) De Roo, J.; Van den Broeck, F.; De Keukeleere, K.; Martins, J. C.; Van Driessche, I.; Hens, Z. Unravelling the Surface Chemistry of Metal Oxide Nanocrystals, the Role of Acids and Bases. J. Am. Chem. Soc. 2014, 136 (27), 9650-9657.

(106) Hassinen, A.; Moreels, I.; de Mello Donegá, C.; Martins, J. C.; Hens, Z. Nuclear Magnetic Resonance Spectroscopy Demonstrating Dynamic Stabilization of CdSe Quantum Dots by Alkylamines. J. Phys. Chem. Lett. 2010, 1 (17), 2577-2581.

(107) Fritzinger, B.; Capek, R. K.; Lambert, K.; Martins, J. C.; Hens, Z. Utilizing SelfExchange to Address the Binding of Carboxylic Acid Ligands to CdSe Quantum Dots. J. Am. Chem. Soc. 2010, 132 (29), 10195-10201.

(108) Sharma, R.; Holland, G. P.; Solomon, V. C.; Zimmermann, H.; Schiffenhaus, S.; Amin, S. A.; Buttry, D. a; Yarger, J. L. NMR Characterization of Ligand Binding and Exchange Dynamics in Triphenylphosphine-Capped Gold Nanoparticles. $J$. Phys. Chem. C 2009, 113 (37), 16387-16393.

(109) Anderson, N. C.; Hendricks, M. P.; Choi, J. J.; Owen, J. S. On the Dynamic Stoichiometry of Metal Chalcogenide Nanocrystals: Spectroscopic Studies of Metal Carboxylate Binding and Displacement. J. Am. Chem. Soc. 2013, 2, $11457-$ 11471. 
(110) Piveteau, L.; Ong, T.-C.; Rossini, A. J.; Emsley, L.; Coperet, C.; Kovalenko, M. V. The Structure of Colloidal Quantum Dots from Dynamic Nuclear Polarization Surface Enhanced NMR Spectroscopy. J. Am. Chem. Soc. 2015, 151016160630009.

(111) Parfenov, P. S.; Litvin, A. P.; Baranov, A. V.; Veniaminov, A. V.; Ushakova, E. V. Calibration of the Spectral Sensitivity of Instruments for the near Infrared Region. J. Appl. Spectrosc. 2011, 78 (3), 433-439.

(112) Young, A. G.; Green, D. P.; McQuillan, A. J. Infrared Spectroscopic Studies of Monothiol Ligand Adsorption on CdS Nanocrystal Films in Aqueous Solutions. Langmuir 2006, 22 (26), 11106-11112.

(113) Young, A. G.; Green, D. P.; McQuillan, A. J. IR Spectroscopic Studies of Adsorption of Dithiol-Containing Ligands on CdS Nanocrystal Films in Aqueous Solutions. Langmuir 2007, 23 (26), 12923-12931.

(114) Owen, J. S. The Coordination Chemistry of Nanocrystal Surfaces. Science (80-. ). 2015, 347 (6222), 615-616.

(115) Hassinen, A.; Moreels, I.; de Mello Donegá, C.; Martins, J. C.; Hens, Z. Supporting Information for Nuclear Magnetic Resonance Spectroscopy Demonstrating Dynamic Stabilization of CdSe Quantum Dots by Alkylamines. $J$. Phys. Chem. Lett. 2010, 1 (17), 2577-2581.

(116) Kuno, M.; Lee, J. K.; Dabbousi, B. O.; Mikulec, F. V.; Bawendi, M. G. The Band Edge Luminescence of Surface Modified CdSe Nanocrystallites: Probing the Luminescing State. J. Chem. Phys. 1997, 106 (23), 9869-9882. 
(117) Terrill, R. H.; Postlethwaite, T. a.; Chen, C.; Poon, C.-D.; Terzis, A.; Chen, A.; Hutchison, J. E.; Clark, M. R.; Wignall, G. Monolayers in Three Dimensions: NMR, SAXS, Thermal, and Electron Hopping Studies of Alkanethiol Stabilized Gold Clusters. J. Am. Chem. Soc. 1995, 117 (50), 12537-12548.

(118) Fritzinger, B.; Moreels, I.; Lommens, P.; Koole, R.; Hens, Z.; Martins, J. C. In Situ Observation of Rapid Ligand Exchange in Colloidal Nanocrystal Suspensions Using Transfer NOE Nuclear Magnetic Resonance Spectroscopy. J. Am. Chem. Soc. 2009, 131 (8), 3024-3032.

(119) Sambur, J. B.; Riha, S. C.; Choi, D.; Parkinson, B. A. Influence of Surface Chemistry on the Binding and Electronic Coupling of CdSe Quantum Dots to Single Crystal TiO 2 Surfaces. Langmuir 2010, 26 (7), 4839-4847.

(120) Baker, D. R.; Kamat, P. V. Tuning the Emission of CdSe Quantum Dots by Controlled Trap Enhancement. Langmuir 2010, 26 (13), 11272-11276.

(121) Chen, X.; Hutchison, J. L.; Dobson, P. J.; Wakefield, G. Highly Luminescent Monodisperse CdSe Nanoparticles Synthesized in Aqueous Solution. J. Mater. Sci. 2009, 44 (1), 285-292.

(122) Kalyuzhny, G.; Murray, R. W. Ligand Effects on Optical Properties of CdSe Nanocrystals. J. Phys. Chem. B 2005, 109 (15), 7012-7021.

(123) Bullen, C.; Mulvaney, P. The Effects of Chemisorption on the Luminescence of CdSe Quantum Dots. Langmuir 2006, 22 (7), 3007-3013.

(124) Nelson, C. a; Zhu, X. Y. Reversible Surface Electronic Traps in PbS Quantum Dot Solids Induced by an Order-Disorder Phase Transition in Capping Molecules. $J$. 
Am. Chem. Soc. 2012, 134 (18), 7592-7595.

(125) Brouwer, A. M. Standards for Photoluminescence Quantum Yield Measurements in Solution (IUPAC Technical Report). Pure Appl. Chem. 2011, 83 (12), 22132228.

(126) Dirin, D. N.; Dreyfuss, S.; Bodnarchuk, M. I.; Nedelcu, G.; Papagiorgis, P.; Itskos, G.; Kovalenko, M. V. Lead Halide Perovskites and Other Metal Halide Complexes as Inorganic Capping Ligands for Colloidal Nanocrystals. J. Am. Chem. Soc. 2014, 136 (18), 6550-6553.

(127) Moody, I. S.; Stonas, A. R.; Lonergan, M. C. PbS Nanocrystals Functionalized with a Short-Chain, Ionic, Dithiol Ligand. J. Phys. Chem. C 2008, 112 (49), $19383-19389$.

(128) Mann, J. R.; Watson, D. F. Adsorption of CdSe Nanoparticles to Thiolated TiO2 Surfaces: Influence of Intralayer Disulfide Formation on CdSe Surface Coverage. Langmuir 2007, 23 (22), 10924-10928.

(129) Aldana, J.; Wang, Y. A.; Peng, X. Photochemical Instability of CdSe Nanocrystals Coated by Hydrophilic Thiols. J. Am. Chem. Soc. 2001, 123 (36), 8844-8850.

(130) Koole, R.; Luigjes, B.; Tachiya, M.; Pool, R.; Vlugt, T. J. H.; de Mello Donegá, C.; Meijerink, A.; Vanmaekelbergh, D. Differences in Cross-Link Chemistry between Rigid and Flexible Dithiol Molecules Revealed by Optical Studies of CdTe Quantum Dots. J. Phys. Chem. C 2007, 111 (30), 11208-11215.

(131) Zhu, H.; Hu, M. Z.; Shao, L.; Yu, K.; Dabestani, R.; Zaman, M. B.; Liao, S. Synthesis and Optical Properties of Thiol Functionalized CdSe/ZnS (Core/Shell) 
Quantum Dots by Ligand Exchange. J. Nanomater. 2014, 2014, 1-14.

(132) Koole, R.; Liljeroth, P.; De Mello Donegá, C.; Vanmaekelbergh, D.; Meijerink, A. Electronic Coupling and Exciton Energy Transfer in CdTe Quantum-Dot Molecules. J. Am. Chem. Soc. 2006, 128 (28), 10436-10441.

(133) Kovalenko, M. V.; Scheele, M.; Talapin, D. V. Colloidal Nanocrystals with Molecular Metal Chalcogenide Surface Ligands. Science (80-. ). 2009, 324 (5933), $1417-1420$.

(134) Nag, A.; Kovalenko, M. V.; Lee, J.-S.; Liu, W.; Spokoyny, B.; Talapin, D. V. Metal-Free Inorganic Ligands for Colloidal Nanocrystals: S(2-), HS(-), Se(2-), $\mathrm{HSe}(-), \mathrm{Te}(2-), \mathrm{HTe}(-), \mathrm{TeS}(3)(2-), \mathrm{OH}(-)$, and $\mathrm{NH}(2)(-)$ as Surface Ligands. $J$. Am. Chem. Soc. 2011, 0-8.

(135) De Roo, J.; Ibáñez, M.; Geiregat, P.; Nedelcu, G.; Walravens, W.; Maes, J.; Martins, J. C.; Van Driessche, I.; Kovalenko, M. V.; Hens, Z. Highly Dynamic Ligand Binding and Light Absorption Coefficient of Cesium Lead Bromide Perovskite Nanocrystals. ACS Nano 2016, 10 (2), 2071-2081.

(136) Wuister, S. F.; de Mello Donegá, C.; Meijerink, A. Influence of Thiol Capping on the Exciton Luminescence and Decay Kinetics of CdTe and CdSe Quantum Dots. J. Phys. Chem. B 2004, 108 (45), 17393-17397.

(137) Kovalenko, M. V.; Bodnarchuk, M. I.; Talapin, D. V. Nanocrystal Superlattices with Thermally Degradable Hybrid Inorganic-Organic Capping Ligands. J. Am. Chem. Soc. 2010, 132 (43), 15124-15126.

(138) Cass, L. C.; Malicki, M.; Weiss, E. A. The Chemical Environments of Oleate 
Species within Samples of Oleate-Coated PbS Quantum Dots. Anal. Chem. 2013, 85 (14), 6974-6979.

(139) Malcolm H. Levitt. Spin Dynamics - Basics of Nuclear Magnetic Resonance, Second Edi.; John Wiley \& Sons, Inc, 2008.

(140) Lan, X.; Voznyy, O.; García de Arquer, F. P.; Liu, M.; Xu, J.; Proppe, A. H.; Walters, G.; Fan, F.; Tan, H.; Liu, M.; Yang, Z.; Hoogland, S.; Sargent, E. H. 10.6\% Certified Colloidal Quantum Dot Solar Cells via Solvent-PolarityEngineered Halide Passivation. Nano Lett. 2016, 16 (7), 4630-4634.

(141) Lan, X.; Voznyy, O.; Kiani, A.; García de Arquer, F. P.; Abbas, A. S.; Kim, G.-H.; Liu, M.; Yang, Z.; Walters, G.; Xu, J.; Yuan, M.; Ning, Z.; Fan, F.; Kanjanaboos, P.; Kramer, I.; Zhitomirsky, D.; Lee, P.; Perelgut, A.; Hoogland, S.; Sargent, E. H. Passivation Using Molecular Halides Increases Quantum Dot Solar Cell Performance. Adv. Mater. 2015, 28 (2), 299-304.

(142) Ning, Z.; Ren, Y.; Hoogland, S.; Voznyy, O.; Levina, L.; Stadler, P.; Lan, X.; Zhitomirsky, D.; Sargent, E. H. All-Inorganic Colloidal Quantum Dot Photovoltaics Employing Solution-Phase Halide Passivation. Adv. Mater. 2012, 24 (47), 6295-6299.

(143) Gomes, R.; Hassinen, A.; Szczygiel, A.; Zhao, Q.; Vantomme, A.; Martins, J. C.; Hens, Z. Binding of Phosphonic Acids to CdSe Quantum Dots: A Solution NMR Study. J. Phys. Chem. Lett. 2011, 2 (3), 145-152.

(144) Suga, K.; Yokoi, T.; Kondo, D.; Hayashi, K.; Morita, S.; Okamoto, Y.; Shimanouchi, T.; Umakoshi, H. Systematical Characterization of Phase Behaviors 
and Membrane Properties of Fatty Acid/didecyldimethylammonium Bromide Vesicles. Langmuir 2014, 30 (43), 12721-12728.

(145) Bricker, J. C.; Laricchia, L. Advances in Merox ${ }^{\mathrm{TM}}$ Process and Catalysis for Thiol Oxidation. Top. Catal. 2012, 55 (19-20), 1315-1323.

(146) Li, X.-B.; Li, Z.-J.; Gao, Y.-J.; Meng, Q.-Y.; Yu, S.; Weiss, R. G.; Tung, C.-H.; Wu, L.-Z. Mechanistic Insights into the Interface-Directed Transformation of Thiols into Disulfides and Molecular Hydrogen by Visible-Light Irradiation of Quantum Dots. Angew. Chemie Int. Ed. 2014, 53 (8), 2085-2089. 